THE BIOPHYSICS OF TITIN IN CARDIAC HEALTH AND DISEASE

by

Brian R. Anderson

A Dissertation Submitted to the Faculty of the

DEPARTMENT OF PHYSICS

In Partial Fulfillment of the Requirements

For the Degree of

DOCTOR OF PHILOSOPHY

In the Graduate College

THE UNIVERSITY OF ARIZONA

2014 


\section{THE UNIVERSITY OF ARIZONA \\ GRADUATE COLLEGE}

As members of the Dissertation Committee, we certify that we have read the dissertation prepared by Brian Anderson, titled The Biophysics of Titin in Cardiac Health and Disease and recommend that it be accepted as fulfilling the dissertation requirement for the Degree of Doctor of Philosophy.

Date: 24 April 2014

Henk Granzier

Date: 24 April 2014

Andrew Hausrath

Date: 24 April 2014

Brian LeRoy

Date: 24 April 2014

Srin Manne

Date: 24 April 2014

Koen Visscher

Final approval and acceptance of this dissertation is contingent upon the candidate's submission of the final copies of the dissertation to the Graduate College.

I hereby certify that I have read this dissertation prepared under my direction and recommend that it be accepted as fulfilling the dissertation requirement.

Date: 24 April 2014

Dissertation Director: Henk Granzier

Date: 24 April 2014

Dissertation Director: Koen Visscher 


\section{STATEMENT BY AUTHOR}

This dissertation has been submitted in partial fulfillment of the requirements for an advanced degree at the University of Arizona and is deposited in the University Library to be made available to borrowers under rules of the Library.

Brief quotations from this dissertation are allowable without special permission, provided that an accurate acknowledgement of the source is made. Requests for permission for extended quotation from or reproduction of this manuscript in whole or in part may be granted by the head of the major department or the Dean of the Graduate College when in his or her judgment the proposed use of the material is in the interests of scholarship. In all other instances, however, permission must be obtained from the author.

SIGNED: Brian R. Anderson 
List of Figures and Tables...........................................................

Abstract..................................................................6

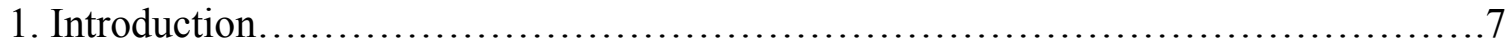

2. AFM................................................................... 9

3. Serially-Linked Springs............................................... 17

4. Bell's Formula and Monte Carlo Simulations..................................19

5. Beyond Single Molecules...............................................23

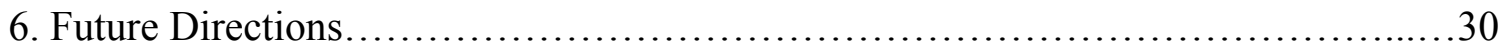

7. Investigating the link between the T16I Ig10 mutation and arrhythmogenic cardiomyopathy using a knock-in (KI) mouse model (manuscript in progress).........35

References............................................................53

Appendix A-Single Molecule Force Spectroscopy on Titin Implicates Immunoglobulin Domain Stability as a Cardiac Disease Mechanism,

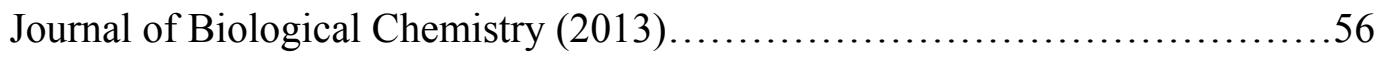

Appendix B-Titin-Based Tension in the Cardiac Sarcomere: Molecular Origin and Physiological Adaptations, Progress in Biophysics and

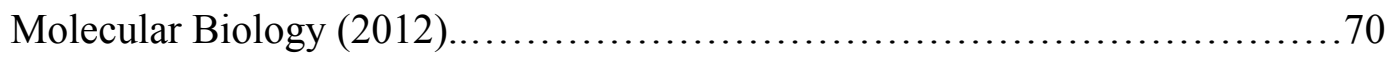
Appendix C-The Effects of PKC $\alpha$ Phosphorylation of the Extensibility of Titin's PEVK Element, Journal of Structural Biology (2010).................85 


\section{List of Figures and Tables}

Table 1: Ig10 unfolding forces determined by AFM...............................14

Figure 1: Gaussian fits to Ig10 unfolding force distributions........................15

Figure 2: Corrected and uncorrected Ig10 distributions.......................... 16

Figure 3: CDFs of unfolding forces and collapse onto parent CDF.................... 16

Table 2: Effect of $\alpha_{0}$ and $\Delta \mathrm{x}$ on standard deviation of unfolding forces...............21

Table 3: Unfolding rates as function of clamp force.............................22

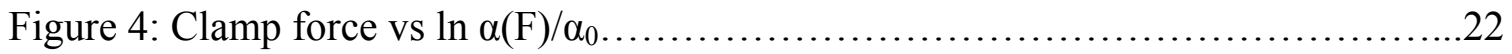

Figure 5: Targeting vector strategy for AC mouse model...........................24

Figure 6: Echo parameters............................................... 27

Figure 7: PSR staining of cardiac cross sections.....................................

Figure 8: Oil red $\mathrm{O}$ staining of cardiac cross sections.............................29

Figure 9: Quantification of titin isoforms..................................... 30

Figure10: Unfolding rate and pulling speed independence......................... 31

Figure A1: Sarcomere schematic and Ig10 location...............................

Figure A2: Ig10 Western Blots...........................................43

Figure A3: Titin isoforms and titin degradation...............................44

Figure A4: Echo parameters.................................................46

Figure A5: PSR staining of cardiac cross sections............................47

Figure A6: Oil red O staining of cardiac cross sections $\ldots \ldots \ldots \ldots \ldots \ldots \ldots \ldots \ldots \ldots \ldots . \ldots 48$

Figure A7: Passive tension of skinned muscle strips............................49

Figure A8: Viscous stress of skinned muscle strips............................50

Figure A9: Elastic and viscous moduli of skinned muscle strips....................50 


\begin{abstract}
The giant protein titin is the third myofilament in the cardiac sarcomere. It is responsible for generating passive forces in stretched myocardium and maintaining sarcomere structure. The force generation properties of titin are determined by titin's elastic springlike elements, and this dissertation focuses on the determination of the physical properties of these springlike elements using atomic force microscopy. The primary project of this dissertation investigates the link between a single point mutation in one of titin's subdomains and arrhythmogenic cardiomyopathy.
\end{abstract}




\section{Introduction}

The cardiac sarcomere contains three large myofilaments that account for force generation in the beating heart. While interaction between the actin-based thin filament and myosin-based thick filament is responsible for active force generation (contraction), the giant protein titin helps determine passive force levels in the sarcomere. A single titin molecule spans half the sarcomere and connects the thin and thick filaments (Appendix A Figure 1). In between the filament-binding regions of titin is titin's elastic I-band, which is thought to be mostly unbound in the sarcomere and is the region of titin that is primarily responsible for generation of titin-based passive forces. Although the extracellular matrix also contributes to passive forces in stretched myocardium, titin is responsible for the majority of passive force within the physiological sarcomere length range.

The I-band region of titin consists of three serially-linked (titin is a single continuous polypeptide) segments that have distinct physical characteristics: tandem immunoglobulin(Ig)-like domains, the PEVK element, and the N2B element. These segments behave like a molecular spring, and the forces they generate upon sarcomere stretch are important for overall sarcomere structure. For example, because each half of the sarcomere contains an equal number of titin molecules (symmetry considerations dictate this fact), the thick filaments of the sarcomere experience equal and opposite titinbased passive force upon sarcomere stretch, which maintains the thick filaments in the center of the sarcomere during diastole [1]. It has been shown previously that degradation of titin leads to unbalanced passive forces in the sarcomere and thick filament displacement from the center of the sarcomere towards either of the protein-rich Z-disks 
upon active contraction [2]. Most importantly, this loss of titin function prevents recentering of the thick filament upon myofilament relaxation and critically damages sarcomeres. This example is one of many that underscore the fact that titin-based passive forces are an integral part of proper cardiac function. Diastolic function - the ability of the ventricles to relax and receive blood from the atria - is largely determined by titinbased passive stiffness, and changes in the physical properties of titin's elastic region are associated with diastolic dysfunction and cardiac disease. Titin-based passive tension is altered by two primary mechanisms: titin isoform splicing and post-translational modifications.

There are two titin isoforms in the adult heart (Appendix B Figure 1) that are largely identical except for their elastic I-band regions. The N2B isoform has a shorter Iband region than the N2BA isoform and therefore is stiffer (i.e. requires more force to stretch and, concurrently, develops a higher restoring force). Changes in titin isoform expression have been associated with disease states, including a dilated cardiomyopathy dog model [3] and a hypertensive rat model [4]. In humans, patients with coronary artery disease express a higher percentage of N2BA isoforms that corresponds with decreased stiffness at the myofibril level [5], and patients with dilated cardiomyopathy have also shown a higher N2BA:N2B ratio and lower passive stiffness.

Various protein kinases preferentially phosphorylate specific elastic segments of titin's I-band that lead to changes in titin stiffness at the single molecule and tissue level [6-7]. For example, protein kinases A and G (PKA and PKG) phosphorylate the N2B element of titin, while PKC phosphorylates the PEVK element. Phosphorylation of titin can lead to increased [7] or decreased [6] passive stiffness, depending on the specific site 
of phosphorylation. The effects of site specific phosphorylation can be directly measured with single-molecule techniques, and information garnered from such experiments can be very useful for suggesting therapies to combat cardiac dysfunction related to changes in myocardial stiffness. For example, molecules that alter $\alpha$ - and $\beta$-adrenergic stimulation (which activate downstream kinases) can be used to modify PKA/PKC activity which will then tune titin-based stiffness levels.

The three elastic segments of titin have distinct mechanical properties under baseline conditions and respond differently to phosphorylation and mutation in a sitespecific manner. For this reason it is necessary to study these segments individually. A powerful method for studying the physical properties of proteins at the single molecule level is atomic force microscopy (AFM), which directly measures the force needed to stretch a protein. While powerful, care must be taken to design and analyze AFM experiments to avoid erroneous results and establish confidence in the technique. In particular, two aspects must be considered: the design of recombinant protein fragments and analysis of AFM data.

\section{AFM}

All of my studies were performed with a MFP3D AFM (Asylum Research, Santa Barbara, CA). Purified recombinant protein in buffer $(\sim 5-10 \mathrm{ug} / \mathrm{mL})$ was spotted on a clean microscope slide and incubated overnight at $4^{\circ} \mathrm{C}$. Protein density was kept low to minimize protein-protein interactions and the chance than multiple protein molecules would attach to the cantilever tip simultaneously. Both of these scenarios can be detected by irregular force-extension traces that are inconsistent with the molecular fingerprint of 
the protein of interest. Proteins randomly and nonspecifically attach to the piezo-driven silicon nitride cantilever tip (MLCT; Bruker) as the tip contacts the protein-coated slide surface. If a protein sticks to the cantilever, a force-extension trace is recorded as the tip retracts from the slide surface until the protein is displaced from either the slide surface or cantilever tip. The displacement of the cantilever base is directly measured by an integrated linear voltage differential transformer. Bending of the cantilever tip is measured by using an optical lever and quadrant photodetector system that is then converted to force by using Hooke's Law $F=-k x$. Cantilever stiffness is determined by using the equipartition theorem. For more information see Materials and Methods in Appendix A or C.

\section{Synthetic Protein Design}

When stretching a protein with AFM, the only output the user sees is the force (converted from tip displacement) and the extension (converted from cantilever base position) generated by whatever object was attached to the cantilever tip upon cantilever retraction. All parameters describing the tethered protein must be inferred from this force vs extension trace. Therefore, the protein of interest being stretched needs to be in solution absent other protein contaminants, and some knowledge about the protein's structure is preferred. If various proteins are present during an AFM experiment, it cannot be known with certainty which protein was stretched that resulted in the force vs extension output. Knowing the size and generic structure of a protein helps, but this information is not always sufficient. Even when a protein solution is pure, it is not guaranteed that a single protein is being stretched; for example, it is common to stretch 
two protein molecules simultaneously or to stretch proteins that are interacting with each other (such as dimers). In order to have confidence that a single protein is being stretched, the protein should have a characteristic pattern when stretched and/or unfolded (such as the five equally-spaced, low force peaks in Appendix A Figure 2). This molecular fingerprint is a good indicator of when a single protein is tethered and stretched using AFM. Proteins lacking well-defined structure, such as the PEVK element of titin, do not generate a clear fingerprint when stretched - they lack large structural transitions upon stretch that would result in identifiable force peaks. Therefore, additional protein domains are often inserted next to the protein of interest to generate a fingerprint. This idea was used in my AFM study on the PEVK element of titin (Appendix C). As its name suggests, the PEVK element contains a high concentration of proline $(\mathrm{P})$, glutamate (E), valine $(\mathrm{V})$, and lysine $(\mathrm{K})$ residues. Although NMR and circular dichroism experiments suggest polyproline helix-coil motifs are present in the PEVK [8-9], its high proline concentration hinders the formation of large $\alpha$-helix and $\beta$-sheet structures [10]. Most of the large force peaks recorded by AFM are due to large tertiary structure transitions, which the PEVK element lacks. Therefore, the PEVK element is often expressed in combination with titin Ig domains. Ig domains form a well-described $\beta$ sandwich structure that results in large unfolding force peaks upon stretch. The contour length gain following Ig domain unfolding (as determined by fitting the AFM data with the wormlike chain equation [11]) is known by the length difference between a folded Ig domain (4-5 nm) and an unfolded, random coil Ig domain (number of residues $\times 0.38$ $\mathrm{nm} /$ residue). Linking Ig domains to intrinsically disordered proteins like PEVK imparts a molecular fingerprint on the PEVK-Ig fragment for AFM study. 
I also studied the mechanics and kinetics of Ig domains using AFM. In particular, I investigated how a disease-linked point mutation affects Ig 10 (the $10^{\text {th }} \operatorname{Ig}$ domain of titin's proximal Ig segment—Appendix A Figure 1). For this project I designed Ig10 pentamers-five identical Ig10 domains connected in series (synthetic DNA sequences coding for these fragments were purchased from Geneart (Regensburg, Germany)). Although this design is not native (Ig10 is flanked by $\operatorname{Ig} 9$ and $\operatorname{Ig} 11$ natively), it is necessary in order to guarantee that each unfolding peak in the molecular fingerprint is due to Ig10 unfolding. For example, if I stretched and unfolded an Ig9-Ig10-Ig11 fragment, a three peak force-extension trace would be measured, but it would be impossible to assign a given peak to a certain domain's unfolding. With an Ig10 repeat fragment, each unfolding peak is due to Ig10. The main concern in designing the $\operatorname{Ig} 10$ pentamer is to maintain the native rotational constraints between adjacent Ig10 domains. This is done by choosing the short amino acid linker sequences between each Ig10 domain such that the amino acids flanking Ig10 are the same as is present natively. Because at the time the atomic structure of Ig10 was unknown, I used secondary structure prediction (Jpred [12]) and multiple sequence alignment (ClustalW [13]) algorithms to determine the interfaces between the globular domain of Ig10 and the short linker sequences that connect it to adjacent domains. I also included two cysteine residues at the C-terminal of the pentamers to allow for thiol-gold bonds to anchor the protein to the gold-coated microscope slide. Few attachment chemistries allow for strong binding to gold, although the thiol group binds to gold with a bond strength of $\sim 44 \mathrm{kcal} / \mathrm{mol}$ [14]. This promotes C-terminal binding to the microscope slide while minimizing attachment points between the globular Ig domains and the slide. 
Analysis

The force needed to stretch an elastic biomolecule is well-described by the wormlike chain (WLC) equation [11]: $F=\frac{k_{B} T}{L_{p}}\left(\frac{z}{L_{c}}+\frac{1}{4\left(1-z / L_{c}\right)^{2}}-\frac{1}{4}\right)$. This equation is an approximate interpolation formula that describes the force needed to extend a polymer. The extension (end-to-end length) of the molecule $(z)$ divided by its contour length $\left(\mathrm{L}_{\mathrm{c}}\right)$ is referred to as the fractional extension, with the force needed to extend the molecule rapidly increasing as $\mathrm{z} / \mathrm{L}_{\mathrm{c}}$ approaches 1 . The persistence length of the molecule $\left(\mathrm{L}_{\mathrm{p}}\right)$ is a measure of its bending rigidity and is inversely proportional to the force needed to extend the molecule. It is counterintuitive that a more flexible protein (lower $\mathrm{L}_{\mathrm{p}}$ ) requires more external force to stretch, because this is the same thing as saying that a more flexible protein is a stiffer spring than a less flexible protein. This behavior is explained by realizing that random coil proteins (such as the PEVK element of titin) behave as entropic springs, not enthalpic springs (although at high fractional extensions bond stretching must be considered). See Section 3 of Appendix B for a qualitative discussion on the matter.

The persistence length and contour length of an elastic protein can be estimated by fitting the force-extension trace with the WLC equation. When an elastic, predominantly unstructured protein is connected in series with a more stable domain, the force-extension trace leading up to the first unfolding peak describes the behavior of the elastic region of the protein fragment (Appendix C Figure 1). In my PEVK study, I looked at the effect that point mutations and phosphorylation had on the persistence length and contour length of PEVK by analyzing the force-extension trace up to the first Ig unfolding event with the WLC equation. I found that phosphorylation of two serine 
residues in the PEVK element reduces the persistence length of PEVK (consistent with increased titin-based passive tension at the tissue level [7]) and that mutation of these residues to alanine similarly reduced PEVK persistence length (see Appendix C for details).

My main research project has investigated a disease-linked point mutation (T16I) found in Ig10. The link between this titin mutation and the specific cardiac disease (arrhythmogenic cardiomyopathy; see Beyond Single Molecules for discussion) is still being investigated, but the critical first step was to determine if the mutation has an effect at the single molecule level. I performed three different AFM stretch protocols (stretch, refolding, and force clamp) on recombinant Ig10 pentamers (five native $\operatorname{Ig} 10$ domains connected in series (WT Ig10 5mer) or five mutant $\operatorname{Ig} 10$ domains connected in series (T16I Ig10 5mer)). The fragments exhibit a well-defined molecular fingerprint of five unfolding force peaks separated by $\sim 28 \mathrm{~nm}$ periodicity (see Appendix A Figure 2). Although each unfolding force peak is attributed to Ig10, the average unfolding force increases as a function of peak number (i.e. the unfolding force of peak 1 is the lowest on average and the unfolding force of peak 5 is the highest; Table 1).

Table 1: Average unfolding force $(\mathrm{pN})$ of WT and T16I Ig10 pentamers

\begin{tabular}{|c|c|c|c|c|c|}
\hline & Peak1 & Peak 2 & Peak 3 & Peak 4 & Peak 5 \\
\cline { 1 - 5 } WT & $120 \pm 2$ & $124 \pm 2$ & $128 \pm 2$ & $134 \pm 2$ & $143 \pm 4$ \\
\cline { 1 - 5 } T16I & $92 \pm 2 * * *$ & $97 \pm 3 * * *$ & $106 \pm 2 * * *$ & $117 \pm 2 * * *$ & $126 \pm 4 * *$ \\
$* * * \mathrm{p}<0.001$
\end{tabular}

Initial AFM work on heteropolyprotein Ig fragments reasoned that the peak dependence of unfolding force indicates that less stable Ig domains unfold before more stable Ig domains [15-16]. However, this peak dependence on unfolding force was also seen when homopolyproteins were stretched [17]. Because each unfolding force peak was 
due to identical domains unfolding, these studies analyzed unfolding force data with all unfolding force peaks combined (as is shown in Figure 1 for my $\operatorname{Ig} 10$ pentamer data). This results in unfolding force distributions that have a lower mean compared to the distribution describing the unfolding probability of a single (isolated) domain (Figure 2). The peak dependence of unfolding force is due to the combinatorics of the system (see section 5.4.1 of Appendix B for more information), but a theoretical method based on order statistics theory was developed [18] that removed the peak dependence of unfolding forces and allowed for all unfolding force data to collapse onto a single distribution (describing the unfolding force of a single isolated domain; Figure 3). This allows all unfolding peaks to be analyzed together, which quintuples (in the case of homopolyprotein pentamers) the unfolding force data set and results in more accurate fitting procedures. My work was the first to show that this theory can be effectively applied to experimental data (Appendix A).
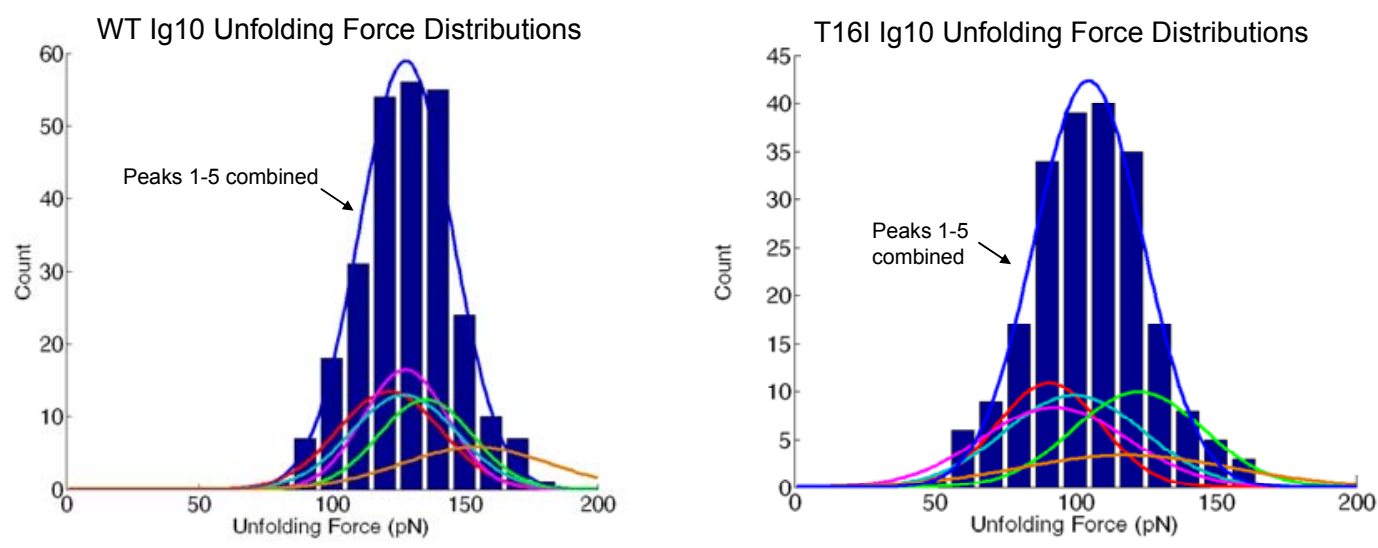

Figure 1: Gaussian fits to the distribution of each unfolding force peak from WT and T16I Ig10 pentamers. The five small distributions are for peaks 1-5 and the large distribution (blue line and histogram) are with all unfolding force peaks combined. Red $=$ peak 1 , magenta $=$ peak 2 , cyan $=$ peak 3 , green $=$ peak 4 , orange $=$ peak 5 . 

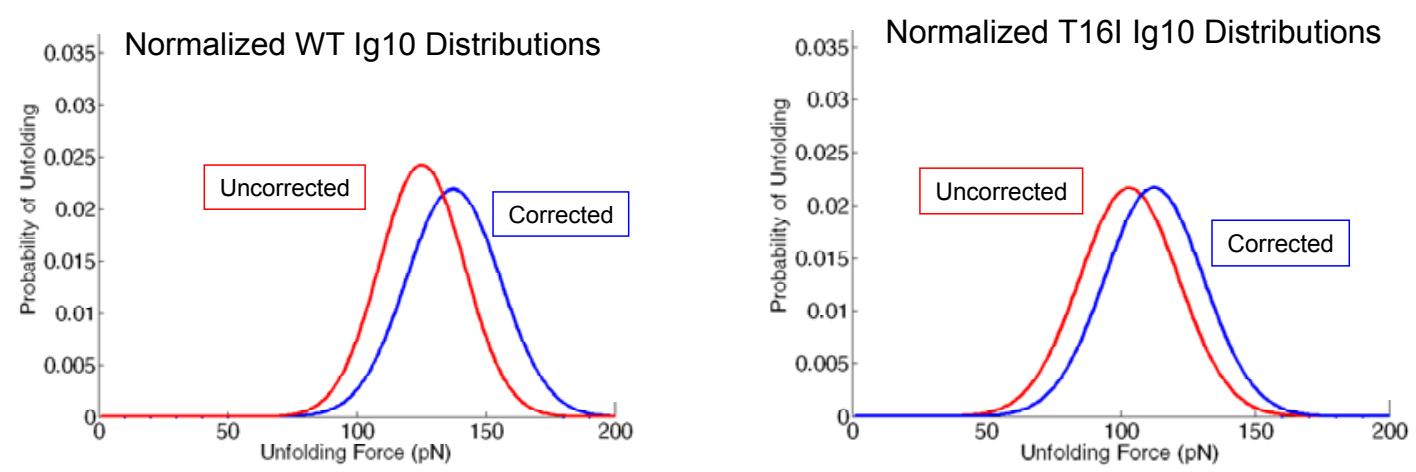

Figure 2: The red lines represent the unfolding force distributions of Ig10 pentamers when the peak dependence of unfolding force is not corrected for (same as blue Gaussian lines in Figure 1). The blue lines are the unfolding force distributions that describe a single isolated domain (i.e. after correcting for the peak dependence of unfolding force). Notice that combining all unfolding force peaks underestimates the actual mean unfolding force. The corrected distributions are determined by using order statistics theory; see Figure 3.
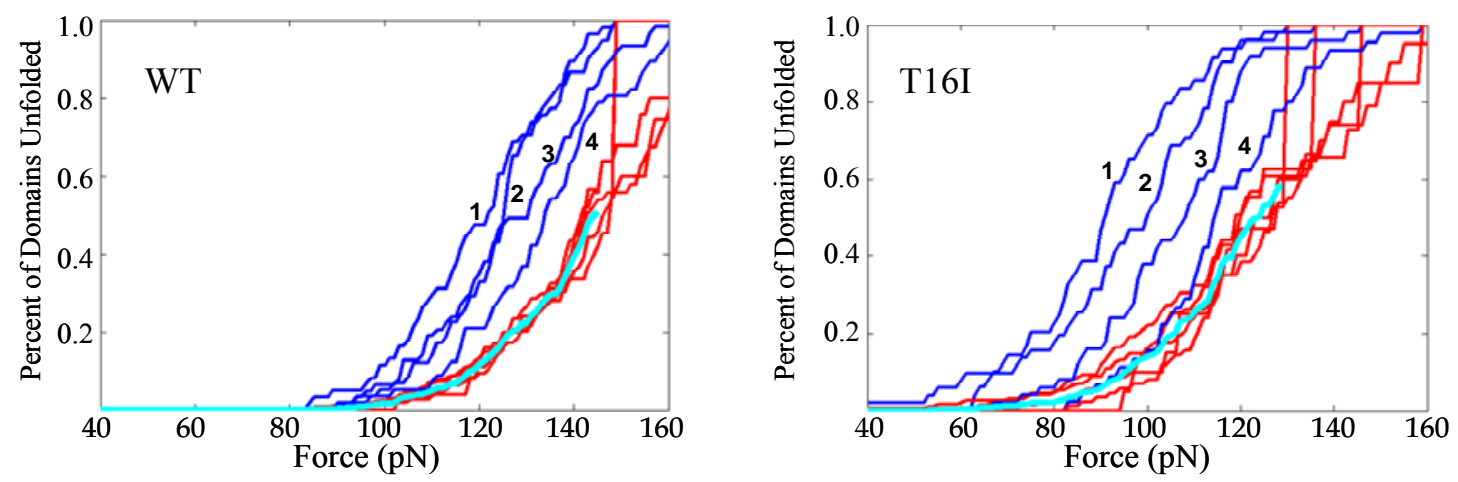

Figure 3: Cumulative distribution functions of the unfolding forces of each peak in WT (left) and T16I (right) Ig10 pentamers. The blue traces are the uncorrected cdfs of peaks 1-4, while the red peaks are the corrected cdfs after correcting for order statistics. Notice that the transformation collapses the cdfs onto a master curve describing a single domain (peak 5 of the unfolded Ig10 pentamer, by definition, represents the unfolding force distribution of a single domain). The average of these transformed cdfs is used to determine the "corrected" unfolding force distribution shown in Figure 2.

Although not directly measured, the unfolding rate of a protein $\left(\alpha_{0}\right)$ is an important parameter that can be determined from unfolding force distributions. In the case of Ig domains of titin, unfolding rate is important because the average unfolding force of an Ig domain under AFM pulling velocities is greater than $100 \mathrm{pN}$ [19], which is over 20 times more than the tension present in titin's elastic I-band region at the high end of the physiological sarcomere length range [20]. Since the unfolding rate of a protein 
describes the rate under zero net external force that a protein transitions from its native (folded) state to an unfolded state due to stochastic fluctuations, if we know $\alpha_{0}$ for a protein we have a much better idea of its propensity to unfold under physiological force levels $(<5 \mathrm{pN})$ compared to only knowing its behavior at high force levels (such as in AFM pulling experiments). Force-dependent unfolding is often assumed to follow Bell's formula [21]: $\alpha(F)=\alpha_{0} \cdot \exp \left(F \cdot \Delta x / k_{B} T\right)$, where $F$ is the force acting on the molecule and $\Delta x$ is the distance along the reaction coordinate from the folded state to the transition barrier. This formula was introduced to describe how force accelerates the disruption of chemical bonds and is clearly a simplification when used in the context of protein unfolding. However, Bell's formula does fit my experimental data surprisingly well (see Bell's Formula and Monte Carlo Simulations). Since I can infer $\alpha_{0}$ from my experimental data and use Bell's formula to scale the unfolding rate to determine $\alpha(F)$ at intracellular force levels, I can estimate the rate of protein unfolding under physiological conditions (see Figure 9 Appendix A).

\section{Serially-Linked Springs}

Although it is important to study the springlike elements of titin's I-band region individually, knowing the mechanical properties of one of the elements does not explain how the three elements work in series. Because the entire I-band region of titin experiences the same tension at any time, the mechanical properties of the tandem Ig domains, PEVK element, and N2B element need to be known in order to determine their collective contribution to passive tension as a function of sarcomere length. It has been shown that the three elastic elements extend to different degrees depending on the 
sarcomere length [22], with the tandem Ig domains straightening out (but not unfolding) prior to elongation of the end-to-end distance of the PEVK and N2B elements. When the sarcomere is stretched, the most compliant regions of titin's I-band are the first to extend which results in a low titin-based restoring force. As sarcomere length increases, the less compliant regions of titin are extended, which necessarily corresponds with a higher titinbased restoring force. This passive interplay within titin's elastic region can also be seen in the fact that mouse models missing one element of titin's I-band region show an increased, compensatory extension in the remaining springlike elements [23].

Since the contour length and persistence length of the tandem Ig domains, PEVK element, and N2B element are known from their primary sequences and single molecule studies, I can determine the force-extension characteristic of the entire I-band region of titin. To do this, an inverted WLC model is needed that describes the extension of a polymer as function of force, contour length, and persistence length. A simple formula for this is such [24]: $z\left(F, L_{c}, L_{p}\right)=L_{c}\left[1-\frac{1}{2}\left(\frac{L_{p} F}{k_{B} T}-\frac{1}{32}\right)^{-1 / 2}\right]$. With this equation, I first determine $z\left(F, L_{c}, L_{p}\right)$ for each springlike element for all the force values of interest. Then, assuming force balance across the three elastic segments, I sum the $z\left(F, L_{c}, L_{p}\right)$ values for the tandem Ig segment, the PEVK element, and the N2B element to generate total extension as a function of force. With this method, results from single molecule studies can be translated to the tissue level. For example, if phosphorylation of the PEVK element changes its persistence length, the effect of this phosphorylation on the overall force-extension behavior of titin's I-band region can be estimated. This allows for comparison between data at the single molecule level and at the tissue level, the latter of 
which is determined by the entire I-band region of full-length titin molecules that are packed into sarcomeres at an approximate density of 3 billion titin molecules per $\mathrm{mm}^{2}$ [25]. A major benefit of this extrapolation from single molecule studies to tissue level is that it helps determine if effects seen at the tissue level are due entirely from primary changes at the single molecule level. For instance, if protein kinase activity increases passive tension in skinned (demembranated) cardiac tissue, it cannot be known if this effect is due solely to titin phosphorylation, since most kinases have a variety of sarcomeric substrates. However, if this passive tension increase is fully explained by a change in the physical properties of an elastic element of titin (by using single molecule studies in concert with the inverted WLC model), then it can be concluded that a particular elastic element (or even specific residue) of titin is responsible for the passive tension changes seen at the tissue level following phosphorylation treatment.

\section{Bell's Formula and Monte Carlo Simulations}

The force dependence of Ig domain unfolding is often assumed to follow Bell's formula [21]: $\alpha(F)=\alpha_{0} \cdot \exp \left(F \cdot \Delta x / k_{B} T\right)[17,20,26]$. This formula has been assumed because of its simplicity which allows it to be easily integrated into Monte Carlo (MC) simulations of Ig domain unfolding. MC simulations are used to infer $\alpha_{0}$ and $\Delta x$, the unfolding rate of a protein and the distance from the folded state to the transition barrier (which separates the folded and unfolded states) along some reaction coordinate, respectively. The purpose of MC simulations in regards to Ig domain unfolding is to generate large unfolding force (UF) distributions that depend on two parameters $\left(\alpha_{0}, \Delta x\right)$. These distributions are then compared to experimental UF distributions to find the $\left(\alpha_{0}\right.$, 
$\Delta x)$ parameter pair that best reproduces the experimental data. This is how the unfolding rate of an Ig domain under no external force can be estimated from UF distributions that are centered between $100-200 \mathrm{pN}$.

In my MC simulations, experimental conditions were mimicked as best as possible. I start with 5 folded Ig domains of known diameter and stretch the entire tandem at $1000 \mathrm{~nm} / \mathrm{s}$ (AFM pulling velocity) by increasing their end-to-end length in $40 \mu \mathrm{s}(0.4$ A) intervals. At each interval, the force acting on the Ig tandem is determined by force balance between cantilever bend $(|F|=k x)$ and the force required to stretch the polypeptide using the wormlike chain equation. The probability of an Ig domain unfolding at each interval is given by $p=j \cdot \alpha(F) \cdot \Delta t$, where $j$ is the number of folded domains, $\alpha(F)$ follows Bell's formula, and $\Delta t$ is the time step. After an Ig domain unfolds, a contour length increase of $28 \mathrm{~nm}$ occurs, the number of folded domains decreases by 1 , and the process continues. The parameter $\Delta x$ is inversely proportional to unfolding force and has a major effect on the standard deviation of the UF distribution, while $\alpha_{0}$ does not affect the standard deviation (Table 2). For this reason, matching the deviation of the simulated UF distribution with AFM data gives a good estimate of $\Delta x$. The $\left(\alpha_{0}, \Delta x\right)$ pair that best reproduces the experimental UF distribution is taken as best estimates for the Ig domain of interest. 


\begin{tabular}{|c|c|c|}
\hline$\alpha_{0}$ & $\Delta \mathrm{x}$ & $\begin{array}{c}\text { STDEV of } \\
\text { unfolding forces }\end{array}$ \\
\hline $.005 \mathrm{~s}^{-1}$ & $0.25 \mathrm{~nm}$ & 27.0 \\
\hline $.005 \mathrm{~s}^{-1}$ & $0.35 \mathrm{~nm}$ & 17.2 \\
\hline $.005 \mathrm{~s}^{-1}$ & $0.45 \mathrm{~nm}$ & 12.3 \\
\hline
\end{tabular}

\begin{tabular}{|c|c|c|}
\hline$\alpha_{0}$ & $\Delta \mathrm{x}$ & $\begin{array}{c}\text { STDEV of } \\
\text { unfolding forces }\end{array}$ \\
\hline $.000005 \mathrm{~s}^{-1}$ & $0.35 \mathrm{~nm}$ & 17.3 \\
\hline $.00005 \mathrm{~s}^{-1}$ & $0.35 \mathrm{~nm}$ & 17.4 \\
\hline $.0005 \mathrm{~s}^{-1}$ & $0.35 \mathrm{~nm}$ & 17.1 \\
\hline $.005 \mathrm{~s}^{-1}$ & $0.35 \mathrm{~nm}$ & 17.2 \\
\hline
\end{tabular}

Table 2. Top: The distance from the folded state to the transition barrier ( $\Delta x)$ significantly impacts the standard deviation of unfolding forces. Bottom: The unfolding rate of a protein under no net external force $\left(\alpha_{0}\right)$ does not affect the standard deviation of unfolding forces. Therefore, $\Delta x$ can be estimated solely from the standard deviation of unfolding force distributions generated from Monte Carlo simulations. Both parameters affect unfolding force.

The question still remains: is Bell's formula a reasonable approximation for the effect of force on unfolding rate? Rearranging Bell's formula gives $F=\frac{k_{B} T}{\Delta x} \ln \frac{\alpha(F)}{\alpha_{0}}$, which shows that a linear relationship should exist between a given external force $F$ and the natural logarithm of $\alpha(F) / \alpha_{0}$. The validity of this relationship can be nicely tested with my AFM force clamp dataset. For force clamp studies, an Ig10 pentamer was tethered and stretched until a set force point is reached. At that point the cantilever stops its retraction from the slide surface to maintain a constant force level. When an Ig domain unfolds, the force in the system drops as the molecule's slack length is increased. The cantilever then retracts from the slide surface until the set force level is reached again. This process continues until all domains have unfolded. This technique allows for direct measurement of force-dependent unfolding rate. Table 3 presents the force clamp data for T16I Ig10 and Figure 4 shows that there is a good linear relationship between clamp force and $\ln \alpha(F) / \alpha_{0}$. 
T16I Ig10 Force Clamp Data

\begin{tabular}{|c|c|c|}
\hline Force Clamp & $\alpha(\mathrm{F})$ & $\ln \alpha(\mathrm{F}) / \alpha_{0}$ \\
\hline $55 \mathrm{pN}$ & $4.87 \mathrm{~s}^{-1}$ & 5.50 \\
\hline $60 \mathrm{pN}$ & $6.56 \mathrm{~s}^{-1}$ & 5.79 \\
\hline $65 \mathrm{pN}$ & $6.87 \mathrm{~s}^{-1}$ & 5.84 \\
\hline $70 \mathrm{pN}$ & $16.29 \mathrm{~s}^{-1}$ & 6.70 \\
\hline $80 \mathrm{pN}$ & $24.24 \mathrm{~s}^{-1}$ & 7.10 \\
\hline $85 \mathrm{pN}$ & $28.47 \mathrm{~s}^{-1}$ & 7.26 \\
\hline
\end{tabular}

Table 3: Force-dependent unfolding rates $(\alpha(F))$ and $\ln \alpha(F) / \alpha_{0}$ at various force clamps for T16I Ig10.

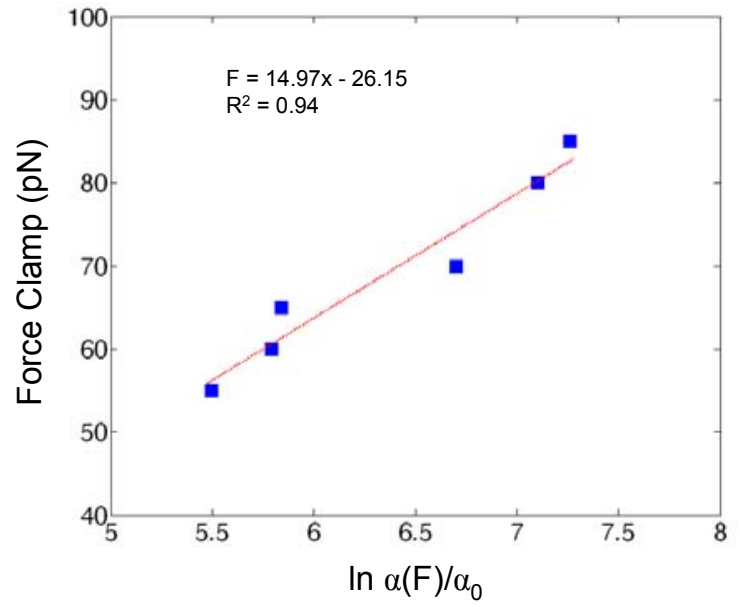

Figure 4: A linear relationship exists between the clamp force and $\ln \alpha(F) / \alpha_{0}$, which suggests that Bell's formula is a good approximation for the effect of force on unfolding rate.

The slope of this relationship equals $k_{B} T / \Delta x$, and from the linear fit we find that $\Delta x=0.27$ $\mathrm{nm}$, which is very close to the $\Delta x$ determined from comparing $\mathrm{MC}$ simulations with $\mathrm{AFM}$ data $(0.3 \mathrm{~nm})$. Admittedly, this method is not perfect: notice that the y-intercept from the linear fit does not match what is expected for $F=0$. However, this method does a surprisingly good job of describing force-dependent unfolding in Ig domains. This is likely due to the fact that Ig domains usually unfold in a single step from the globular structure to a random coil polypeptide. This all-or-nothing unfolding behavior is characteristic of proteins with short $\Delta x$ values. Large $\Delta x$ values indicate that the protein can withstand large displacements along the reaction coordinate of interest (such as the distance between two amino acids) prior to reaching the transition barrier that separates a protein's folded and unfolded conformations. 


\section{Beyond Single Molecules}

While single molecule spectroscopy is a powerful tool for studying the primary effects that mutation and phosphorylation have on protein mechanics, the behavior of an isolated protein is not necessarily indicative of how the protein behaves at the tissue level. In the case of titin, the three dimensional myofilament lattice is a protein-dense environment that is constantly changing as sarcomeres expand and contract. Although the enormous size of titin has precluded in-depth investigation of each of its subdomains, it is apparent that titin associates with numerous binding partners and signaling pathways. As the field of titin research continues to expand, one thing is clear-we have only just begun to understand the myriad functions that titin performs in the heart. This reservoir of untapped knowledge makes titin research both exciting and daunting.

The majority of my graduate research has investigated the link between a mutation in Ig10 (T16I) and the primary heart muscle disorder arrhythmogenic cardiomyopathy (AC). AC is characterized by the breakdown of healthy cardiac myocytes and subsequent replacement by fat and fibrous tissue. Various desmosomal mutations have been causally linked to AC [27], but the T16I mutation in Ig10 is the first sarcomeric protein mutation to be implicated in AC. The mutation (C39453T in the titin gene) showed complete segregation with the AC phenotype in affected individuals and provided strong genetic evidence that the mutation is linked to the disease.

I showed at the single molecule level that the T16I mutation weakens the mechanical stability of Ig10 and increases Ig10 degradation in vitro (Appendix A). In order to gain insights into the potential disease mechanism that links the mutation to the 
AC phenotype, my lab generated a genetically-engineered mouse model with the T16I mutation knocked-in (KI) to the titin gene (Figure 5).

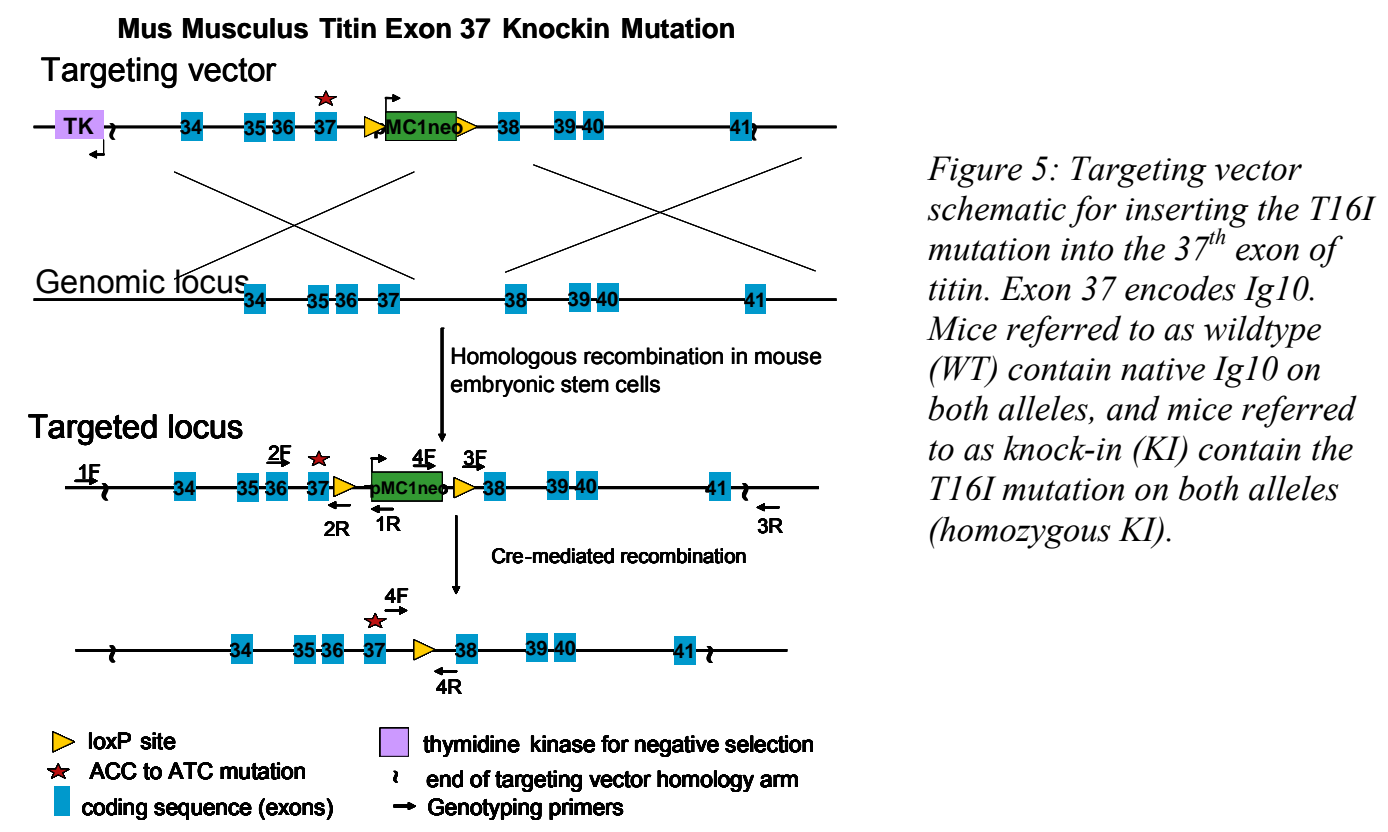

Based on my single molecule results, I hypothesized that the disease-linked titin mutation leads to the $\mathrm{AC}$ phenotype (fibrofatty replacement of myocardium) via a titin degradation mechanism. My AFM data showed that the presence of T16I significantly reduces the force needed to unfold Ig10 and increases its unfolding rate by a factor of four. I also performed a variety of in vitro degradation assays with recombinant Ig7-13 fragments and Ig10 pentamers that showed that the presence of T16I in Ig10 increases the rate at which Ig10 is cleaved by proteases (Appendix A), presumably due to disrupted local structure (i.e. loss of native protein contacts) at the mutation location. This increased degradation could also be due to the mutation increasing the amount of time that $\operatorname{Ig} 10$ occupies a protease accessible conformation without altering the native structure drastically. At the level of the sarcomere, any break in the titin chain would completely abolish titin's ability to generate passive forces. The passive force generated by stretched 
titin molecules are crucial for keeping thick filaments centered in the sarcomere [1], and degradation of titin leads to M-band misalignment [2]. Titin has also been implicated in cell signaling pathways (see section 7 in Appendix B), and it is likely that a broken titin molecule would negatively impact the signaling pathways associated with titin. Because titin is so large, accelerated titin turnover due to the T16I mutation may place unprecedented burden on the protein synthesis machinery of the cell and could disrupt the translation of other cellular proteins. For example, a rough calculation based on the transcription rate of RNA polymerase ( $\sim 3.9$ kilobases $/ \mathrm{min})[28]$ and translation rate of ribosomes (18 amino acids/sec)[29] shows that the creation of a single titin molecule probably takes at least 100 minutes (this does not include RNA splicing, which probably takes on the order of minutes). A lot of cellular resources and energy are needed for titin synthesis, which does not even include the effort needed to transport and integrate titin molecules into the protein-dense sarcomere environment. Not much is known about titin turnover in the cell, but it clearly is a complex and demanding process and may contribute to the mechanism that links the AC mutation to the disease phenotype. The generation of a mouse model with the T16I mutation knocked-in (KI) allows me to study the potential mechanism that links the titin mutation to the AC phenotype, but first it must be shown that the KI mice develop AC.

Searching for the AC Phenotype in the Mouse Model

A causal linkage between a mutation and disease in humans does not guarantee that the same mutation in a different organism will also lead to disease. Mice are most often used when genetically engineering a mammalian disease model because of their 
short gestation period ( 3 weeks) and relatively low cost. However, there are obvious differences between humans and mice, including that the mouse heart beats ten times faster than human hearts, mouse hearts contain less fat than human heats, and mice are extremely resilient creatures.

Under baseline conditions the KI mice do not show a disease phenotype. They reproduce normally and appear healthy and active. This is not surprising, since in humans the AC phenotype is characterized by a "concealed phase" [30] in which affected individuals are asymptomatic and present with little or no cardiac pathology. The disease progresses with age and often time is not diagnosed until post-mortem. In the family harboring the Ig10 T16I mutation, three of the seven affected family members required heart transplants at age 57 or older, two died from heart failure in their 60s, and two are stable ( 36 and 72 yrs). For these patients, the average age of disease onset was 36 years. Endurance athletes have been implicated as having a high risk to develop AC, and it is speculated that this is because endurance sports are associated with long-lasting volume overload of the right ventricle [31]. For these reasons, I decided to subject 10 month old male mice to a five week treadmill exercise regimen to try to instigate expression of the disease phenotype. After the treadmill regimen was completed (WT and KI mice did not show differences in running capacity) the geometry and pump function of the mouse hearts were determined by echocardiography (echo; Dr. Kirk Hutchinson performed the echo experiments). The four groups studied by echo were WT Exercised, WT Sedentary, KI Exercised, and KI Sedentary. Mitral valve A wave velocity (which measures the blood flow as the left atrium contracts to squeeze out any remaining blood into the left ventricle) was significantly reduced in both exercise groups compared to sedentary WT 
mice and was trending towards significance in sedentary KI mice compared to sedentary WT (Figure 6). A lower mitral valve A wave velocity indicates that the atrial kick is not as strong, which may be due to a stiffer left ventricle (LV). In addition, the mitral valve deceleration time was significantly shorter in sedentary KI mice compared to sedentary WT mice (Figure 6). Reduced mitral valve deceleration time implies that the LV is stiffer, and these two echo parameters being reduced could indicate restrictive filling in the KI mice.
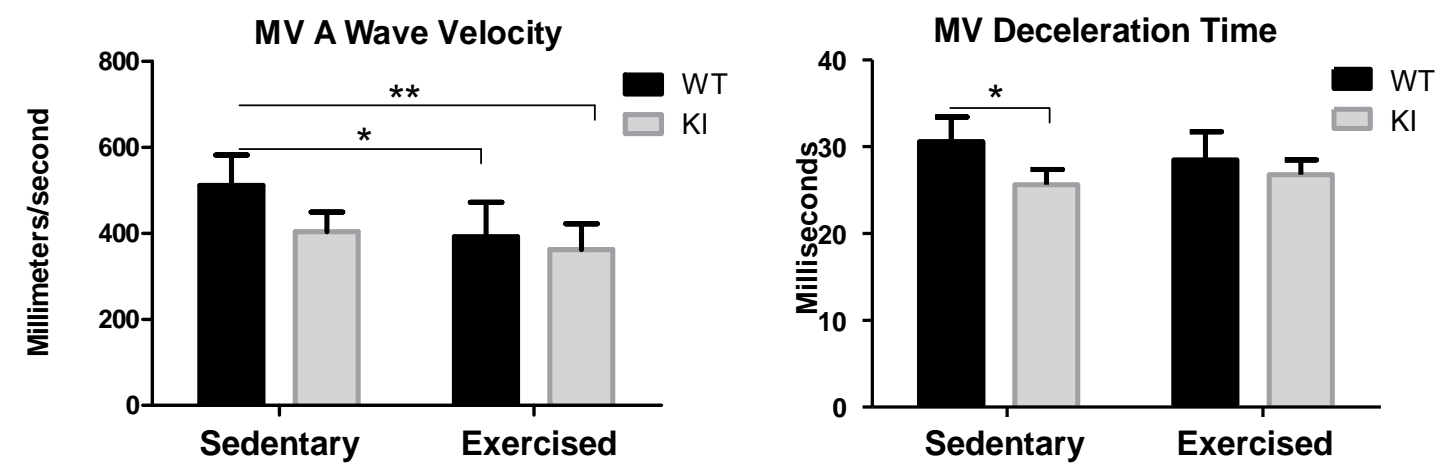

Figure 6: Left-The velocity of the mitral valve A wave is reduced in WT and KI mice after five weeks of treadmill running compared to sedentary WT mice. KI sedentary mice also have a lower A wave velocity, but this difference is not yet significant. Right-Sedentary KI mice have a shorter mitral valve deceleration time compared to sedentary WT mice, which implies a stiffer left ventricle. Significance was determined using two-way ANOVA and Tukey's post-hoc test. $N=6$ for all groups. ** $p<0.01, * * * p<0.001$.

After echo, the mice were then sacrificed and their hearts were excised. Cross sectional cuts ( $\sim 2 \mathrm{~mm}$ thick) were made through the right ventricle (RV) and left ventricle (LV) of the heart for histology studies. One cross section slice was fixed in 3\% glutaraldehyde for picrosirius red (PSR) staining, and one slice was snap frozen in OCT for oil red O staining. PSR stains linearized collagen fibers and presents as bright orange/yellow birefringence under polarized light. Increased collagen volume fraction in the heart indicates fibrosis. Oil red O stains adipose tissue. Since a hallmark of arrhythmogenic cardiomyopathy is myocardial infiltration by fibrous and fat tissue, I wanted to determine 
if this phenotype was present in the KI mice and if long-term volume overload due to treadmill running exacerbated the phenotype. Figure 7 shows bright collagen fibers after PSR staining. Two-way ANOVA shows no significant difference between the four groups, although the variance due to the interaction factor is nearly significant $(\mathrm{p}=0.06)$. This interaction factor is lessened $(\mathrm{p}=0.10)$ when the outlier in the exercised WT dataset is removed.
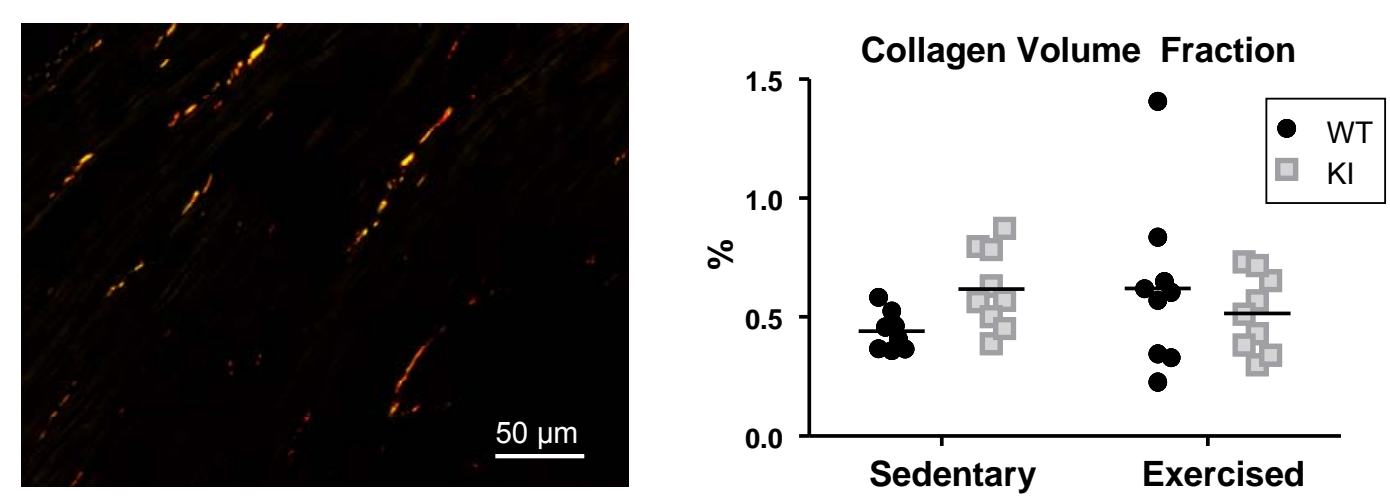

Figure 7: Left-Collagen fibers appear as bright strands upon PSR staining. Right-The collagen volume fraction is determined from the percentage of collagen present in cross sectional slices of the heart. No significance between groups was seen using two-way ANOVA.

Figure 8 shows fat deposits stained with oil red $\mathrm{O}$. There was no significant difference between groups, although exercised WT mice exhibited less fat content while exercised KI mice had more fat relative to the respective sedentary group. The fact that exercise influences the amount of cardiac fat in opposite ways can be seen by the variance due to the interaction factor being significant $(p=0.03)$. Although speculative, it is interesting to suggest that the lower fat percentage in the exercised WT mice compared to sedentary WT mice is the expected, beneficiary physiological response to exercise while the increase in fat in the exercised KI mice compared to sedentary KI mice is indicative of the AC phenotype beginning to show itself due to exercise-induced volume overload. 

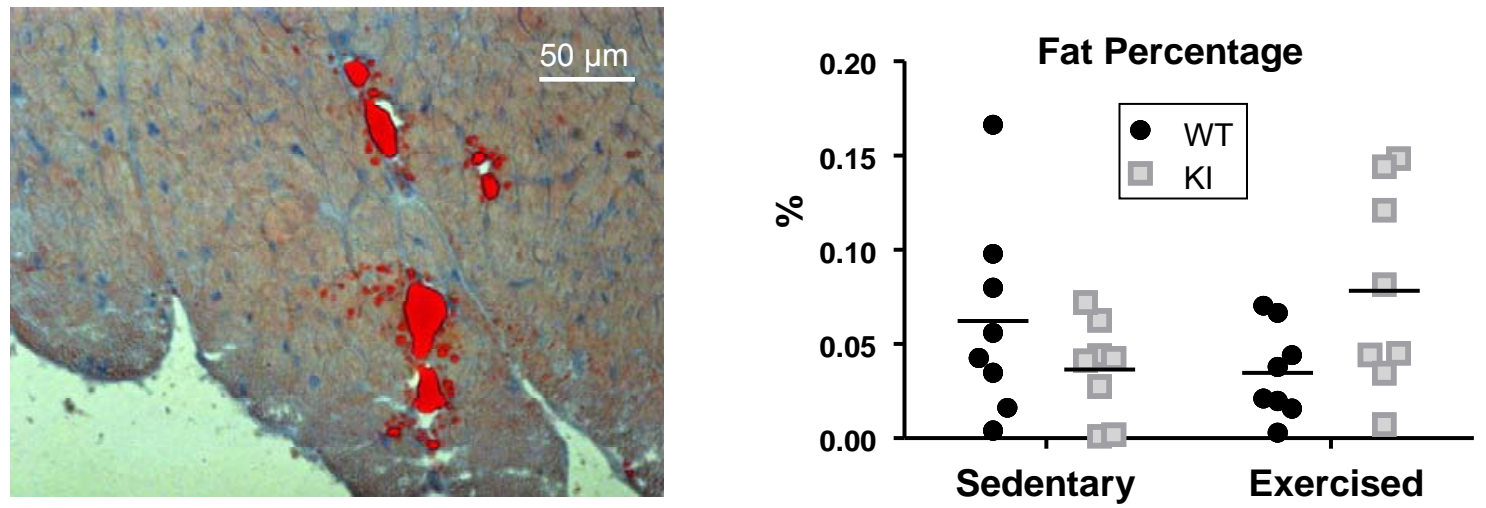

Figure 8: Left-Fat appears bright red after staining with oil red $O$. The percentage of fat was determined by the total area of fat divided by the total cross section of the heart slice. Right-There is no significant difference amongst the four groups studied, although the variance due to the interaction factor is significant $(p=0.03)$.

The same fixed heart slices used for PSR staining were also used in an assay to detect apoptotic cells (cells undergoing programmed cell death). It is thought that the fibrofatty pathology seen in $\mathrm{AC}$ patients occurs after myocardial degradation, and apoptosis is one pathway that would lead to a reduction of myocytes in the heart. This assay detects the presence of cleaved caspase 3, a central protease in the apoptosis pathway that is often used as an apoptotic marker. Unfortunately, apoptotic cells were not seen in my mouse heart samples.

Since my hypothesis is that the link between the T16I mutation and the AC phenotype involves titin degradation, I looked at titin isoform expression and degradation with $1 \%$ agarose gel systems specialized for resolving giant proteins. These gels allow for quantification of full-length cardiac titin isoforms (N2BA and N2B) as well as titin degradation products. Extensive gel analysis with the help of Mr. Chandra Saripalli was performed on a large set of 4 month old female WT and KI mice (Figure 9). The KI mice have less degraded titin in both the left and right ventricle compared to WT mice. This result was surprising since I expected the presence of the T16I mutation to increase titin 
degradation rates (such as what is seen at the single molecule level). However, protein turnover mechanisms in the cell are quite complex and this reduced level of degraded titin in the KI tissue could be due to a variety of factors. This needs to be studied further in the future.
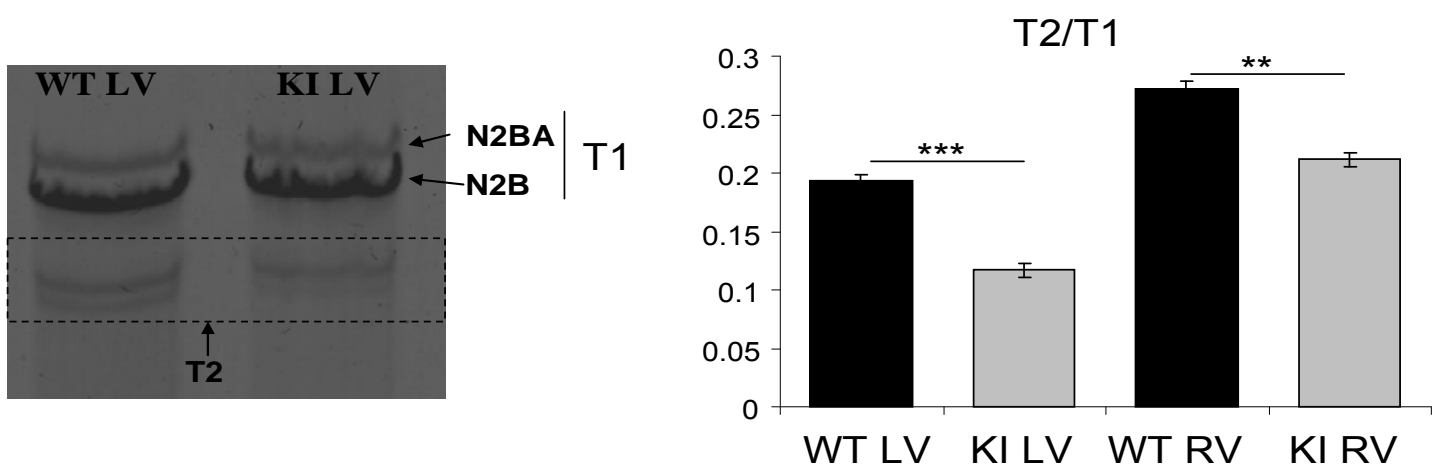

Figure 9: Left-Titin gels allow for quantification of full-length titin isoforms (T1) and titin degradation products (T2). Right-T2/T1 ratio is significantly reduced in the LV and RV of KI mice. This either indicates that $T 1$ is degraded less rapidly in KI mice or that T2 is degraded more rapidly.

\section{Future Directions}

There is an acute need for improved methods to analyze unfolding force distributions to estimate parameters such as unfolding rate, the distance along the reaction coordinate from the folded state to the transition barrier, and the height of the activation barrier. Theories have been developed to improve such analyses that take into account force-dependent loading rates (which is the case in constant speed pulling experiments when flexible linkers are present) and explicit free energy surfaces (force dependent unfolding rates and probability distributions can be evaluated analytically for simple energy profiles such as a linear-cubic surface) [32]. An untested assumption of these theories is that the process of irreversible unfolding as a function of external force is quasi-adiabatic, that the characteristic relaxation time of the system in the folded state is much faster than the escape over the activation barrier separating folded and unfolded 
conformations [33]. If the adiabatic assumption is correct, the theory predicts that forcedepending unfolding rates should be independent of pulling speed:

$$
\alpha(F)=\frac{k_{s} v p(F \mid v)}{1-\int_{0}^{F} p\left(F^{\prime} \mid v\right) d F^{\prime}} \text { for all } v, \text { where } k_{s} \cdot v \text { is the loading rate }(\mathrm{pN} / \mathrm{s}) \text { and } p(F \mid v) \text { is the }
$$

probability of unfolding at a force $F$ given the pulling speed $v$ [32]. This implies that $\alpha(\mathrm{F})$ determined from different pulling speeds should collapse onto a master curve. This behavior is seen with my simulated UF data for pulling speeds covering four orders of magnitude (Figure 10).

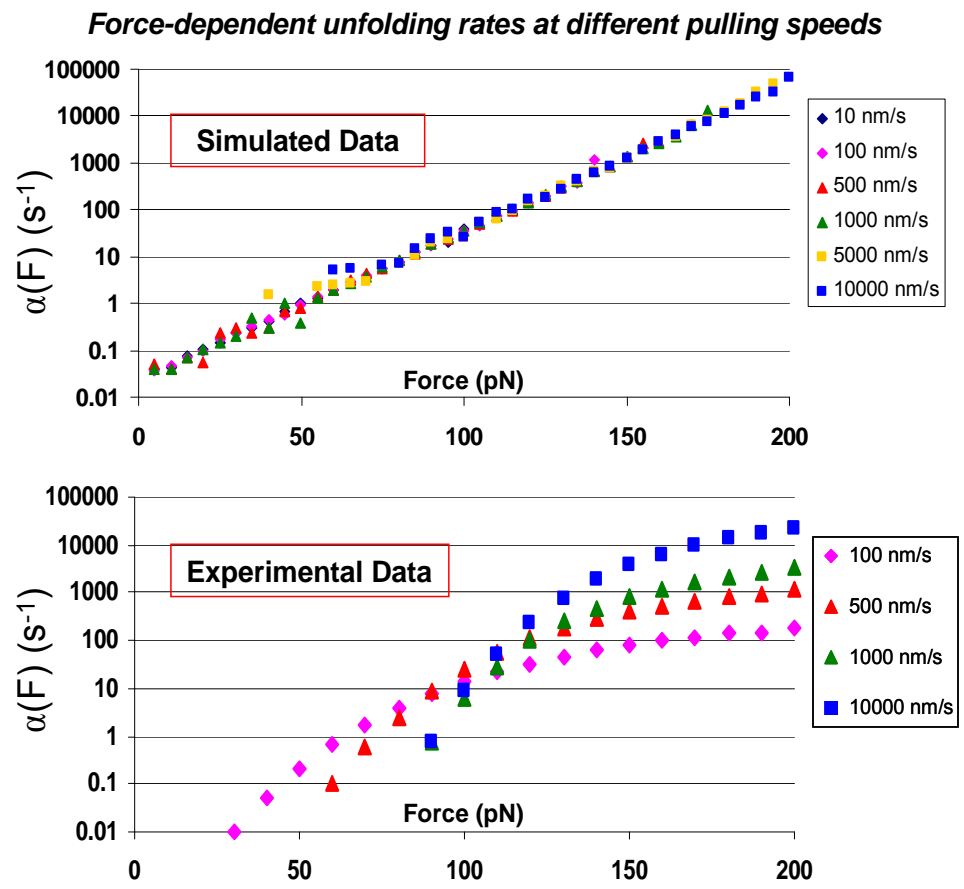

Figure 10: For protein unfolding under monotonically increasing external force, the survival probability $(S(t)$; the probability that the protein is still folded at time t) is related to the unfolding rate by $\dot{S}(t)=-k(F(t)) S(t)$. Under the quasi-adiabatic assumption the unfolding rate is independent of pulling speed. Using the $p(F \mid v)$ from my simulated and experimental UF data and a force-dependent loading rate (due to non-linearity of wormlike chains) I plotted $\alpha(F)$ for all forces probed by various pulling speeds.

The fact that my AFM data from different pulling speeds does not overlap most likely implies that Ig10 unfolding involves intermediate unfolding barriers and multiple unfolding pathways [34]. My AFM data suggests that two-step unfolding sometimes occurs (partial refolding events were seen during refolding experiments and two-step 
unfolding was occasionally seen during force clamp experiments), but these events were too infrequent to generate a robust two-step unfolding dataset.

Improved accuracy of $\alpha(\mathrm{F})$ estimation can be achieved by performing force clamp experiments with lower force clamps. This would allow testing of the applicability of Bell's formula over a larger force range and, more importantly, could directly determine $\alpha(\mathrm{F})$ at low (perhaps approaching physiological) forces. These experiments would require very low AFM drift and stable binding between the protein fragment and cantilever tip since unfolding rates are much slower at low force.

A lot of work is still needed to determine how the T16I mutation leads to AC (and if our mouse model even exhibits the classical disease phenotype). At this point my hypothesis remains that the link between the mutation and the $\mathrm{AC}$ phenotype is related to titin degradation. The decreased T2/T1 ratio in KI mice (Figure 9) suggests that titin turnover dynamics are different in the KI mice, but this needs to be investigated further. I have done preliminary assays to determine proteasome activity in the WT and KI tissue and found that proteasome activity is $15 \%$ less in the $\mathrm{KI}$ animals $(\mathrm{n}=2)$. This initial result was surprising because increased protein turnover would correspond to increased proteasome activity, but more work is needed to elucidate the rates of titin synthesis and titin degradation in cardiac tissue.

Perhaps the most direct way to probe the effect that the T16I mutation has on titin degradation in intact sarcomeres is with myofibril mechanics. Myofibrils are long, cylindrical structures in muscle that are composed entirely of serially-linked sarcomeres. Single myofibrils can be isolated by blending muscle tissue and can subsequently be tethered between a micromanipulator-controlled glass needle and cantilever. The glass 
needle is attached to a piezotube that allows for quick length changes of the myofibril. Force in the system is measured by a quadrant photodetector that receives laser light that deflects off the cantilever, very similar to the optical detection system used in AFM, except that now horizontal forces can be measured instead of vertical forces. With this experimental setup, I can stretch myofibrils and then flow through protease-rich solution that will degrade titin. This allows for direct measurement of force-dependent degradation (titin degradation will be determined by a reduction in titin-based passive stiffness in the stretched myofibril). My hypothesis is that titin molecules in myofibrils from the KI mice (with the T16I mutation present in every titin molecule) will be degraded more rapidly compared to WT myofibrils, which will be measured by a faster reduction in titin-based passive force upon the introduction of proteases. This novel experiment will be important for showing that the T16I mutation has a primary effect on Ig10 structure and stability in full-length, sarcomere-integrated titin molecules.

It is possible that the disease phenotype has not been apparent because the animals we have studied are too young (treadmill mice were $\sim 10$ months old at time of dissection). It is well-known that the $\mathrm{AC}$ phenotype is age-dependent, and future experiments will be performed on 18 month old WT and KI male mice. These mice will be echoed, and upon dissection the heart tissue will be used for histological studies, titin isoform gels, and proteasome assays. Depending on the echo results, additional studies may also be performed.

Currently, our collaborators at UCSD (the lab of Dr. Farah Sheikh) are performing extensive Western Blots on LV and RV tissue from the mice used in the treadmill exercise study. They are looking at the protein levels of desmosomal proteins 
because $\mathrm{AC}$ has been causally linked to various desmosomal mutations [31].

Desmosomes, along with gap junctions and fascia adherens junctions, make up the intercalated disks that hold cardiomyocytes together and allow for mechanical coupling and intercellular signaling. Interestingly, a recently-defined subcellular structure called the transitional junction connects myofilaments (including titin) and the intercalated disk [35], which may offer insights regarding how the T16I mutation in titin leads to a disease phenotype that mimics those often associated with desmosomal mutations.

The link between the T16I mutation and AC is not clear, but the generation of the T16I KI mouse model is a valuable tool for studying the disease mechanism. This mouse model would not have been made if not for the strong single-molecule studies that showed a clear effect of the T16I mutation on Ig10 mechanics and stability. I think performing single molecule studies in conjunction with cell, tissue, or organism level studies is crucial for achieving a robust understanding of biophysical processes. 
7. Investigating the link between the T16I Ig10 mutation and arrhythmogenic cardiomyopathy using a knock-in (KI) mouse model (Manuscript in Progress)

Introduction

The giant protein titin is the third myofilament of the sarcomere, helps determine diastolic stiffness, and is crucial for sarcomere organization. Mutations in titin have been associated with dilated cardiomyopathy [36], but recently a mutation in titin was linked with arrhythmogenic cardiomyopathy (AC) for the first time [37]. AC has been termed a "disease of the desmosome" because numerous desmosomal mutations have been identified that cause the disease phenotype [31]. The primary phenotype is identified by replacement of the myocardium with fibrofatty tissue, which leads to problems with electrical conduction and arrhythmias early on and reduced contractility and heart failure as patients age.

Genetic analysis of 38 well-characterized AC families identified a C39453T nucleotide transition in the $37^{\text {th }}$ exon of titin that showed complete segregation with the AC phenotype in six affected patients [37]. In addition, two fifth-degree relatives with AC shared this mutation, which strongly suggests that the mutation is linked with the disease phenotype. The $37^{\text {th }}$ of titin's 363 exons codes for the $10^{\text {th }}$ immunoglobulin(Ig)like domain of titin's elastic I-band region (Ig10; Figure A1). The AC-linked mutation changes the native threonine residue into isoleucine (T16I), and single molecule studies have shown that the mutation significantly weakens the mechanical stability of Ig10 and increases its rate of unfolding [20]. This study also showed that the T16I mutation increases Ig10 degradation in vitro due to changes in the structure of $\operatorname{Ig} 10$ local to the 
mutation location. This single molecule evidence suggested that the cellular mechanism that links the titin Ig10 mutation to AC involves accelerated titin degradation rates, which is consistent with the fact that a primary AC phenotype is breakdown/replacement of ventricular myocardium.

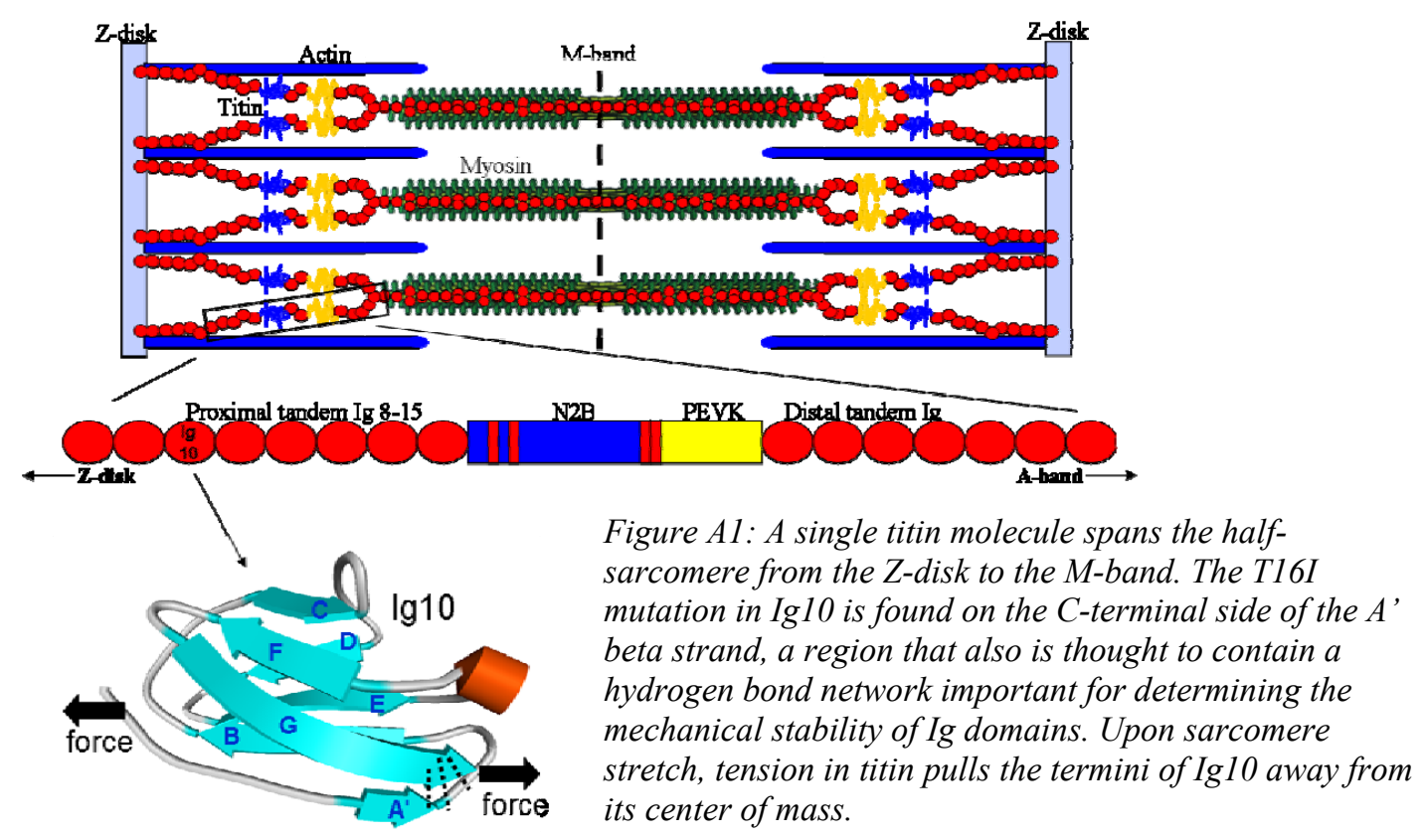

To investigate the molecular mechanisms that lead to the disease, a genetically engineered mouse model was created with the Ig10 mutation knocked-in. Mice with the T16I mutation present on both alleles (homozygous knock-in animals) were used in this study and are termed T16I KI mice. Although the T16I mutation has a clear effect on recombinant Ig10 polyproteins at the single molecule level [20], these studies cannot replicate the conditions that titin experiences in vivo. With a mouse model in hand, we can study the effects that T16I has on Ig10 in full-length titin molecules that are properly integrated into the protein dense sarcomere environment. The goal of this study is twofold: 1) to determine if the T16I mutation causes the arrhythmogenic cardiomyopathy 
phenotype in homozygous KI mice and 2) to gain insights into the potential molecular mechanisms responsible for development of the disease.

Experimental Procedures

Generation of Mouse Model —A gene targeting strategy was employed to introduce an ACC to ATC point mutation in exon 37 of the mouse titin gene in order to mimic the T16I Ig10 human mutation. The targeting vector was built using a vector backbone carrying neomycin resistance for a positive selection. The targeting vector consists of two arms of homology. The short arm of homology was $3.3 \mathrm{~kb}$ in size and carries the point mutation, and the long arm of homology was $4.1 \mathrm{~kb}$. The point mutation was introduced using a site-directed mutagenesis kit (Agilent, Santa Clara, CA) and its presence was confirmed by sequencing. The targeting vector DNA was electroporated into 129S6 embryonic stem cells. The neomycin resistant clones were screened by PCR using primers outside the homologous arms and neomycin primers in order to select the clones that have taken the targeting DNA by homologous recombination. Mice carrying the mutation were genotyped by PCR across the mutation, using a forward primer on the 5' end of the mutation and a neo reverse primer. The PCR amplified bands were cut out from the gel, purified, and sent for sequencing to prove the presence of the mutation.

Mouse genotyping-Mouse tails were digested in tail lysis buffer and incubated at $55^{\circ} \mathrm{C}$ overnight. Primers were added and a standard PCR cycling protocol was performed. PCR products were determined by capillary electrophoresis using an Applied Biosystems 3730 DNA Analyzer, with the WT amplicon at 253 bp and the T16I KI amplicon at $330 \mathrm{bp}$. 
Exercise Protocol_-Age-matched male mice were randomly selected for treadmill exercise similar to published protocols [38]. The mice were acclimated to the treadmill environment (Exer 3/6, Columbus Instruments, Columbus $\mathrm{OH}$ ) for one week (at low to moderate running speeds) before the five week exercise protocol began. The exercise protocol was 60 minutes and always began at a low speed $(5 \mathrm{~m} / \mathrm{min})$ with stepwise speed increases every 3-6 minutes until a maximum velocity was reached at $27.5 \mathrm{~m} / \mathrm{min}$. Mice were removed from the treadmill upon exhaustion (when the mice rested on the shock grid) or until the entire protocol was complete. Treadmill exercise took place in the morning each day.

Echocardiography- Mice were echoed under 1.0\% isoflurane anesthesia (USP, Phoenix) in 100\% oxygen. Transthoracic echo images were obtained with a Vevo 2100 High Resolution Imaging System (Visual-Sonics, Toronto) using the model MS250 scan head designed for murine cardiac imaging. Care was taken to avoid animal contact and excessive pressure. Images were collected and stored as a digital cine loops for offline analysis (Vevo 2100 software suite). Standard imaging planes, m-mode, Doppler, and functional calculations were obtained according to American Society of Echocardiography guidelines [39]. The short-axis view was used for mmode acquisition of percent fractional shortening and ventricular geometry. Left atrium dimensions were measured in the parasternal long-axis view directly below the aortic valve leaflets. Passive LV filling peak velocity, atrial contraction flow peak velocity, and E and A wave velocities were determined from the images of mitral valve Doppler flow from apical four-chamber view. Mouse heart rates were maintained between 500-550 bpm for M-mode and 350-450 bpm for Doppler studies. 
Histology-After cutting off the apex of the heart, $2 \mathrm{~mm}$ thick cross sectional slices were made through the left and right ventricles. The slice closer to the apex of the heart was snap frozen in OCT using an isopentane boat on liquid nitrogen. From this slice, three step sections (separated by $\sim 100$ um) were cut and stained with oil red $\mathrm{O}$ for detection of fat droplets. The second $2 \mathrm{~mm}$ thick slice was fixed in 3\% glutaraldehyde. Step sections were cut from this slice and stained with picrosirius red, which stains linearized collagen fibers. The percentage of fat accumulation in the heart slices was determined by the fractional area of oil red $\mathrm{O}$ stained tissue normalized by the total cross sectional area of the heart slices using a Zeiss AxioImager M1 microscope and AxioVision 4.7.2 software. Similarly, the collagen volume fraction of the heart slices was determined by the percentage of the heart slice occupied by picrosirius red-stained collagen fibers that appear as bright birefringence under polarized light.

For identifying apoptotic cells in the mouse hearts, cross sectional slices through the ventricles were fixed in $10 \%$ neutral buffered formalin for 24 hours, processed, and embedded in paraffin. Routine hematoxylin and eosin (H\&E) stains were performed on sections of tissue cut with a width of 3 microns from the formalin fixed, paraffinembedded blocks. Immunohistochemistry for Cleaved Caspase 3 (clone D175) was performed using rabbit monoclonal antibodies (\#9661S, Cell Signaling Technology, Danvers, MA), with human tonsil tissue used as the positive tissue controls. Mouse spleen tissue sections were stained with a Discovery XT Automated Immunostainer (Ventana Medical Systems, Tucson, AZ) using reagents for deparaffinization, cell conditioning (antigen retrieval with a borate-EDTA buffer), primary antibody staining, detection, and amplification using a biotinylated-streptavidin-HRP and diaminobenzidine 
system and hematoxylin counterstaining. Following staining on the instrument, slides were dehydrated through graded alcohols to xylene and coverslipped with mounting medium.

Skinned Tissue Mechanics — Papillary muscles from the LV were dissected and skinned overnight in skinning solution (in mM: BES 40, EGTA 10, $\mathrm{MgCl} 2$ 6.56, Na-ATP 5.88, DTT 1, K-propionate 46.35, creatine phosphate 15, E-64 0.1, leupeptin 0.4, and PMSF 0.5 , and $1 \%$ Triton X-100) . After skinning, the tissues were washed with Tritonfree relaxing solution and then transferred to a $50 \%(\mathrm{v} / \mathrm{v})$ solution of glycerol and relaxing solution. Tissues were stored at $-20 \mathrm{C}$. Small fibers $\left(\sim 0.05 \mathrm{~mm}^{2} \mathrm{CSA}\right.$ and $\sim 1 \mathrm{~mm}$ long) were dissected along the long axis of the papillary muscle and clipped with aluminum T-clips. Fibers were attached to a force transducer (AE801, SensorOne, Sausalito, CA) and motor (308B, Aurora Scientific, Aurora, Canada). Cross sectional area was calculated using the length and width of the muscle strip and assuming an ellipsoid shape. Mechanical protocols were performed in relaxing solution at room temperature. Muscle strips were stretched using a stretch-hold-frequency sweep protocol at a speed of $10 \%$ length $/ \mathrm{sec}$. After stretch to a given sarcomere length, the stretched tissue was held for 90 seconds to allow for passive tension to reach steady state, and then a frequency sweep of $0.1-100 \mathrm{~Hz}$ at $2 \%$ amplitude was performed. A 15 minute rest period was allowed between each stretch. To extract the relative contributions of the extracellular matrix and titin to total passive force, thick filaments were depolymerized with $\mathrm{KCl}(1 \mathrm{M})$ and thin filaments were depolymerized with $\mathrm{KI}(0.6 \mathrm{M})$. The stretchhold-frequency sweep protocol was then repeated, with the resulting passive forces 
attributed to the ECM and the difference between the total force and ECM force defined as titin-based passive tension.

Titin Quantification Gels-Cardiac tissues were quick frozen in liquid nitrogen and finely ground with glass pestles cooled in liquid nitrogen. Pulverized tissues were allowed to warm to $-20^{\circ} \mathrm{C}$ and then suspended in $50 \%$ urea buffer (in M: urea 8 , thiourea 2, Tris-HCl 0.05, DTT 0.075, with 3\% SDS and 0.03\% bromophenol blue) and 50\% glycerol with protease inhibitors (in mM: E-64 0.04, leupeptin 0.16, and PMSF 0.2). Tissues were solubilized for four min at $60^{\circ} \mathrm{C}$ and then incubate for 10 more minutes at $60^{\circ} \mathrm{C}$. After cooling, solubilized samples were centrifuged for $5 \mathrm{~min}$ at $13 \mathrm{k} \mathrm{rpm}$; the supernatant was loaded into 1\% vertical agarose gels. Each gel was run for $3 \mathrm{hr} 20 \mathrm{~min}$ at $15 \mathrm{~mA}$ and then Coomassie stained. Protein bands were quantified using One-Dscan (Scanalytics Inc, Rockville, MD). Titin isoform and myosin heavy chain optical densities were determined as a function of volume, and the linear relationship of protein versus loading volume was used to quantify protein expression ratios.

Western Blots-Proteins were separated by $0.8 \%$ agarose gels (titin) or SDSPAGE gels (recombinant protein), transferred to PVDF membranes and Ponceau S stained (Sigma) to determine protein levels. To determine the presence of WT and T16I Ig10 the membranes were blotted with custom made rabbit polyclonal antibodies raised against the Ig10 peptides (GL Biochem Ltd., Shanghai). Secondary antibodies conjugated with fluorescent dyes with infrared excitation spectra (Biotium, Hayward, CA) were used for detection with the Odyssey Infrared Imaging System (LI-COR Biosciences, Lincoln, NE) and analyzed densitometrically. 
Results and Discussion

Sequencing confirmed the presence of the T16I mutation at the DNA level (see Experimental Procedures). In addition, quantitative reverse transcriptase PCR was performed on left ventricular tissue from heterozygous T16I KI mice and showed that equal levels of WT and T16I KI RNA were present, which shows that the mutation does not affect transcription of the titin gene, as expected.

The confirm that the T16I mutation is present at the protein level, we used antibodies raised against the WT and T16I Ig10 sequences. Because the sequences differ by only one amino acid, cross-reactivity was expected. The specificity of the respective antibodies was tested by performing Western Blots on recombinant Ig7-13 fragments (with either WT Ig10 or T16I Ig10 present as the central Ig domain in the 7 domain heteropolyprotein). The WT Ig10 antibody detects the Ig7-13 fragment with WT Ig10 15 times more strongly than the fragment with T16I Ig10 present. Similarly, the T16I Ig10 antibody detects the Ig7-13 fragment with T16I Ig10 present 7 times more strongly (Figure A2). The antibodies were less specific when used on full-length titin isoforms, most likely because there are many Ig domains in titin. For example, the I-band region of the N2B titin isoform has $\sim 40$ distinct (i.e. different primary sequences) Ig domains, while the N2BA titin isoform has additional Ig domains. Also, there are more than $50 \mathrm{Ig}$ domains in the Z-disk, A-band, and M-band regions in both the N2B and N2BA isoforms [40]. Nonetheless, the T16I Ig10 antibody detects titin molecules from the T16I homozygous KI mice $1.5 x$ more strongly than titin from WT mice, and the WT Ig10 antibody detects native titin molecules $2.4 \mathrm{x}$ more strongly than titin with the T16I mutation present (Figure A2). 

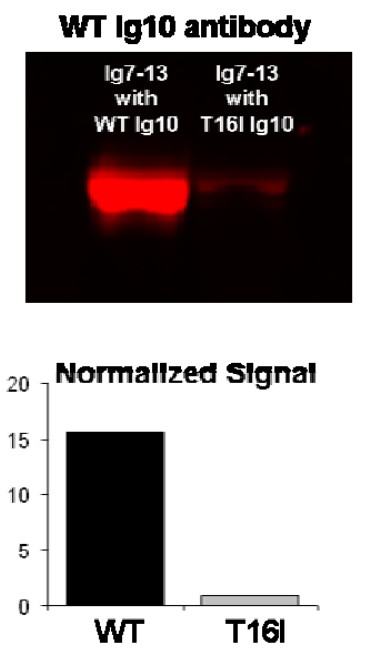
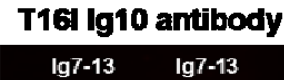

with with

$\begin{array}{ll}\text { WT Ig10 } & \text { with } \\ \text { T16l Ig10 }\end{array}$
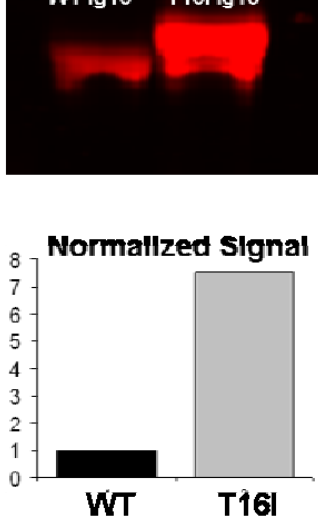

WT Ig10 antibody

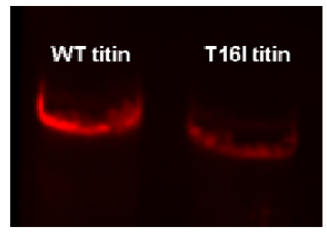

T161 lg10 antibody
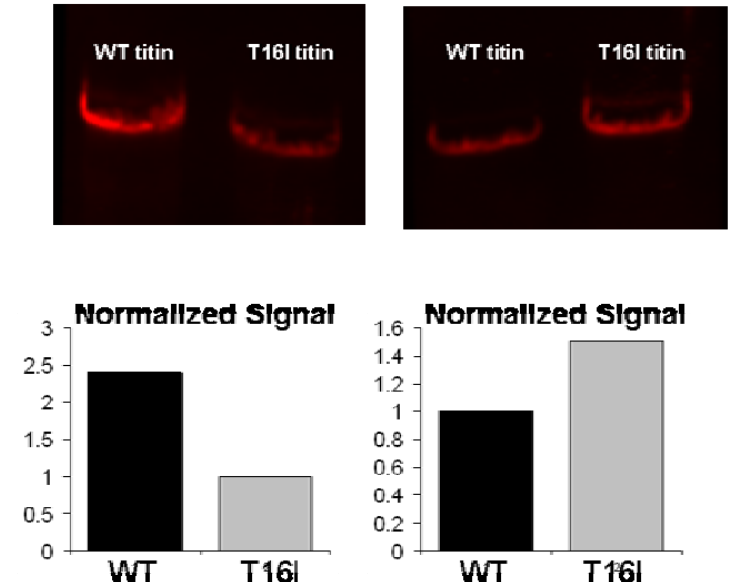

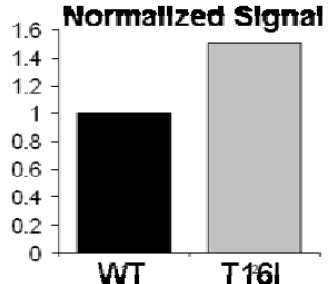

Figure A2: Western blots with WT and T16I Ig10 antibodies. The antibodies show more specificity when probing recombinant Ig fragments compared to full-length titin molecules, which was expected. The bar graphs represent the relative Western blot signals normalized by total protein levels as determined by Ponceau S staining.

The T16I KI mice breed and develop normally under baseline conditions. There are no morphological differences between WT and T16I KI female mice except for a shorter tibia length in the WT animals $(17.90 \mathrm{~mm}$ compared to $18.26 \mathrm{~mm} ; \mathrm{p}<0.001)$. To see if the mutation alters titin isoform expression or titin degradation in vivo we ran $1 \%$ agarose gels specialized for separating and visualizing large proteins [41] on all four heart chambers from four month old WT and T16I KI female mice (Figure A3). The $\mathrm{N} 2 \mathrm{BA} / \mathrm{N} 2 \mathrm{~B}$ ratio was not different between the WT and T16I mice in any chamber of the heart. This was expected because the presence of a single point mutation in a titin exon should not affect titin splicing, although its possible that N2BA and N2B degradation rates are differentially influenced by T16I since strain is higher in the shorter N2B titin isoform at a given sarcomere length. The T16I KI tissue did show a decrease in degraded titin compared to WT tissue, as shown by a reduced ratio of degraded titin to full-length titin. The reduction was significant in all chambers except for the left atrium. This result was surprising because our hypothesis was that the T16I mutation would lead to 
increased titin degradation, which would presumably lead to an increase in the ratio of degraded titin to full-length titin in the T16I KI mice. However, a reduction in titin degradation products could also be due to accelerated degradation of already degraded titin, i.e. once full-length titin (N2BA or N2B) is degraded initially it is subsequently degraded further and therefore no longer identifiable as a discernible protein band on the gel. For this reason it is unclear if these gel results indicate an overall increase or decrease in protein degradation rates in the T16I KI mice compared to WT.

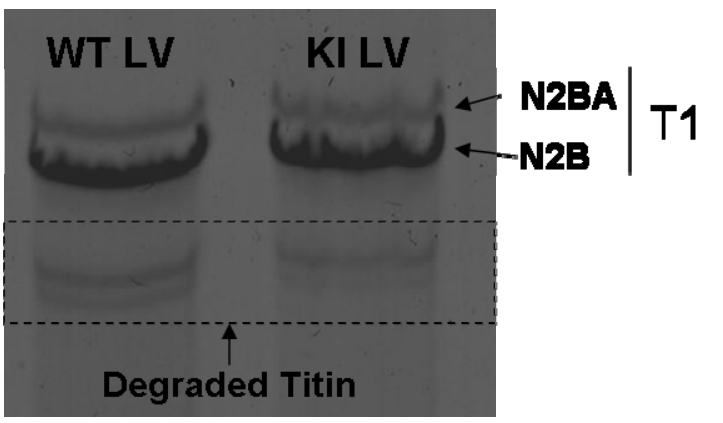

Figure A3: Left-Visualization of titin on 1\% agarose gels. Full-length (T1) and degraded titin can be quantified. Below: $N o$ differences in $N 2 B A / N 2 B$ ratio were seen in any chamber of the heart, but the T16I KI mice had significantly less degraded titin in the $L V, R V$, and $R A$. $N=10$ for all chambers. ${ }^{*} p<.05, * * p<.01$, $* * * p<.001$.
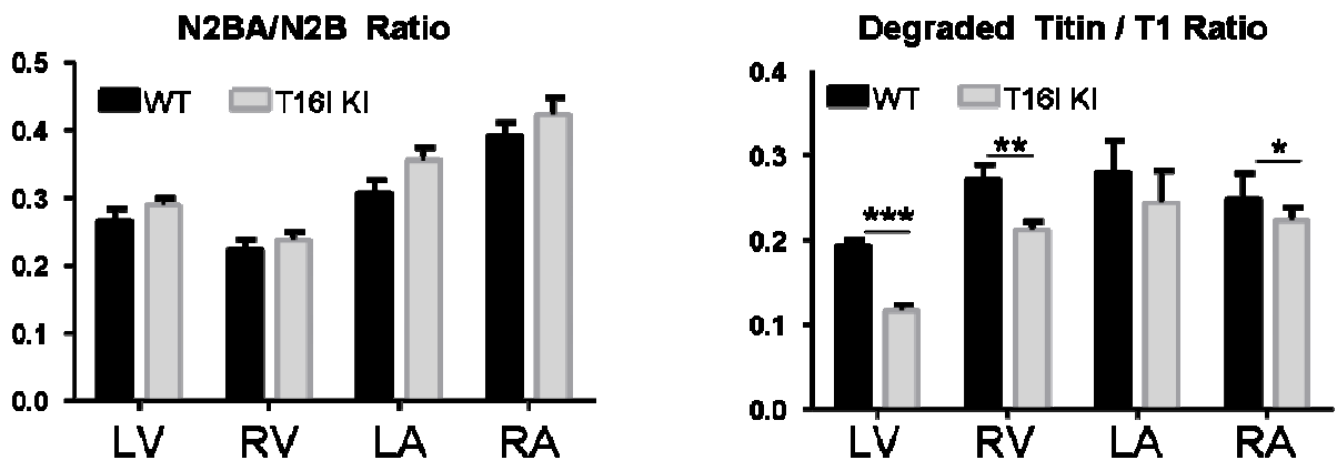

To determine if the T16I KI mice have a difference in degradation compared to WT mice, we will measure the proteasome activity in WT and T16I KI LV. The ubiquitinproteasome pathway is the major proteolytic system in eukaryotic cells and assaying for proteasome activity can give a general picture of the rate of protein turnover in cells. This work is in the preliminary stages. 
It was not unexpected that the T16I KI mice do not show a phenotype under baseline conditions because arrhythmogenic cardiomyopathy is often preceded by a concealed phase in which the disease phenotype is absent, especially in younger individuals [42]. To try to instigate development of the disease phenotype we subjected nine month old male mice to five weeks of treadmill exercise, as the increased pre-load associated with sustained exercise has been suggested to explain the reason that endurance athletes have a high risk to develop AC [31]. It has previously been shown that endurance training accelerates the disease phenotype in an AC mouse model deficient in the desmosomal protein plakoglobin [43]. After one week of acclimation, age-matched mice were treadmill exercised five days a week for five weeks. Physical exhaustion was defined by the mice remaining on the shock grid on the backside of the treadmill for five seconds consecutively, at which point the mice were removed. On average, $1 / 3$ of the mice were able to complete the entire treadmill regiment, and endurance did not correlate with genotype. Following the five week treadmill protocol the geometry and pump function of the exercised mice and a matching group of sedentary mice was studied using echocardiography (echo). Mitral valve A wave velocity (which measures the blood flow as the left atrium contracts to squeeze out any remaining blood into the left ventricle) was significantly reduced in both exercise groups compared to sedentary WT mice and was trending towards significance in sedentary T16I KI mice compared to sedentary WT (Figure A4). A lower mitral valve A wave velocity indicates that the atrial kick is not as strong, which may be due to a stiffer left ventricle (LV). In addition, the mitral valve deceleration time was significantly lower in sedentary T16I KI mice compared to sedentary WT mice (Figure A4). Reduced mitral valve deceleration time implies that the 
LV is stiffer, and these two echo parameters being reduced could indicate restrictive filling in the T16I KI mice.
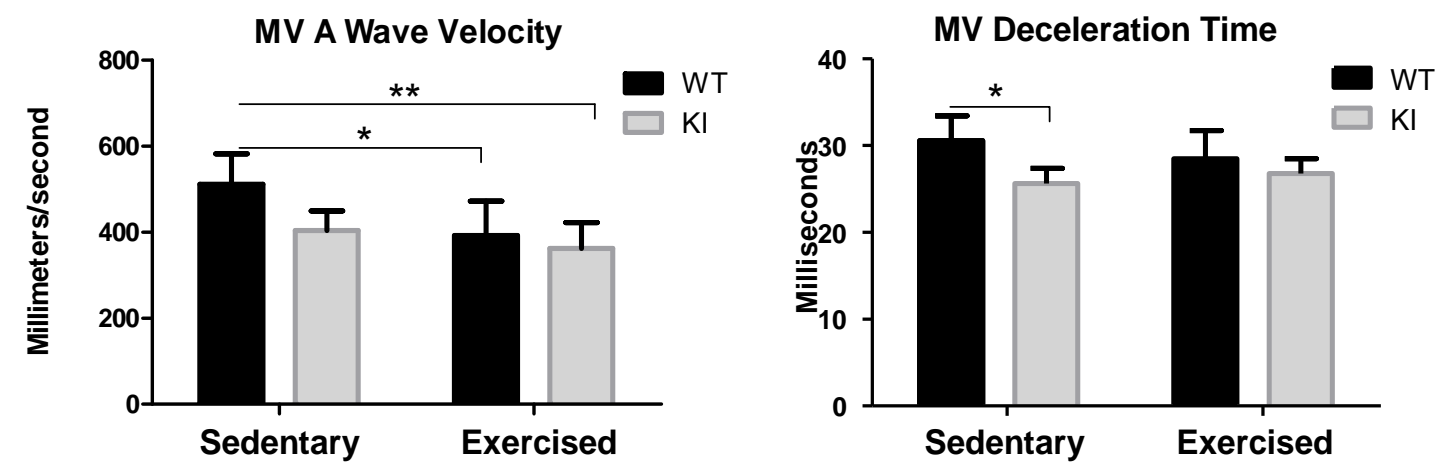

Figure A4: Left-The velocity of the mitral valve A wave is reduced in WT and T16I KI mice after five weeks of treadmill running compared to sedentary WT mice. T16I KI sedentary mice also have a lower A wave velocity, but this difference is not yet significant. Right-SedentaryT16I KI mice have a shorter mitral valve deceleration time compared to sedentary WT mice, which implies a stiffer left ventricle. Significance was determined using two-way ANOVA and Tukey's post-hoc test. $N=6$ for all groups. $* * p<0.01, * * * p<0.001$.

After echo, the mice were then sacrificed and their hearts were excised. Cross sectional cuts ( $\sim 2 \mathrm{~mm}$ thick) were made through the RV and LV of the heart for histology studies. One cross section slice was fixed in 3\% glutaraldehyde for picrosirius red (PSR) staining, and one slice was snap frozen in OCT for oil red O staining. PSR stains linearized collagen fibers and presents as bright orange/yellow birefringence under polarized light. Increased collagen volume fraction in the heart indicates fibrosis. Oil red $\mathrm{O}$ stains adipose tissue. Since a hallmark of arrhythmogenic cardiomyopathy is myocardial infiltration by fibrous and fat tissue, we wanted to determine if this phenotype was present in the T16I KI mice and if long-term volume overload due to treadmill running exacerbated the phenotype. Figure A5 shows bright collagen fibers after PSR staining. Two-way ANOVA shows no significant difference between the four groups, although the variance due to the interaction factor is nearly significant $(p=0.06)$. This interaction factor is lessened $(\mathrm{p}=0.10)$ when the outlier in the exercised WT dataset is removed. It is unclear 
why exercise tends to increase fibrosis in WT mice and decrease fibrosis in the T16I KI mice, which was unexpected. It should be noted that performing a simple student's t-test on the WT and T16I KI sedentary groups gives a p-value of 0.016 , which would indicate significantly more fibrosis in the sedentary T16I KI animals if not for the necessary inclusion of the exercised datasets.
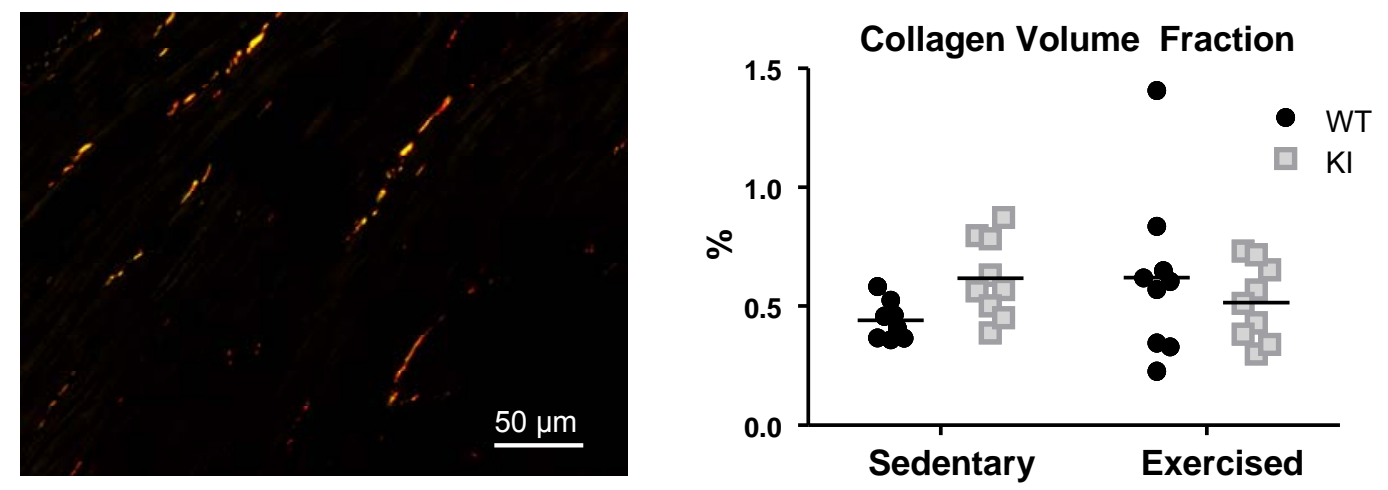

Figure A5: Left-Collagen fibers appear as bright strands upon PSR staining. Right-The collagen volume fraction is determined from the percentage of collagen present in cross sectional slices of the heart. No significance between groups was seen using two-way ANOVA. N=9 for all groups.

Figure A6 shows fat deposits stained with oil red O. There was no significant difference between groups, although exercised WT mice exhibited less fat content while exercised T16I KI mice had more fat relative to the respective sedentary group. The fact that exercise influences the amount of cardiac fat in opposite ways can be seen by the variance due to the interaction factor being significant $(p=0.03)$. Although speculative, it is interesting to suggest that the lower fat percentage in the exercised WT mice compared to sedentary WT mice is the expected, beneficiary physiological response to exercise while the increase in fat in the exercised T16I KI mice compared to sedentary T16I KI mice is indicative of the $\mathrm{AC}$ phenotype beginning to show itself due to exercise-induced volume overload. However, this idea is not supported by the results from PSR staining. 

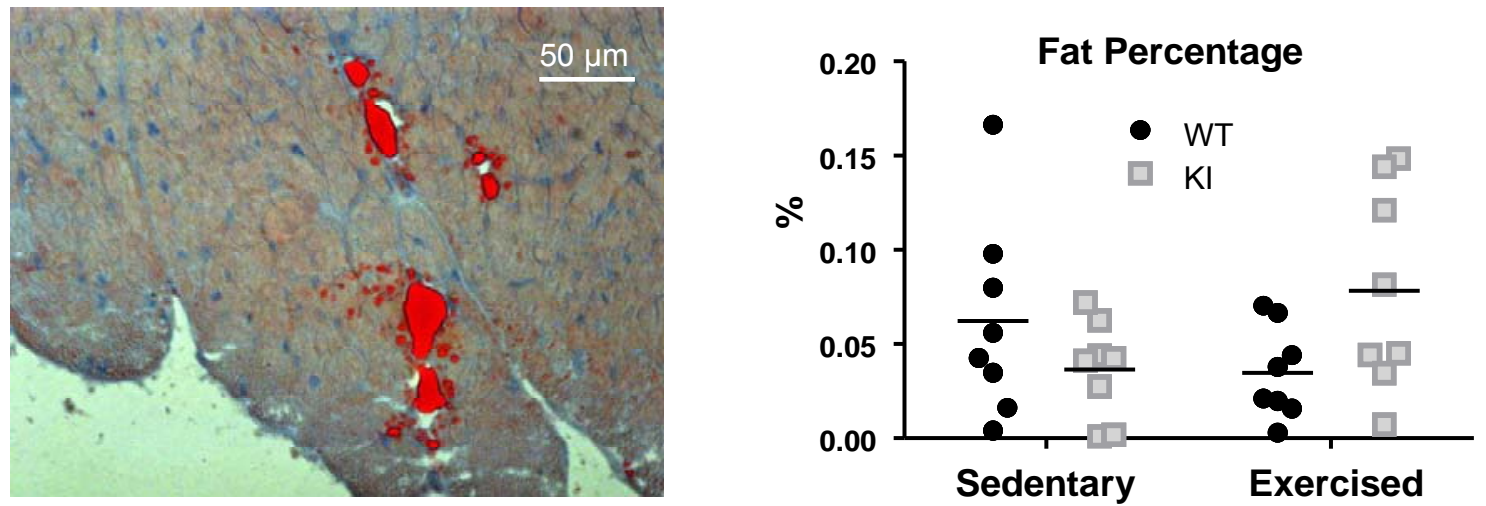

Figure A6: Left-Fat appears bright red after staining with oil red $O$. The percentage of fat was determined by the total area of fat divided by the total cross section of the heart slice. Right-There is no significance amongst the four groups studied, although the variance due to the interaction factor is significant $(p=0.03)$. $N=9$ for all groups.

It is possible that the 10 month old mice used in the treadmill study are too young to show the AC phenotype.

It is thought that the fibrofatty pathology seen in AC patients occurs after myocardial degradation, and apoptosis is one pathway that would lead to a reduction of myocytes in the heart. Therefore, the same fixed heart slices used for PSR staining were also used in a cleaved caspase 3 immunohistochemistry assay to detect apoptotic cells. Cleaved caspase 3 is a central protease in the apoptosis pathway that is often used as an apoptotic marker. Unfortunately, apoptotic cells were not seen in the mouse heart samples from 10 month old mice.

We also performed tissue mechanics on skinned papillary muscles from WT and T16I KI mice to see if the T16I mutation affects passive tension in stretched cardiac muscle. Figure A7 shows the normalized total passive tension, extracellular matrix-based passive tension, and titin-based passive tension as a function of sarcomere length in four month old female mice. At this point ( $\mathrm{n}=6$ for $\mathrm{WT}, \mathrm{n}=5$ for T16I KI) there is no difference between the groups, which is not unexpected. If we assume that every Ig10 
domain is unfolded in the T16I KI mice instead of folded, this would only change passive tension levels by $<1 \%$ between SL $2.0-2.3 \mu \mathrm{m}$ [20]. If instead the presence of the T16I mutation increases titin degradation and reduces the stoichiometry of titin in the sarcomere, this would reduce passive tension, but this does not seem to be the case based on tissue mechanics data and titin gels (T1/MHC ratio is not different between the two genotypes). More tissue mechanics data would strengthen these results.

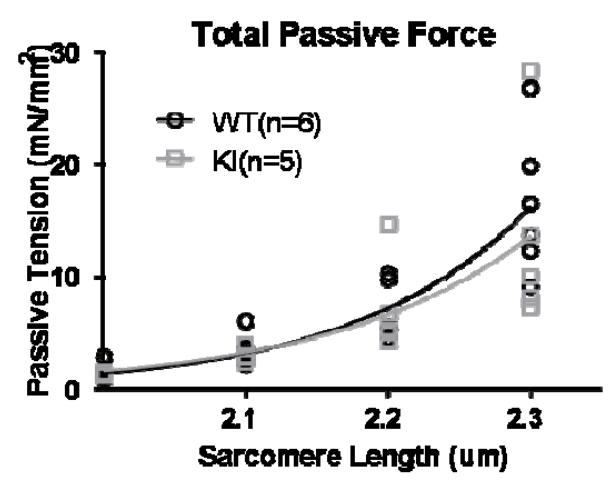

Figure A7: Total, titin, and ECM passive forces in skinned muscle strips from LV papillary muscles. No differences are significant, as determined by using two-way ANOVA and Mann-Whitney rank sum tests. However, the datasets are quite variable.
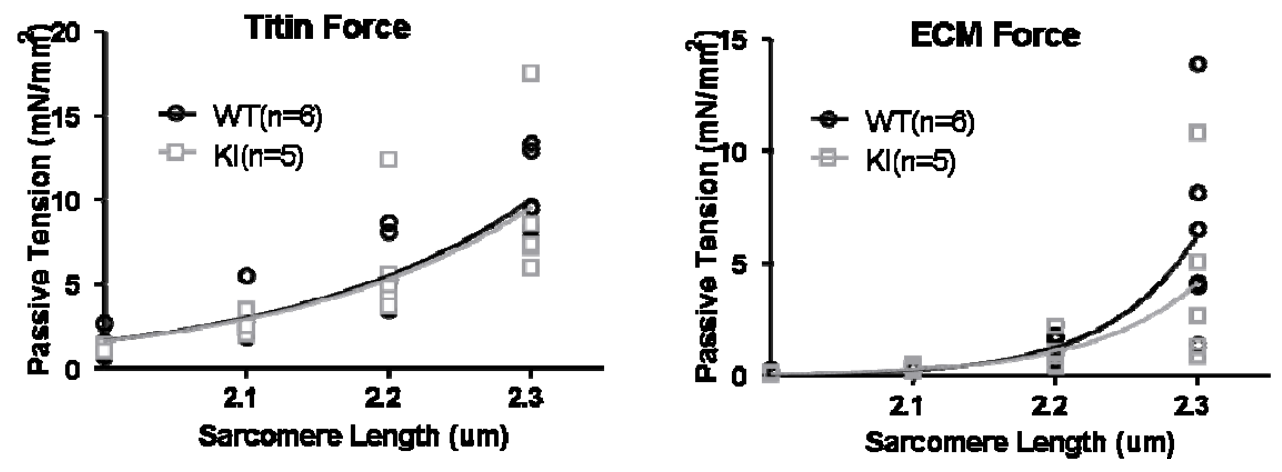

During a stretch-hold protocol on skinned tissue the difference between the peak passive tension and the steady-state passive tension is the viscous stress (i.e. the steady-state force is due entirely to elasticity). Figure A8 shows the viscous stress for WT and T16I KI mice as a function of sarcomere length. No difference was seen between genotypes. 


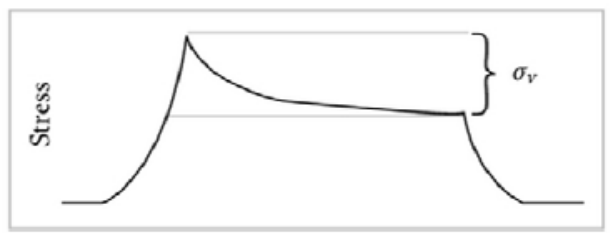

Figure A8: Viscous stress is defined by the difference between peak and steady state stress. No difference was seen between WT and TI6I KI

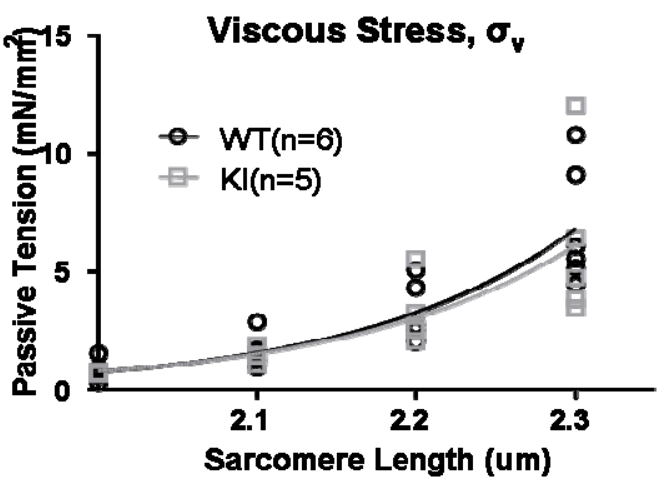

Once steady-state forces are reached a frequency sweep is executed that spans from 0.1 $100 \mathrm{~Hz}$. The amplitudes of the stress trace and strain trace and the phase difference between the two determines the elastic and viscous moduli of the muscle (Figure A9). Quantifying the elastic and viscous moduli gives an overall description of the relative contributions of elastic and viscous sources of passive force. For example, if the stress and strain traces overlap perfectly than a material is purely elastic, while a $90^{\circ}$ phase difference describes purely viscous materials.
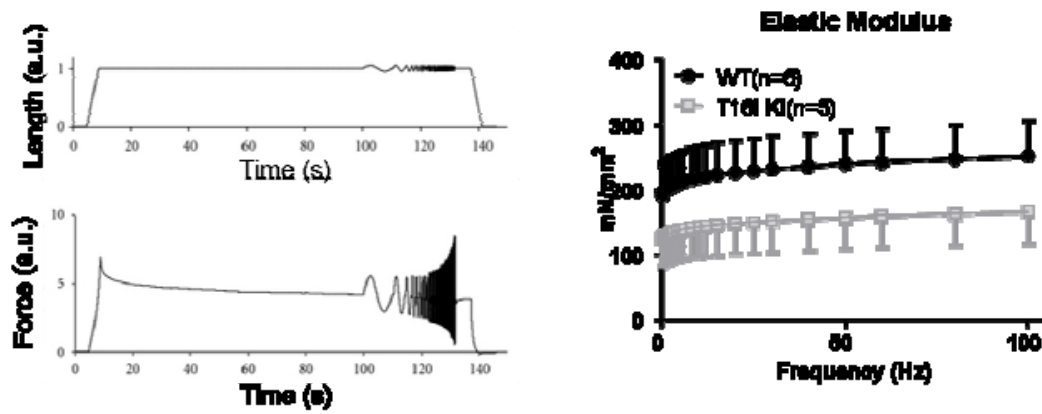

Figure A9: Imposing sinusoidal length changes at various frequencies allows for determination of the elastic and viscous moduli of muscle. Although
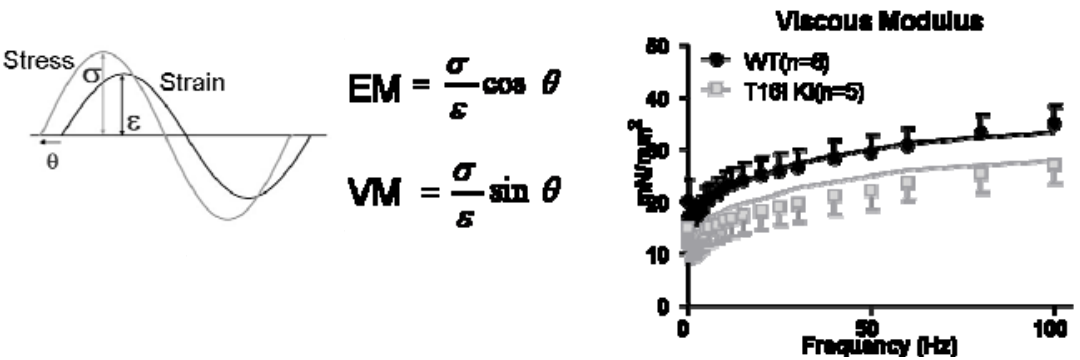
both parameters were higher in the WT tissue, these differences were not significant. 
Future directions

It is not surprising that it has been difficult to actuate the AC phenotype in the T16I KI mice. To begin, the disease often occupies a concealed phase in which the disease pathology is absent. Furthermore, compared to other familial nonischemic cardiomyopathies (such as hypertrophic and dilated cardiomyopathies), individuals with AC present with a wider spectrum of phenotypes, which suggests that other genetic or epigenetic factors are important for determining the severity of the disease [30]. The difficulty in inducing the disease in mice is also evidenced by previous studies that investigated desmosomal mutations that cause AC. For example, in a plakoglobindeficient $\mathrm{AC}$ mouse model the mice did not show right ventricular abnormalities as determined by histology and electron microscopy [43]. Also, an inducible cardiorestricted plakoglobin knockout mouse did not show increased fat deposition, which was attributed to the fact that mouse hearts have much less fat compared to human hearts [44].

It is possible that the AC phenotype will be apparent if older mice are studied. For this reason, we will perform a thorough study on 18 month old WT and T16I KI male mice. The geometry and pump function of these mice will determined via echocardiography, and histology will be performed to determine fat accumulation and fibrosis. We will also perform a cleaved caspase 3 immunohistochemistry assay on the ventricular tissue from these animals to determine if apoptotic cells are present. Any leftover tissue will be quick frozen for future gel analysis and Western Blots.

A novel way that we can look at the effect of the T16I mutation on titin degradation is by performing single myofibril mechanics on WT and T16I KI myofibrils. Myofibrils are subcellular organelles that are composed entirely of serially-linked 
sarcomeres. Studying the mechanics of isolated myofibrils is ideal because there is no tensile contribution from the extracellular matrix and the diffusion distance from the periphery of the myofibril to its center is very short, which allows for measurements of fast kinetics. The goal of this experiment will be to directly measure force-dependent degradation of titin. Our setup allows us to tether a single myofibril (or small myofibril bundle) between a glass needle and cantilever. Length changes can be imposed on the myofibril via a piezotube that moves the position of the glass needle. As the myofibril is stretched, the cantilever displaces and force can be recorded using a focused laser and a quadrant photodetector (very similar to the optical lever system utilized by AFM systems). Then, at different myofibril strains (i.e. different forces borne by titin's elastic I-band region) we will introduce a protease rich solution into the myofibril environment. The proteases will then cleave titin at its most vulnerable locations, with titin degradation inferred by a decrease in titin-based passive tension. If we see a difference in the rate at which titin is degraded between myofibrils from WT and T16I KI mice, we will have direct evidence that the T16I mutation leads to increased titin degradation at the level of the sarcomere.

These proposed experiments are novel and exciting and will hopefully lead to conclusive evidence that the T16I KI mice develop the AC phenotype. This project is worth investigating further because it has the potential to make key discoveries in linking titin to arrhythmogenic cardiomyopathy. Titin is the first sarcomeric protein to be linked to $\mathrm{AC}$ and therefore much can be learned about the disease itself and the myriad roles that titin plays in cardiac myocytes. 


\section{References}

1. Horowits, R. and R.J. Podolsky, The positional stability of thick filaments in activated skeletal muscle depends on sarcomere length: evidence for the role of titin filaments. J Cell Biol, 1987. 105(5): p. 2217-23.

2. Horowits, R., et al., A physiological role for titin and nebulin in skeletal muscle. Nature, 1986. 323(6084): p. 160-4.

3. Wu, Y., et al., Changes in titin isoform expression in pacing-induced cardiac failure give rise to increased passive muscle stiffness. Circulation, 2002. 106(11): p. 1384-9.

4. Warren, C.M., et al., Titin isoform expression in normal and hypertensive myocardium. Cardiovasc Res, 2003. 59(1): p. 86-94.

5. Neagoe, C., et al., Titin isoform switch in ischemic human heart disease. Circulation, 2002. 106(11): p. 1333-41.

6. Yamasaki, R., et al., Protein kinase A phosphorylates titin's cardiac-specific N2B domain and reduces passive tension in rat cardiac myocytes. Circ Res, 2002. 90(11): p. 1181-8.

7. Hidalgo, C., et al., PKC phosphorylation of titin's PEVK element: a novel and conserved pathway for modulating myocardial stiffness. Circ Res, 2009. 105(7): p. 631-8, $17 \mathrm{p}$ following 638.

8. Gutierrez-Cruz, G., A.H. Van Heerden, and K. Wang, Modular motif, structural folds and affinity profiles of the PEVK segment of human fetal skeletal muscle titin. J Biol Chem, 2001. 276(10): p. 7442-9.

9. Ma, K., L. Kan, and K. Wang, Polyproline II helix is a key structural motif of the elastic PEVK segment of titin. Biochemistry, 2001. 40(12): p. 3427-38.

10. Williamson, M.P., The structure and function of proline-rich regions in proteins. Biochem J, 1994. 297 ( Pt 2): p. 249-60.

11. Marko, J.F. and E.D. Siggia, Stretching DNA. Macromolecules, 1995. 28: p. 8759-8770.

12. Cole, C., J.D. Barber, and G.J. Barton, The Jpred 3 secondary structure prediction server. Nucleic Acids Res, 2008. 36(Web Server issue): p. W197-201.

13. Chenna, R., et al., Multiple sequence alignment with the Clustal series of programs. Nucleic Acids Res, 2003. 31(13): p. 3497-500.

14. Dubois, L. and R. Nuzzo, Synthesis, Structure, and Properties of Model Organic Surfaces. Annu Rev Phys Chem, 1992. 43: p. 437-463.

15. Watanabe, K., et al., Different molecular mechanics displayed by titin's constitutively and differentially expressed tandem Ig segments. Journal of Structural Biology, 2002. 137(1-2): p. 248-258.

16. Li, H., et al., Atomic force microscopy reveals the mechanical design of a modular protein. Proc Natl Acad Sci U S A, 2000. 97(12): p. 6527-31.

17. Li, H., et al., Point mutations alter the mechanical stability of immunoglobulin modules. Nat Struct Biol, 2000. 7(12): p. 1117-20.

18. Zhmurov, A., R.I. Dima, and V. Barsegov, Order statistics theory of unfolding of multimeric proteins. Biophys J, 2010. 99(6): p. 1959-68. 
19. Watanabe, K., et al., Different molecular mechanics displayed by titin's constitutively and differentially expressed tandem Ig segments. J Struct Biol, 2002. 137(1-2): p. 248-58.

20. Anderson, B.R., et al., Single Molecule Force Spectroscopy on Titin Implicates Immunoglobulin Domain Stability as a Cardiac Disease Mechanism. Journal of Biological Chemistry, 2013. 288(8): p. 5303-5315.

21. Bell, G.I., Models for the specific adhesion of cells to cells. Science, 1978. 200(4342): p. 618-27.

22. Watanabe, K., et al., Molecular mechanics of cardiac titin's PEVK and N2B spring elements. J Biol Chem, 2002. 277(13): p. 11549-58.

23. Radke, M.H., et al., Targeted deletion of titin N2B region leads to diastolic dysfunction and cardiac atrophy. Proc Natl Acad Sci U S A, 2007. 104(9): p. 3444-9.

24. Moroz, J.D. and P. Nelson, Torsional directed walks, entropic elasticity, and DNA twist stiffness. Proc Natl Acad Sci U S A, 1997. 94(26): p. 14418-22.

25. Irving, T., et al., Thick-filament strain and interfilament spacing in passive muscle: effect of titin-based passive tension. Biophys J, 2011. 100(6): p. 1499508.

26. Rief, M., et al., Reversible unfolding of individual titin immunoglobulin domains by AFM. Science, 1997. 276(5315): p. 1109-12.

27. Saffitz, J.E., A. Asimaki, and H. Huang, Arrhythmogenic right ventricular cardiomyopathy: new insights into disease mechanisms and diagnosis. J Investig Med, 2009. 57(8): p. 861-4.

28. Dennis, P.P., et al., Varying rate of $R N A$ chain elongation during rrn transcription in Escherichia coli. J Bacteriol, 2009. 191(11): p. 3740-6.

29. Dennis, P.P. and H. Bremer, Differential rate of ribosomal protein synthesis in Escherichia coli B/r. J Mol Biol, 1974. 84(3): p. 407-22.

30. Saffitz, J.E., The pathobiology of arrhythmogenic cardiomyopathy. Annu Rev Pathol, 2011. 6: p. 299-321.

31. Herren, T., P.A. Gerber, and F. Duru, Arrhythmogenic right ventricular cardiomyopathy/dysplasia: a not so rare "disease of the desmosome" with multiple clinical presentations. Clin Res Cardiol, 2009. 98(3): p. 141-58.

32. Dudko, O.K., G. Hummer, and A. Szabo, Intrinsic rates and activation free energies from single-molecule pulling experiments. Phys Rev Lett, 2006. 96(10): p. 108101.

33. Dudko, O.K., G. Hummer, and A. Szabo, Theory, analysis, and interpretation of single-molecule force spectroscopy experiments. Proc Natl Acad Sci U S A, 2008. 105(41): p. 15755-60.

34. Raible, M., et al., Theoretical analysis of dynamic force spectroscopy experiments on ligand-receptor complexes. J Biotechnol, 2004. 112(1-2): p. 13-23.

35. Bennett, P.M., et al., The transitional junction: A new functional subcellular domain at the intercalated disc. Molecular Biology of the Cell, 2006. 17(4): p. 2091-2100.

36. Herman, D.S., et al., Truncations of titin causing dilated cardiomyopathy. N Engl J Med, 2012. 366(7): p. 619-28. 
37. Taylor, M., et al., Genetic Variation in Titin in Arrhythmogenic Right Ventricular Cardiomyopathy-Overlap Syndromes. Circulation, 2011. 124(8): p. 876-885.

38. Perrino, C., et al., Cardiovascular effects of treadmill exercise in physiological and pathological preclinical settings. Am J Physiol Heart Circ Physiol, 2011. 300(6): p. H1983-9.

39. Gardin, J.M., et al., Recommendations for a standardized report for adult transthoracic echocardiography: A report from the American society of Echocardiography's nomenclature and standards committee and task force for a standardized Echocardiography report. Journal of the American Society of Echocardiography, 2002. 15(3): p. 275-290.

40. Bang, M.L., et al., The complete gene sequence of titin, expression of an unusual approximately 700-kDa titin isoform, and its interaction with obscurin identify a novel Z-line to I-band linking system. Circ Res, 2001. 89(11): p. 1065-72.

41. Warren, C.M., P.R. Krzesinski, and M.L. Greaser, Vertical agarose gel electrophoresis and electroblotting of high-molecular-weight proteins. Electrophoresis, 2003. 24(11): p. 1695-1702.

42. Sen-Chowdhry, S., et al., Arrhythmogenic Cardiomyopathy: Etiology, Diagnosis, and Treatment. Annual Review of Medicine, 2010. 61: p. 233-253.

43. Kirchhof, P., et al., Age- and training-dependent development of arrhythmogenic right ventricular cardiomyopathy in heterozygous plakoglobin-deficient mice. Circulation, 2006. 114(17): p. 1799-806.

44. Li, J., et al., Cardiac tissue-restricted deletion of plakoglobin results in progressive cardiomyopathy and activation of \{beta\}-catenin signaling. Mol Cell Biol, 2011. 31(6): p. 1134-44. 


\section{Appendix A}

Brian R. Anderson, Julius Bogomolovas, Siegfried Labeit, and Henk Granzier, Single Molecule Force Spectroscopy on Titin Implicates Immunoglobulin Domain Stability as a Cardiac Disease Mechanism. Journal of Biological Chemistry, 2013. 288(8): p. 5303-5315. 


\title{
Single Molecule Force Spectroscopy on Titin Implicates Immunoglobulin Domain Stability as a Cardiac Disease Mechanism*
}

\author{
Received for publication, July 16, 2012, and in revised form, December 10, 2012 Published, JBC Papers in Press, January 6, 2013, DOI 10.1074/jbc.M112.401372 \\ Brian R. Anderson ${ }^{\neq 5}$, Julius Bogomolovas", Siegfried Labeit", and Henk Granzier ${ }^{51}$ \\ From the ${ }^{\ddagger}$ Department of Physics and the ${ }^{5}$ Department of Physiology and Sarver Molecular Cardiovascular Research Program, \\ University of Arizona, Tucson, Arizon 85724 and the "Institute for Integrative Pathophysiology, Universitätsmedizin Mannheim, \\ 68167 Mannheim, Germany
}

Background: A mutation found in titin has been linked to arrhythmogenic cardiomyopathy (AC).

Results: The mutation increases Ig10 instability and susceptibility to degradation.

Conclusion: The mutation compromises the local structure of Ig10 and has a significant effect on Ig10 unfolding dynamics. Significance: Titin is the first sarcomeric protein to be implicated in AC pathology; a novel titin-based disease mechanism is suggested.

Titin plays crucial roles in sarcomere organization and cardiac elasticity by acting as an intrasarcomeric molecular spring. A mutation in the tenth Ig-like domain of titin's spring region is associated with arrhythmogenic cardiomyopathy, a disease characterized by ventricular arrhythmias leading to cardiac arrest and sudden death. Titin is the first sarcomeric protein linked to arrhythmogenic cardiomyopathy. To characterize the disease mechanism, we have used atomic force microscopy to directly measure the effects that the disease-linked point mutation (T16I) has on the mechanical and kinetic stability of Ig10 at the single molecule level. The mutation decreases the force needed to unfold Ig10 and increases its rate of unfolding 4-fold. We also found that T16I Ig10 is more prone to degradation, presumably due to compromised local protein structure. Overall, the disease-linked mutation weakens the structural integrity of titin's Ig10 domain and suggests an Ig domain disease mechanism.

Titin is a giant filamentous protein that spans the entire half sarcomere from the Z-disk to the $\mathrm{M}$-band and is responsible for passive elasticity and the structural integrity of cardiac muscle. Titin is also involved in various cell signaling pathways (1), and mutations in titin have been implicated in numerous diseases, including dilated and hypertrophic cardiomyopathies $(2,3)$. Recently, a mutation in titin was found in a family affected with arrhythmogenic cardiomyopathy $(\mathrm{AC})^{2}(4)$. AC is a primary

\footnotetext{
* This work was supported, in whole or in part, by National Institutes of Health Training Grant GM084905 and Grant HL062881 (to H. G.). This work was also supported by American Heart Association Grant 11PRE7370083 (to B. A.) and grants from European Union FP7 SarcoSi, the DZHK (German Center for Cardiovascular Research), and the BMBF (German Ministry of Education and Research) (to J. B. and S. L.).

${ }^{1}$ To whom correspondence should be addressed: Dept. of Physiology and Sarver Molecular Cardiovascular Research Program, University of Arizona, MRB 340, 1656 E Mabel St., Tucson, AZ 85724-5217. Tel.: 520-626-3641; Fax: 520-626-7600; E-mail: granzier@email.arizona.edu.

${ }^{2}$ The abbreviations used are: AC, arrhythmogenic cardiomyopathy; AFM, atomic force microscopy; $c d f$, cumulative distribution function; MLE, maximum likelihood estimation; SL, sarcomere length; BES, 2-[bis(2-hydroxy-
}

heart muscle disorder characterized by breakdown of healthy cardiac myocytes and replacement with fibrofatty tissue and has been linked with various desmosomal mutations (5-9). AC is associated with severe ventricular arrhythmias and is the leading cause of sudden cardiac death in people under 35 years old (10).

Titin is connected near the Z-disk to actin-based thin filaments and in the A-band to myosin-based thick filaments and also contains an elastic region that is stretched during diastole when the heart ventricles fill with blood. The coupling of titin extension and ventricular stiffness has been extensively studied, and changes in titin-based stiffness accompany diastolic dysfunction (11-13). Titin's extensible I-band region is not bound to actin or myosin and contains three distinct springlike elements (the PEVK element, N2B element, and tandem immunoglobulin (Ig)-like domains (14)) that bear force during sarcomere stretch. The recently discovered AC-linked titin mutation (4) is found in the tenth Ig domain (Ig10) of titin's I-band region. It is the first I-band Ig mutation to be linked to cardiac disease. Ig domains have a stable $\beta$-barrel conformation (15) and are serially connected by short peptide linkers $(16,17)$. When sarcomeres stretch during diastole, the tandem Ig segments in titin's I-band are the first spring element to become taut (18), although they are thought to remain folded under physiological forces $(<5 \mathrm{pN} /$ molecule) and may act as a force buffer under extreme loads.

This study investigates if the AC-linked point mutation affects the structure and kinetics of Ig10 using atomic force microscopy (AFM) and biochemical assays. With AFM, global unfolding of a protein can be monitored, and direct measurements of refolding rates and force-dependent unfolding rates can be made, both of which are crucial for understanding protein dynamics. Biochemical assays allow the study of protein degradation under various proteolytic conditions. The diseaselinked mutation is a $\mathrm{C} \rightarrow \mathrm{T}$ nucleotide transition found in exon

ethyl)amino]ethanesulfonic acid; $\mathrm{pN}$ and $\mathrm{mN}$, pico- and millinewton(s), respectively. 


\section{Ig10 Mutation and Domain Instability}

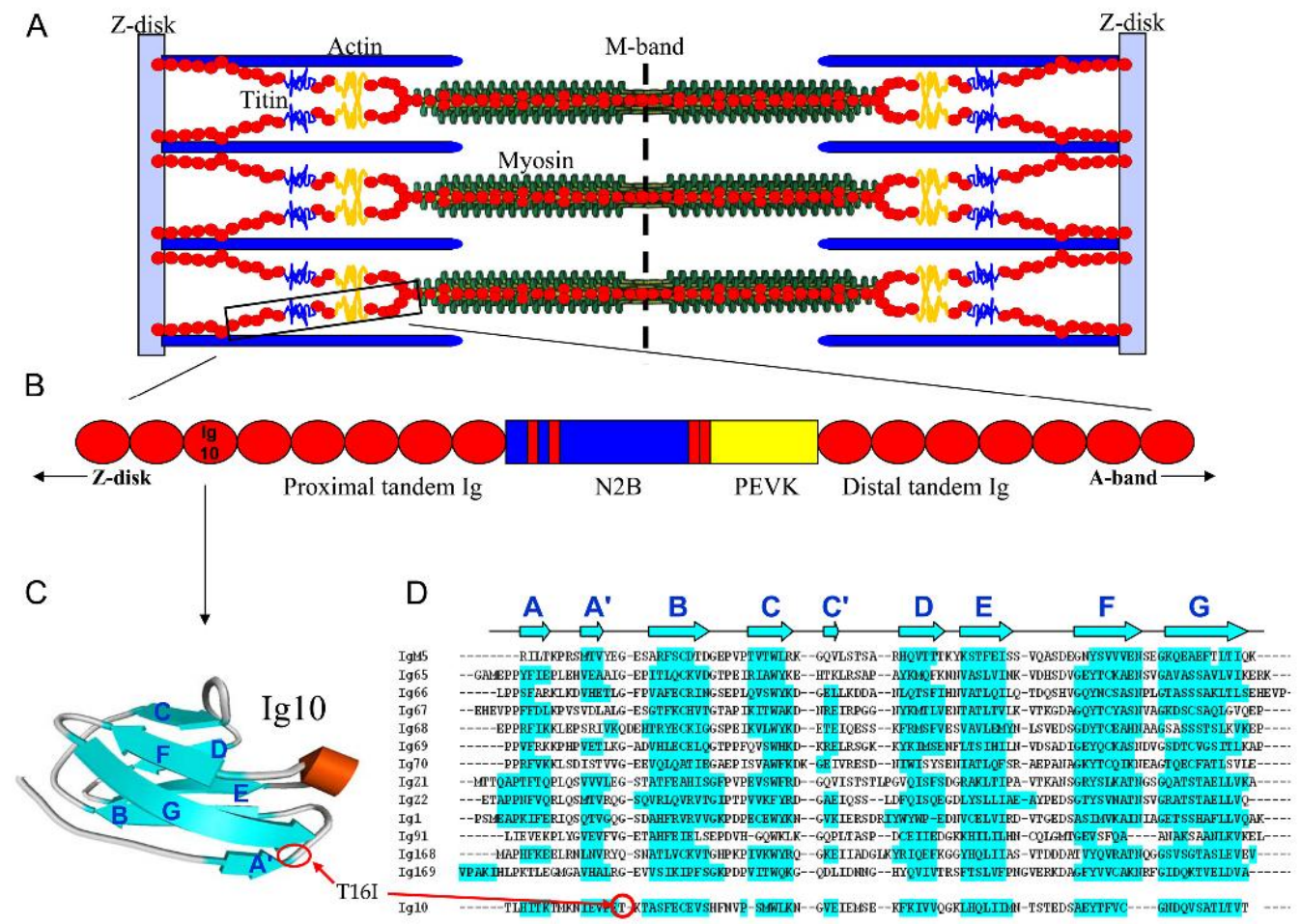

FIGURE 1. A, schematic of a cardiac sarcomere. A single titin molecule spans from the Z-disk to the M-band and contains a large elastic I-band region consisting of tandem Ig domains, the N2B element, and PEVK sequence. B, schematic of part of titin's I-band region. The AC-linked point mutation is in the tenth proximal Ig domain. Note that the 15 proximal and 22 distal Ig domains of the N2B titin isoform are not all shown. $C$, Ig 10 tertiary structure predicted from homology modeling (19). D, alignment of predicted Ig10 secondary structure (20) with solved titin Ig domains shows that the T16l mutation resides in the $\mathrm{A}^{\prime} \mathrm{B}$ peptide loop (40). Residues highlighted in blue have $\beta$-strand secondary structure (predicted in Ig 10 ).

37 of the titin gene (4). Exon 37 encodes for the tenth Ig domain in the proximal tandem Ig segment of the I-band, and the mutation changes the native threonine residue into isoleucine. This mutation is referred to as T16I (threonine is the 16th residue from the $\mathrm{N}$ terminus of Ig10). From homology modeling and secondary structure prediction $(19,20), \operatorname{Ig} 10$ is expected to form an Ig-I $\beta$-barrel structure (15), with the native Thr residue located between the $\mathrm{A}^{\prime}$ and $\mathrm{B} \beta$-strands (Fig. 1). Previous single molecule force spectroscopy and molecular dynamics studies have shown that residues in the $\mathrm{A}^{\prime} \mathrm{B}$ loop are crucial for determining the structural stability of Ig domains via non-covalent interactions with residues in the G $\beta$-strand (21-22), with A mutations either increasing or decreasing the force needed to unfold the domain (23). Although many studies have investigated Ig domain unfolding (23-26), this is the first time a naturally occurring Ig mutation has been studied using AFM and the first time an Ig domain in titin's elastic I-band region has been linked to cardiac dysfunction.

We hypothesize that the T16I mutation alters the conformation of Ig10 and increases its propensity to exist in a non-native, unfolded state. T16I Ig10 may then be susceptible to protease cleavage, which would completely abolish titin's force generation mechanism and would probably lead to pathological remodeling (27). A critical first step toward elucidating the relationship between mutated Ig10 and arrhythmogenic cardiomyopathies is determination of the functional effect of the T16I mutation at the single molecule level.

\section{EXPERIMENTAL PROCEDURES}

Protein Engineering-Two Ig10 pentamers (five identical Ig10 domains linked in series) were created: Ig10 wild type and Ig10 mutant. The mutant 5-mer contains the single AC-linked amino acid mutation (T16I) in all five Ig domains. To best mimic the native rotational constraints on Ig10, the linker sequence between Ig10 domains in the WT and T16I pentamers was chosen such that each Ig10 domain was flanked by the native residue at each terminus (Pro at the $\mathrm{C}$ terminus and Glu at the $\mathrm{N}$ terminus). This resulted in an $\operatorname{Ig} 10(\mathrm{PE}(\operatorname{Ig} 10))_{4}$ construct. We also expressed a naturally occurring fragment containing seven Ig domains (Ig7-13) and a mutant version of this heteropolyprotein in which the only difference is the T16I mutation in Ig10. Synthetic DNA coding for five human Ig10 domains connected in series and Ig7-13 was purchased from Geneart (Regensburg, Germany), inserted into vectors, and expressed in Escherichia coli. All constructs were inserted into pETM11 vectors except for Ig7-13 WT, which was inserted in

ASEMTE VOLUME $288 \cdot$ NUMBER $8 \cdot$ FEBRUARY 22,2013 


\section{Ig10 Mutation and Domain Instability}

pET9D for technical reasons. Protein expression and purification were performed using standard methods (28).

Single Molecule Force Spectroscopy-The mechanical and kinetic properties of purified Ig10 5-mers were probed with an MFP3D atomic force microscope from Asylum Research (Santa Barbara, CA). Freshly thawed recombinant protein in buffer $(25$ mм BES, 2.5 mм EGTA, 1.5 mм $\mathrm{MgCl}_{2}, 1.25 \mathrm{~mm} \mathrm{NaATP}, 165$ $\mathrm{mm} \mathrm{KCl}, \mathrm{pH} 7.0$ ) was spotted on gold-coated microscope slides at $\sim 5-10 \mu \mathrm{g} / \mathrm{ml}$ and incubated at $4{ }^{\circ} \mathrm{C}$ for at least $1 \mathrm{~h}$. The Ig10 5 -mers were engineered with two $\mathrm{C}$-terminal cysteines to allow thiol-gold bonds to anchor the protein to the slide surface. Few functional groups besides thiols bind strongly to gold (29), so exploiting the thiol-gold bond encourages the protein's short $\mathrm{C}$-terminal extension to attach to the slide surface while discouraging nonspecific binding between the globular Ig domains and gold slide. Unbound molecules were removed from the slide through pipette rinses with buffer. Surface protein density was kept low to minimize protein-protein interactions and the probability of multiple molecules attaching to the atomic force microscope tip simultaneously.

With one end of the polyprotein attached to the slide surface, a piezo-controlled silicon nitride cantilever tip (MLCT, Veeco Probes, Plainview, NY) was driven toward the protein-coated surface from above and nonspecifically adsorbed protein. The end-to-end distance of the Ig105-mer was constrained between the slide and the cantilever tip, and the molecule was stretched along this axis as the cantilever retracted from the slide surface at $1000 \mathrm{~nm} / \mathrm{s}$. This constant velocity stretching generates a force versus displacement curve that characterizes the elastic and mechanical properties of the tethered protein. The displacement of the cantilever base was determined directly via an integrated linear voltage differential transformer. To determine the force exerted on the molecule as the tip was pulled away from the surface, Hooke's law $(F=-k x)$ was used, where $F(x)$ is the force needed to extend the molecule a given distance, $x$ is the distance away from equilibrium the cantilever is bent, and $k$ is the spring constant of the cantilever. Cantilever stiffness was established by measuring its mean thermally driven vertical displacement $(x)$ and then applying the equipartition theorem $k\left\langle x^{2}\right\rangle=k_{B} T$, where $k_{B}$ is Boltzmann's constant and $T$ is absolute temperature. Stiffness is typically $20-25 \mathrm{pN} / \mathrm{nm}$. To accurately measure the force-displacement relationship of the Ig pentamer, the end-to-end length of the tethered molecule was corrected for cantilever bending.

As a tethered Ig pentamer is stretched, force develops in the molecule until a single Ig10 unfolding event occurs. When an Ig domain unravels from a native, compact state to a denatured, extended state, the contour length of the entire 5-mer is increased by $\sim 28 \mathrm{~nm}$ (the diameter of a folded Ig domain is $\sim 5$ $\mathrm{nm}$, and the backbone contour length of the unfolded Ig10 polypeptide is 87 amino acids $\times 0.38 \mathrm{~nm} / \mathrm{amino}$ acid $=33 \mathrm{~nm}$ ) This sudden increase in contour length following a high force unfolding event results in an identifiable "force peak." This process of polyprotein extension and unfolding continues until all serially linked Ig domains have unfolded or until the protein is displaced from the cantilever tip. Because the thiol-gold bond approaches covalent strengths ( $\sim 44 \mathrm{kcal} / \mathrm{mol})(29)$, the molecule is much more likely to be displaced from the cantilever tip than from the slide surface. These successive unfolding events generate a characteristic sawtooth pattern; this "molecular fingerprint" is used to determine when a single Ig10 5-mer is being stretched. If more than five low force peaks are present or if consecutive force peaks are not separated by $\sim 28 \mathrm{~nm}$ periodicity, the tip has attached to multiple pentamers at once; these data are not analyzed.

Stretch-relaxation-restretch was also implemented to directly measure the refolding rate of WT and T16I Ig10. A standard force-extension trace was overridden by a secondary trigger that initialized a user-defined drive wave that controls piezoelectric position. For the stretch-relaxationrestretch protocol, the number of unfolded domains that were allowed to refold was limited by the molecular extension during the low force hold. For example, in Fig. 6, the molecule was relaxed to the middle of the first Ig10 unfolding peak at $\sim 25 \mathrm{~nm}$ extension (the first blue peak is adhesion between the cantilever tip and slide surface). Although there are four unfolding peaks in the initial extension, only three domains can possibly refold during the hold time (which is at an extension of $\sim 25-30 \mathrm{~nm}$ ) because the fourth refolding event would require $\sim 50 \mathrm{pN}$ of bend in the cantilever tip (i.e. the force needed to extend four folded domains to $25-30 \mathrm{~nm}$ during the initial stretch). The energy required to bend a cantilever tip $(k=20 \mathrm{pN} / \mathrm{nm})$ by this amount is $63 \mathrm{pN} \cdot \mathrm{nm}$, which is 15 times larger than $k_{B} T$; therefore, the fourth unfolded Ig domain is prohibited from refolding.

Force clamp AFM protocols were also performed to measure force-dependent unfolding. The force clamp protocol uses a feedback mechanism to maintain tension in a tethered 5-mer following Ig unfolding. For each unfolding event, we measured the time interval $\Delta t_{i}$ between the start of the force clamp and the unfolding event. It takes tens of milliseconds for the feedback to reestablish the force clamp, and this time was subtracted from the raw time data to correct for the amount of time that the 5-mer is not held at the clamp force. For two-step unfolding, $\Delta t_{i}$ was taken from the start of the force clamp to the time of the second unfolding transition. Because actual hold forces varied slightly between tethered molecules, we analyzed data in $5 \mathrm{pN}$ bins (i.e. data from clamps between 53 and $57 \mathrm{pN}$ were analyzed together and considered as $55 \mathrm{pN}$; root mean square force noise is $\sim 7 \mathrm{pN}$ ).

Removing Unfolding Force Peak Dependence-Although the Ig10 pentamers contain identical domains, average unfolding force increases with peak number (Table 1). To increase the robustness of fits between simulated and experimental data, we removed this peak dependence and thus reduced the spread in our data. Order statistics theory (30) was implemented to generate a parent distribution of unfolding force (describing a single isolated domain) from data gathered from unfolding of Ig 10 pentamers,

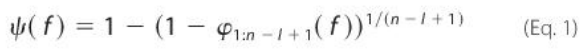

where $\Psi$ represents the parent cumulative distribution function (cdf) and $\varphi$ is the cumulative distribution function of the $l$ th unfolding force peaks of a homopolyprotein of length $n$.

Monte Carlo Simulations-Monte Carlo methods were used to simulate AFM force-extension traces. Starting from five seri- 


\section{Ig10 Mutation and Domain Instability}

ally linked Ig domains with a defined unfolding rate at zero force $\left(\alpha_{0}\right)$ and distance along the reaction coordinate from the folded state to the transition state $\left(\Delta x_{u}\right)$, the molecule was stretched at $1000 \mathrm{~nm} / \mathrm{s}$ (mimicking AFM experiments). At each time point, the force acting on the 5-mer was determined from force balance between cantilever bend (set at $22 \mathrm{pN} / \mathrm{nm}$ ) and the force required to stretch the polypeptide (the combination of unfolded and folded domains) using the wormlike chain equation (31). The probability of unfolding is $p=j \cdot \alpha(F) \cdot \Delta t$, where $j$ is the number of folded domains, $\alpha(F)=\alpha_{0} \exp \left(F \cdot \Delta x_{u} /\right.$ $\left.k_{B} T\right)$ is the force-dependent unfolding rate constant, and $\Delta t$ is the time step. A contour length increase of $28 \mathrm{~nm}$ was imposed for each unfolding event, and the persistence length of the polypeptide chain was set to $0.4 \mathrm{~nm}$.

Unfolding Rate Error Estimation-Bootstrap sampling was used to determine uncertainty in the zero force unfolding rate for WT and T16I Ig10. 10,000 bootstrap samples were generated by resampling with replacement from our AFM data. Resampling was performed for each unfolding force peak (i.e. peak $i$ values of a bootstrap data set were taken from peak $i$ experimental forces), and an average parent cdf (with peak dependence removed) was generated for each bootstrap sample. This cdf was then compared with Monte Carlo simulation cdfs to determine the unfolding rate that best describes the bootstrap cdf. The S.D. of the 10,000 bootstrap unfolding rates is reported as the error in unfolding rate for WT and T16I Ig10. The distance to the transition barrier $\left(\Delta x_{u}\right)$ was fixed at $0.3 \mathrm{~nm}$ for bootstrap sampling.

Maximum Likelihood Estimation-All data acquired from the AFM refolding protocol were analyzed together using maximum likelihood estimation (MLE) to determine the refolding rate of WT and T16I Ig10. Although we have evidence for two-step refolding, this process was observed too rarely to generate a data set large enough for analysis. Therefore, partial refolding was considered a 0.5 refolding event for MLE. Domain refolding was modeled as a Bernoulli trial with a success probability of $1-e^{-\beta \cdot t}$. The likelihood function we want to maximize is as follows,

$$
L(\beta \mid \bar{x}, \bar{t})=\Pi\left(1-e^{-\beta \cdot t_{i}^{x}}\right)^{x}\left(e^{-\beta \cdot t_{i}}\right)^{1-x_{i}}
$$

where $\beta$ is the refolding rate, $x$ and $t$ are the measured observables, and the likelihood is the product of all possible refolding events. The vectors $\boldsymbol{x}$ and $\boldsymbol{t}$ are length $N$ (number of domains able to refold), with $x_{i}=1$ if refolding occurs and 0 otherwise, and $t_{i}$ equal to the user-defined hold time. To determine uncertainty in estimated refolding rate, we generated 10,000 bootstrap samples from our experimental data. We then performed MLE on each of the new data sets (to obtain a best fit $\beta^{\prime}$ for each bootstrap sample) and took the S.D. value of all $10,000 \beta^{\prime}$ to determine the error in our refolding rate estimate for our experimental data set.

Proteolysis Assays and Mass Spectrometry-Purified trypsin from bovine pancreas was purchased from Sigma. Freshly dissolved trypsin (50 mm Tris, $\mathrm{pH} 7.6)$ was incubated with Ig10 5 -mer at $37^{\circ} \mathrm{C}$ until the reaction was stopped with reducing buffer. Proteins were separated with SDS-PAGE and Coomassie-stained. Orbitrap Velos (Thermo Fisher) with an Advion Nanomate (Ithaca, NY) source operated in data-dependent
MS/MS mode was used for determining $\mathrm{N}$-terminal peptides of protein degradation products. High resolution mass measurement of peptides was performed by matrix-assisted laser desorption/ionization (MALDI). The MALDI experiments were performed on a Bruker Ultraflex III MALDI TOF-TOF instrument using sinapinic acid as the matrix

Physiological protease degradation assays were performed on Ig7-13 fragments (with or without T16I mutation in Ig10). Fresh mouse hearts were blended in $800 \mu \mathrm{l}$ of $1 \times$ PBS (either $\mathrm{pH} 5$ or 8$)+1.5 \mathrm{~mm} \mathrm{CaCl}_{2}$. The resulting $\mathrm{pH}$ of the heart extract was $\sim 6.3$ and $\sim 7.4$, as determined by a micro- $\mathrm{pH}$ electrode (Lazar Research Laboratories, Los Angeles, CA). The heart extract was then added to WT and mutant Ig7-13. This mixture was incubated at $37^{\circ} \mathrm{C}$, and a portion of the mixture was removed at various time points and spun down at 13,000 $\mathrm{rpm}$ for $2 \mathrm{~min}$. The resulting supernatants were then solubilized and separated using SDS-PAGE.

In Vivo Simulation-Titin-based passive tension (in $\mathrm{mN} / \mathrm{mm}^{2}$ ) as a function of sarcomere length (SL) was determined from muscle mechanics data (32), and the force experienced per titin molecule was calculated from thick filament density and a stoichiometry of six titin molecules per half thick filament (33). The working SL in mice is between 1.8 and 2.2 $\mu \mathrm{m}$ (34), and we assumed a sinusoidal relationship between SL and time, with SL varying between 1.8 and $2.2 \mu \mathrm{m}$ at $1 \mathrm{~Hz}(10$ $\mathrm{Hz}$ showed similar results). From this, we estimated $<5 \mathrm{pN}$ of force acting on each titin molecule at a diastolic SL of $2.2 \mu \mathrm{m}$. Force-dependent unfolding was taken as $\alpha(F)=\alpha_{0} \exp \left(F \cdot \Delta x_{u} /\right.$ $k_{B} T$ ) because the force-dependent unfolding rates we measured experimentally were for force levels much greater than the physiological range.

\section{RESULTS}

Ig10 Unfolding-To determine how the T16I mutation affects the mechanical and kinetic stability of Ig10, mutant and wild type Ig10 5-mers were stretched and unfolded at a pulling speed of $1000 \mathrm{~nm} / \mathrm{s}$. Representative force-extension traces for WT and T16I Ig10 are shown in Fig. 2B. Each of the first five force peaks represents the force needed to unfold an Ig10 domain. Ig10 unfolding results in a contour length increase, which removes the tension from the system and results in the large force drop immediately following a force peak. The last force peak is due to the fully unfolded Ig10 5-mer polypeptide displacing from the cantilever tip. Although each of the first five force peaks is due to unfolding of a single Ig10 domain, unfolding force is peak-dependent, with average unfolding force increasing with peak number (Table 1).

It should also be noted that AFM experiments on WT and mutant Ig7-13 proteins did not show different unfolding forces (data not shown). However, this result was expected because Ig domain unfolding is a stochastic process, and the average unfolding force for each peak in the sawtooth pattern probably contains the unfolding forces of Ig7, Ig8, Ig9, etc., thus masking any real effects.

Ig domain unfolding rate can be described as a force-dependent process by $\alpha(F)=\alpha_{0} \exp \left(F \cdot \Delta x_{u} / k_{B} T\right)$, where $\alpha_{0}$ is the unfolding rate constant at zero force, $F$ is the external applied force, $\Delta x_{u}$ is the unfolding distance along the reaction coordi- 


\section{Ig10 Mutation and Domain Instability}

A

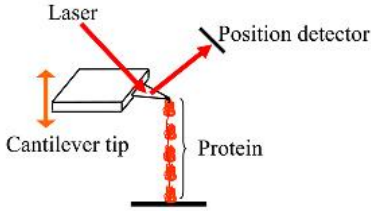

B
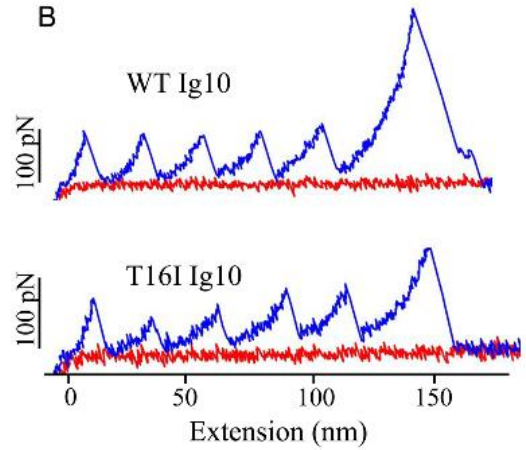

FIGURE 2. $A$, simplified AFM schematic. $B$, representative force-extension traces of WT and T16l Ig 105 -mer stretched at $1000 \mathrm{~nm} / \mathrm{s}$. The red trace indicates the cantilever tip approaching the protein-coated slide surface, and the blue trace indicates the tip moving away from the surface with a tethered protein. The first five low force peaks are due to lg domain unfolding, with an increase in contour length and subsequent decrease in force immediately following each unfolding event. The last force peak is due to the fully unfolded 5-mer being displaced from the cantilever tip.

TABLE 1

Average unfolding force \pm S.E. of each peak for $1000 \mathrm{~nm} / \mathrm{s}$ pulling velocity

Unfolding force is peak-dependent, and T16I results in a lower unfolding force for all peaks. These numbers were generated from 64 WT force-extension traces and 51 T161 traces.

\begin{tabular}{llllll}
\hline & \multicolumn{5}{c}{ Average unfolding force } \\
\cline { 2 - 5 } & Peak 1 & Peak 2 & Peak 3 & Peak 4 & Peak 5 \\
\hline \multicolumn{5}{c}{$p N$} \\
WT & $120 \pm 2$ & $124 \pm 2$ & $128 \pm 2$ & $134 \pm 2$ & $143 \pm 4$ \\
T161 & $92 \pm 2^{a}$ & $97 \pm 3^{a}$ & $106 \pm 2^{a}$ & $117 \pm 2^{a}$ & $126 \pm 4^{b}$ \\
\hline${ }^{a} p<0.001$. \\
${ }^{b} p<0.01$.
\end{tabular}

nate, and $k_{B} T$ is thermal energy at room temperature. A Monte Carlo approach was used to simulate AFM unfolding experiments over hundreds of $\left(\Delta x_{u}, \alpha_{0}\right)$ pairs. Comparing the experimental unfolding force distribution with the simulated force distribution allows estimation of $\alpha_{0}$ and $\Delta x_{u}$ for WT and T16I Ig10. Because unfolding force is peak-dependent, the cdfs of unfolding forces are also peak-dependent, but this peak dependence was removed according to order statistics theory (see "Experimental Procedures"). As a result, the cdf describing the first unfolding event in a collection of force-extension curves now overlaps with the cdf describing the last unfolding event (which, by definition, is the parent cdf; Fig. 3). The transformed cdfs (one for each peak) were then averaged to generate a master parent cdf for either WT or T16I Ig10. This method is preferred because data from all unfolding force peaks are analyzed simultaneously, increasing robustness of fits. Also, it is incorrect to pool unfolding forces from different unfolding force peaks because each peak unfolding force distribution describes a different process (e.g. the force needed to unfold one domain when five folded domains are connected in series is different, on average, from the force needed to unfold one domain when 10 folded domains are connected in series). The same transformations were used on Monte Carlo simulations to generate a master parent cdf for each $\left(\Delta \mathrm{x}_{u}, \alpha_{0}\right)$ pair. The theoretical and experimental cdfs were compared empirically using the Kolmogorov-Smirnov test (35) to find the $\left(\Delta \mathrm{x}_{u}, \alpha_{0}\right)$ pair used in the Monte Carlo simulations that generated the master parent cdf most consistent with the experimental cdf for the WT and T16I Ig10 5-mer simulations. WT Ig10 was best described by $\Delta x_{u}=0.300 \pm 0.025 \mathrm{~nm}$ and $\alpha_{0}=0.004 \pm 0.0005$ $\mathrm{s}^{-1}$, and T16I Ig10 was best described by $\Delta x_{u}=0.300 \pm 0.025$ $\mathrm{nm}$ and $\alpha_{0}=0.018 \pm 0.0029 \mathrm{~s}^{-1}$ ( \pm bootstrap S.D.). This analysis shows that the distance to the transition barrier along the molecular end-to-end length reaction coordinate is unchanged by the AC-linked mutation, whereas the unfolding rate at zero force is increased $\sim 4$-fold.

The previous method gives the unfolding rate at zero force, but we were also interested in force-dependent unfolding rates. Therefore, we probed the effect of the disease-linked mutation on the ability of Ig10 to resist unfolding at different external force levels. Using AFM feedback, we were able to hold tethered proteins at constant force to directly determine force-dependent unfolding rates. For this protocol, we stretched a tethered protein until a set force level developed and then held the protein at that force. This force is held until an Ig domain unfolds, an event that immediately increases the contour length of the molecule and removes the tension from the system. After the Ig rupture, however, the cantilever tip quickly moves further away from the surface until the set force level is reestablished. The molecule is then held at that force until another Ig domain unfolds and the process continues. This method complements standard AFM data by producing information in the time domain (e.g. hold times prior to Ig unfolding) instead of the force domain. Sample force clamp data for WT Ig10 are shown in Fig. 4A. Plotting extension versus time results in a step pattern that has constant extension increases ( $\Delta$ Ext) following Ig unfolding; this pattern also acts as the molecular fingerprint of the Ig10 5-mer. Clamp forces were between 50 and $80 \mathrm{pN}$, and molecular hold time was $5 \mathrm{~s}$. Force clamp and hold time values were chosen to minimize drift effects and increase the chances of seeing unfolding events prior to the molecule detaching from the cantilever tip. Fig. $4 B$ shows the $\Delta$ Ext histograms for WT and T16I Ig10 for all force clamp experiments. Each histogram clearly shows a well defined peak distribution in the addition to a small collection of low $\Delta$ Ext data points. The Gaussian fit to the main peak is centered at $23.8 \mathrm{~nm}$ for the WT distribution and 23.0 for T16I. Although the contour length of the polypeptide increases by $\sim 28 \mathrm{~nm}$ following domain unfolding, the extension increase is less because of polymer coiling. From the wormlike chain equation,

$$
F=\frac{k_{B} T}{L_{p}}\left(\frac{1}{4\left(1-z / L_{C}\right)^{2}}+\frac{z}{L_{C}}-\frac{1}{4}\right)
$$

a contour length gain of $28 \mathrm{~nm}$ would only result in an extension increase of $\sim 22 \mathrm{~nm}$ at a hold force of $60 \mathrm{pN}$ (average clamp, 62.1 


\section{Ig10 Mutation and Domain Instability}
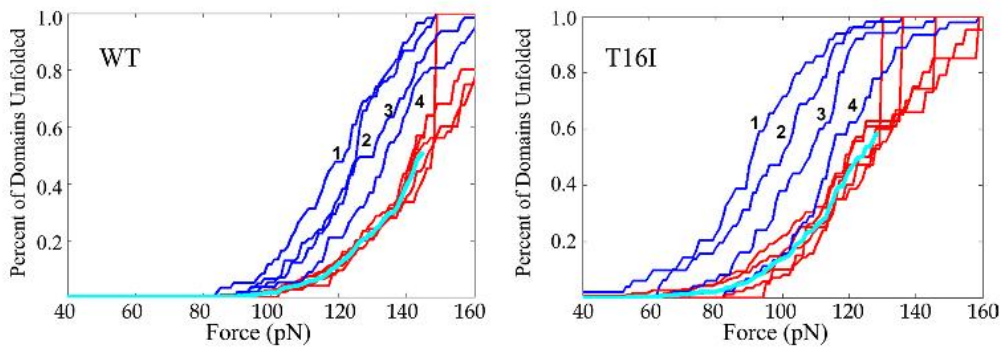

FIGURE 3. cdfs of unfolding forces for WT and T16IIg10 5-mers. For each force value, the $y$ axis value indicates the percentage of domains that unfolded at or below that force. The blue traces are the cdfs of peaks $1-4$. The red traces are the cdfs of peaks 1-4 after transforming to the parent cdf using order statistics (30). The cdf of peak 5 is also in red (because we are stretching a 5 -mer, by definition the cdf of the last unfolding peak forces is the parent cdf). The cyan traces are the average of the five parent cdf (red) traces. These averaged parent cdfs were used to compare experimental data with simulated data (after simulated are the average of the five parent cdf (red) traces. These averaged parent cdfs were used to compare experimental data with simulated data (after simulated
data were similarly processed). This analysis reduces the spread in unfolding force data and allows all peaks to be analyzed together, which increases the data were similarly processed). This analysis
sample size and improves fitting accuracy.

A
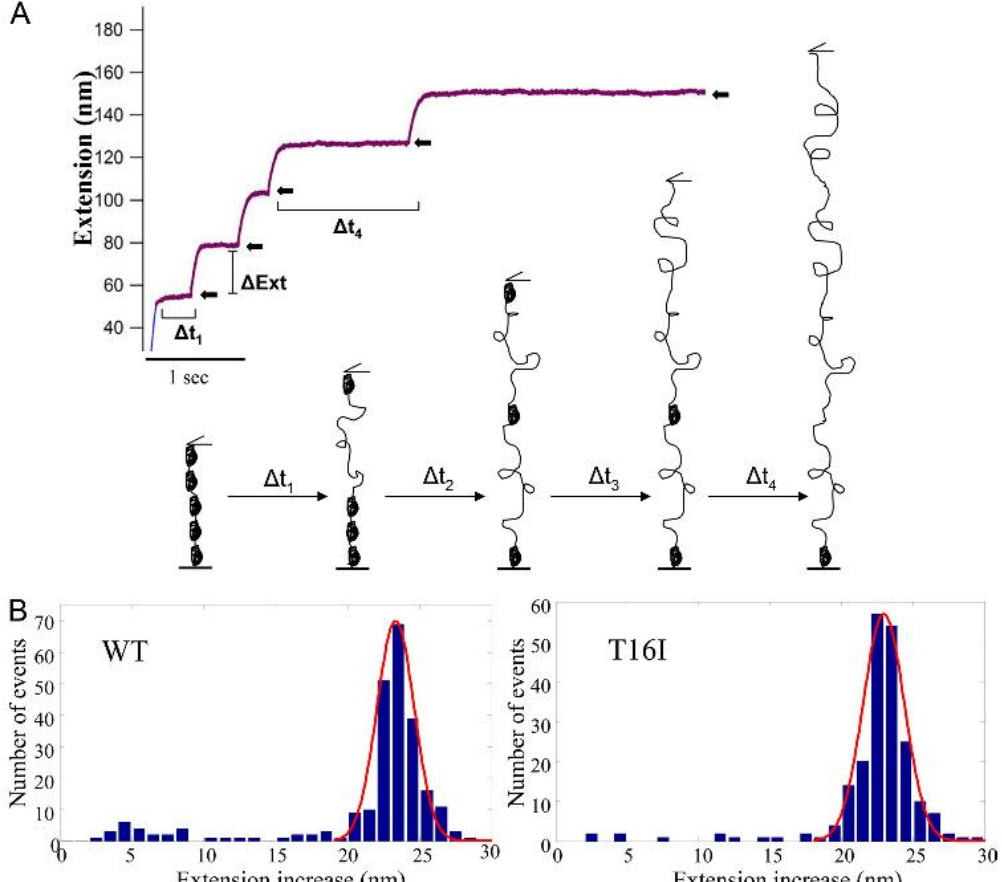

Extension increase (ntm)

Extension increase $(\mathrm{nm})$

FIGURE 4. AFM force clamp protocol. A, tethered protein s were held at constant force for $5 \mathrm{~s}$. After an unfolding event (arrows), the cantilever quickly retracted level was restored, resulting in a stepwise pattern. The extension difference $(\Delta E x t)$ between steps represents the extension increase following an unfolding event. The hold time prior to a domain unfolding is measured from when the residual tension is initially reached (start of the purple trace). For example, the second Ig domain takes $t=\Delta t_{1}+\Delta t_{2}$ to unfold. $B$, histograms of extension increases. The histograms represent the compilation of all force clamp data. The red Gaussian trace is a best fit to the histogram. The smooth distribution is centered at $23.3 \mathrm{~nm}$ for WT lg $10 \mathrm{and} 23.0 \mathrm{~nm}$ for T16l. The data that do not fit the Gaussian distribution represent the individual segments of two-step unfolding events. Two-step unfolding is 5 times more prevalent in WT $\lg 10$.

$\mathrm{pN}$ for WT and $60.6 \mathrm{pN}$ for T16I), which is similar to the Gaussian fit peaks in Fig. $4 B$. The short extension increases that lie outside of the Gaussian fit represent two-step unfolding (i.e. a short extension increase was always partnered with a second, complementary short increase, and these two extensions summed to the length of a singular unfolding event (i.e. a 7-nm increase immediately followed by a 16-nm increase instead of a single 23-nm increase)). It is worth noting that two-step unfold- ing was seen $10 \%$ of the time in WT Ig10 (23 of 221 unfolded domains) but only $2 \%$ of the time in the T16I mutant ( 5 of 204).

Because the tethered polyproteins are under constant force in the AFM force clamp protocol, we were able to determine the force-dependent unfolding rates of WT and T16I Ig10. Fig. 5 shows the empirical cumulative distribution functions of the times at which WT and T16I Ig10 unfold while under $65 \mathrm{pN}$ of external force (step pattern), with the best fit to $1-e^{-\alpha(F) \cdot t}$ 


\section{Ig10 Mutation and Domain Instability}

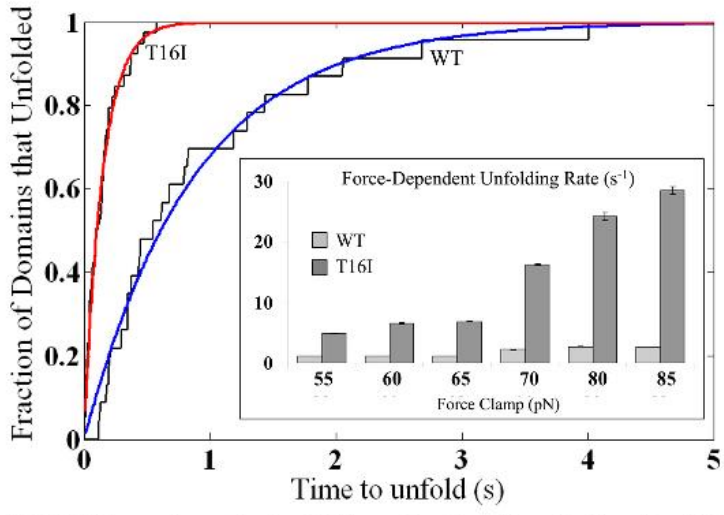

FIGURE 5. Force-dependent unfolding rates. From the collection of unfolding times acquired at a given residual tension, we are able to fit the cumulative distribution function to $1-e^{-\alpha(F) \cdot t}$, where $\alpha(F)$ is the unfolding rate at a given force. The empirical cdfs shown were generated from force clamp valgiven force. The empirical cdfs shown were generated from force clamp val-
ues between 63 and $67 \mathrm{pN}$ (average $\sim 65 \mathrm{pN}$ ). The smooth fits yield $\alpha(F=$ ues between 63 and $67 \mathrm{pN}$ (average $\sim 65 \mathrm{pN})$. The smooth fits yield $\alpha(F=$
$65)=1.135 \mathrm{~s}^{-1}$ for WT $\lg 10$ and $\alpha(F=65)=6.865 \mathrm{~s}^{-1}$ for T16l Ig10. Inset force-dependent unfolding rates for WT and T16l Ig10. Error bars, S.E.

overlaid $\left(R^{2} \sim 0.99\right)$. Note that the collection of unfolding times is well described by a single exponential, which strongly suggests that each domain in the 5-mer unfolds independently. The unfolding rate at $65 \mathrm{pN}$ was found to be $1.135 \mathrm{~s}^{-1}$ for WT Ig10 and $6.865 \mathrm{~s}^{-1}$ for T16I Ig10, a 5 -fold increase. The inset of Fig. 5 shows force-dependent unfolding rates at five clamp forces.

Ig10 Refolding - The unfolding rate is important to determine the susceptibility of a protein to exist in a mechanically denatured state, but the refolding rate is equally important. To determine how the disease-linked mutation affects the refolding rate of Ig10, we implemented a refolding protocol using AFM. Tethered molecules were stretched until most domains had unfolded, a user-defined drive wave was executed that relaxed the tethered molecule and allowed domain refolding events to occur for a set amount of time, and then the molecule was restretched (Fig. 6). From the force-extension trace, we can determine how many domains refolded by counting the number of force peaks during the reextension. This method allows for direct determination of the refolding rate. In the example shown, note that the first unfolding event of the second stretch is an abbreviated force peak, which indicates that a partial refolding event took place when the molecule was relaxed. This is consistent with our force clamp data and previous work (21, 36) that shows that unfolding (and possibly refolding) can be a two-step process. Partial refolding events were seen infrequently but were more prevalent in the WT proteins. Combining data for all dwell times $(0-3 \mathrm{~s}), 8 \%$ of unfolded WT domains partially refolded ( 17 of 221 ), whereas only $2 \%$ of all unfolded T16I domains partially refolded ( 3 of 158) (recall that 10 and 2\% of WT and T16I, respectively, showed two-step unfolding). Refolding probability as a function of low force dwell time is shown in Table 2. To analyze data from all dwell times together, we employed MLE to determine the effective refolding rate for WT and T16I Ig10. Assuming that domains refold independently, the probability of a domain refolding under no external force is $1-e^{-\beta \cdot t}$, where $\beta$ is the refolding rate at zero force.
Using MLE with all of the refolding data results in $\beta=0.70 \pm$ $0.17 \mathrm{~s}^{-1}$ for WT Ig10 and $\beta=0.56 \pm 0.17 \mathrm{~s}^{-1}$ for T16I Ig10 ( \pm bootstrap S.D.). This suggests that the T16I mutation does not alter the refolding rate of Ig10.

Ig10 Degradation-The change in Ig10 folding kinetics is unlikely to have a relevant physiological effect in terms of titinbased passive tension. When considering the entire elastic I-band of titin's N2B isoform (the primary cardiac isoform in mice and humans $(37,38)$ ), the unfolding of a single Ig domain would change tension by $<1 \%$ between SLs of 2.0 and $2.3 \mu \mathrm{m}$. Instead, we hypothesize that unfolded Ig10 is prone to proteolysis, which would completely abolish titin's force generation mechanism and possibly lead to accelerated titin breakdown. To test if the T16I mutation affects the structural integrity of Ig10 in a way that leads to increased Ig10 proteolysis, we carried out trypsin digestion assays. Trypsin preferentially cleaves at the carboxyl side of lysine and arginine, and cleavage patterns can indicate the degree to which amino acids are structurally constrained or solvent-accessible and prone to protease cleavage.

Using a $1: 30(\mathrm{w} / \mathrm{w})$ trypsin/Ig10 5-mer ratio in digestion buffer ( $50 \mathrm{~mm}$ Tris $\cdot \mathrm{HCl}, \mathrm{pH} 7.6)$, we tested the susceptibility of WT and T16I Ig10 to proteolysis. Incubation time was varied, and all reactions were performed at $37^{\circ} \mathrm{C}$. Results are shown in Fig. 7. Although the only difference between WT and T16I Ig10 5-mers is the single point mutation in each Ig domain, the digestion assays clearly show that the mutant protein is more susceptible to trypsin cleavage, which implies that the mutation alters the Ig structure in such a way that trypsin can more easily perform peptide cleavage. To investigate whether the mutation is the direct cause of proteolysis, we performed MS on digested T16I Ig10. We excised the largest degradation product (arrowhead in Fig. 7A) from the gel, performed in-gel digestion with chymotrypsin, and identified $\mathrm{N}$-terminal peptides. This analysis identified the $\mathrm{N}$-terminal peptide to be DIPTTENLY (Fig. 7C). To be more confident that peptides at the $\mathrm{N}$-terminal side of DIPTTENLY are not present in the degradation product (e.g. a peptide may be present but not seen by MS), we used a high charge/mass MS/MS selection method on the degradation product as well as the full-length (undegraded) protein. This technique clearly identified the presence of peptides containing the His tag at the $\mathrm{N}$ terminus of the full-length protein but zero peptides for the His tag in the degradation product. This strengthens our initial findings that the DIPTTENLY peptide found is indeed the $\mathrm{N}$ terminus of the primary degradation band. Next, we performed a high resolution molecular weight measurement of the Ig10 digestion products using MALDI. We found that the molecular mass of the largest degradation product is $44,380 \mathrm{Da}$. Knowing the $\mathrm{N}$-terminal sequence of this protein band and its molecular mass, we were able to determine the C-terminal sequence (i.e. we could determine the cleavage site on the Ig10 5-mer that results in this digestion product). Starting from the $\mathrm{N}$-terminal sequence found by Orbitrap and continuing toward the $\mathrm{C}$ terminus of the T16I 5-mer, the closest molecular mass match ends with EVPEIK in the fifth serially linked Ig10 (Fig. 7C). This lysine residue (Lys-17) is therefore predicted to be the last residue in the digestion product, which means that the mutant Ig10 5-mer is cleaved one residue away 


\section{Ig10 Mutation and Domain Instability}

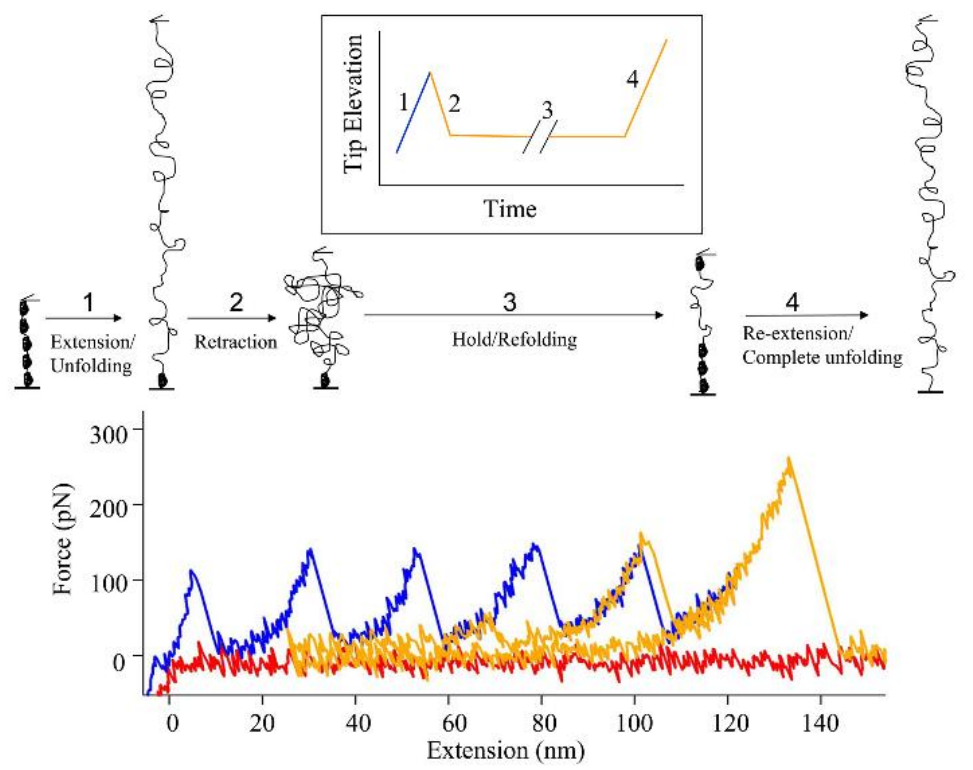

FIGURE 6. AFM refolding protocol. A tethered molecule was unfolded and then relaxed to allow for domain refolding. Restretching the molecule and identification of overlapping force peaks allows determination of how many unfolded doma ins refolded while the molecule was relaxed. The blue trace is the initial stretch. After extension and force triggers were reached, the cantilever tip was driven back toward the surface (low force portion of the orange trace, from 110 to $25 \mathrm{~nm}$ ) to release tension in the molecule. After holding for a set period of time, the molecule was restretched fully (orange trace from 25 to $140 \mathrm{~nm}$ ).

TABLE 2

Refolding percentages as a function of hold time

The number of domains that could have refolded is given in parentheses. As expected, more domains refold during longer holds. Because the data are relatively noisy, maximum likelihood estimation was used to analyze all refolding data simultaneously to extract the refolding rate at zero force. Two partial refolding event were considered equivalent to one complete refolding event. NA, not applicable.

\begin{tabular}{lll}
\hline Time & \multicolumn{1}{c}{ WT } & \multicolumn{1}{c}{ Mutant } \\
\hline \multicolumn{1}{c}{$s$} & \multicolumn{1}{c}{$\%$} & $\%$ \\
0 & $0.203(32)$ & $0.128(43)$ \\
0.25 & $0.516(32)$ & $0.538(39)$ \\
0.5 & $0.58(50)$ & NA \\
1 & $0.54(38)$ & $0.806(36)$ \\
2 & $0.807(39)$ & $0.724(29)$ \\
3 & $0.88(30)$ & $0.818(11)$ \\
\hline
\end{tabular}

from where the T16I mutation is located. This shows that the disease-linked mutation alters the structural integrity of Ig10 in such a way that susceptibility to proteolysis is increased and that proteolysis occurs where T16I disrupts the Ig10 structure. These data complement our AFM data, which show that T16I Ig10 unfolds at lower force and has a higher unfolding rate compared with wild type Ig10.

Although the trypsin degradation experiment and subsequent MS analysis allowed us to determine that T16I affects the structure of Ig10 near the mutation, we also wanted to see if T16I increased Ig10 degradation in the presence of physiologically relevant proteases. To do this, we incubated Ig7-13 (with and without T16I in Ig10) with heart extracts from mice to expose WT and T16I Ig10 to all of the proteases naturally found in the heart while being flanked by native Ig domains (Ig7-9 at the $\mathrm{N}$ terminus and Ig $11-13$ at the $\mathrm{C}$ terminus). At various time points, a portion of the $\mathrm{Ig} /$ heart extract mixture was removed and centrifuged, and the supernatant was solubilized. This pro- tocol was performed at low $\mathrm{pH}$ to mimic intracellular $\mathrm{pH}$ after ischemia ( $\mathrm{pH} \sim 6.5$ in rats (39)) and also at (relatively) high pH. The solubilized supernatants were separated by SDS-PAGE and Coomassie-stained (Fig. 8A). By measuring the optical density of the Ig7-13 band as a function of degradation time, we could determine if the T16I mutation affects the rate of titin degradation in the presence of cardiac proteases. We quantified the effect of T16I on Ig7-13 degradation by determining the half-life of full-length Ig7-13 in the heart extract mixture (Fig. $8 B)$. The half-life of WT Ig7-13 was $21.2 \mathrm{~h}$ at $\mathrm{pH} 7.38$ and $25.2 \mathrm{~h}$ at $\mathrm{pH}$ 6.33. The time it took for half of the mutant Ig7-13 to degrade was drastically less, about $6 \mathrm{~h}$ at both $\mathrm{pH}$ values. This indicates that the T16I mutation in Ig10 significantly increases the degradation rate of the Ig tandem in the presence of physiological proteases.

\section{DISCUSSION}

A recently discovered titin mutation linked with $\mathrm{AC}$ (4) is found in the tenth Ig domain of titin's proximal tandem Ig segment. We investigated how this single point mutation changes the mechanical and kinetic stability of Ig10. The AC-linked C39453T single nucleotide polymorphism in the titin gene (4) changes the native threonine (polar, hydrophilic) residue into isoleucine (nonpolar, hydrophobic). This change in hydrophobicity, in addition to the other biochemical changes associated with amino acid mutation, may play a role in disrupting Ig10 structure. The native threonine is the 16th amino acid in Ig10 and is located between the $A^{\prime}$ and B $\beta$-strands (Fig. 1) as determined by Clustal alignment with solved Ig structures (40) and secondary structure prediction of the Ig10 primary sequence (20). The residues in the A'B loop of Ig domains are important 
Ig10 Mutation and Domain Instability
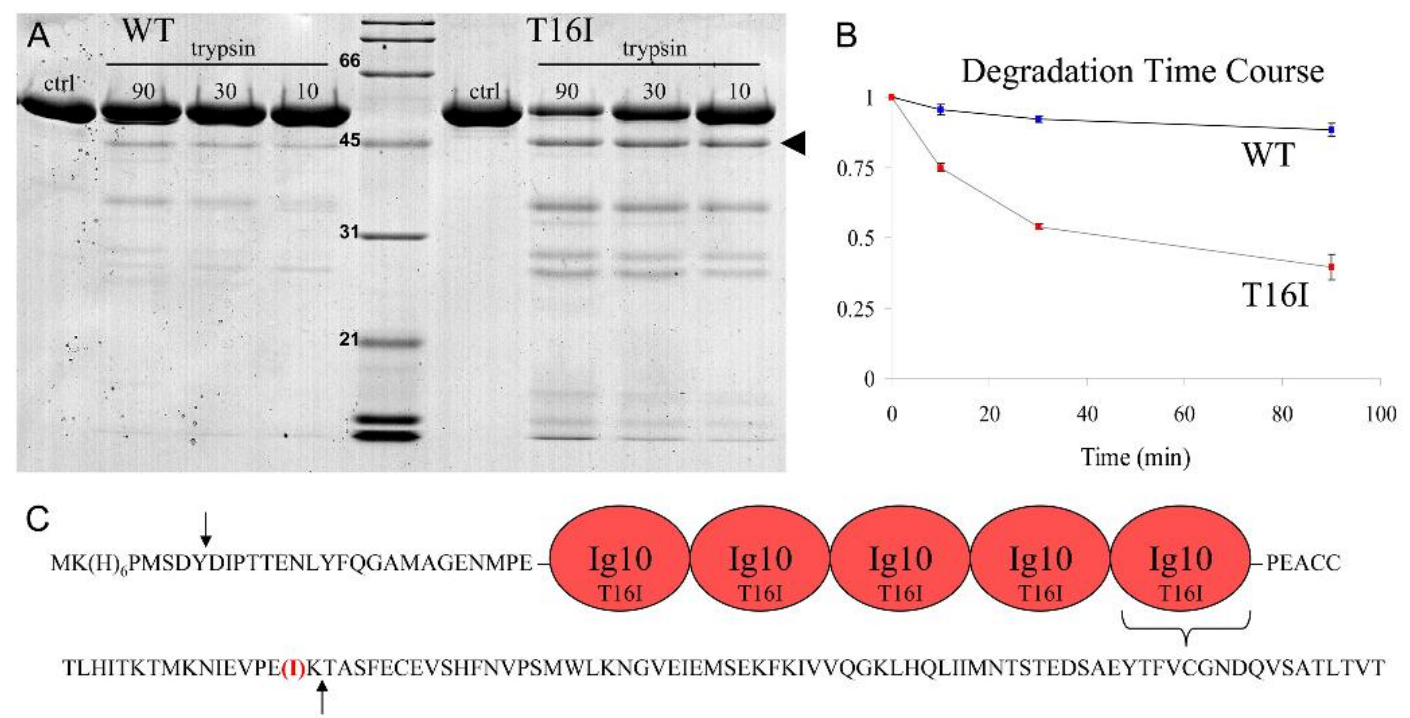

FIGURE 7. Ig10 proteolysis. A, SDS-polyacrylamide gel showing degradation products of WT and T16I Ig10 5-mer following 10-, 30-, and 90-min trypsin incubations. The arrowhead denotes the "primary degradation product" of T16l lg10 alluded to under "Discussion." WT Ig10 is relatively impervious to peptide incubations. The arrowhead denotes the "primary degradation product" of T16l lg10 alluded to under "Discussion." WT Ig10 is relatively impervious to peptide
bond cleavage. B, the a mount of full-length 5-mer remaining as a function of trypsin incubation time \pm S.E. (error bars). C, full sequence of the T16I Ig 105 -mer.

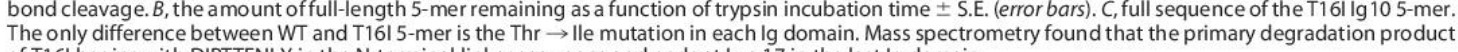
of T16l begins with DIPTTENLY in the $\mathrm{N}$-terminal linker sequence and ends at Lys-17 in the last Ig domain.

\section{A}

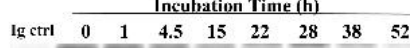

wt

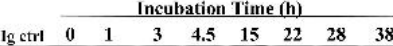

mut $\mathrm{Co}$ -

B

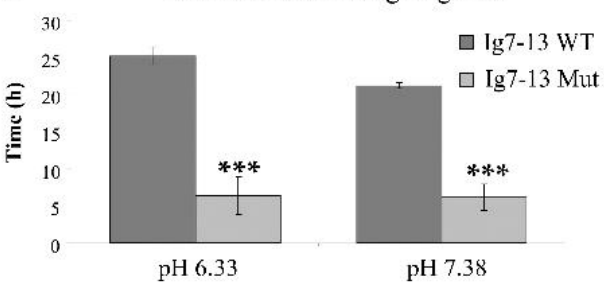

FIGURE 8. A, degradation assay with recombinant Ig7-13 (with and without $\mathrm{T} 16 \mathrm{I}$ in $\lg 10$ ) and heart extract. $\lg 7-13$ is exposed to all of the proteases naturally found in the heart. The mutant Ig7-13 protein has a larger $\mathrm{N}$-terminal linker sequence than its wild type counterpart and has mobility similar to that of a heart extract protein. These overlapping bands were fit with a double Gaussian to isolate the intensity due to full-length Ig7-13 mutant. This experiment was performed at $\mathrm{pH} \sim 6.3$ and $\sim 7.4$, but no $\mathrm{pH}$ dependence was found. $B$, Ig7-13 with the T16l lg10 domain degrades much more rapidly than WT Ig7-13 at both $\mathrm{pH}$ values, which indicates increased degradation rate $\mathrm{pH}$ values used were $6.33 \pm 0.06$ (SE) and $7.38+0.03$ (SE) $n=3$ for rach $\mathrm{pH}$. values used were $6.33 \pm 0.06$ (S.E.) and $7.38 \pm 0.03$ (S.E.); $n=3$ for each bar and Bonferroni post hoc test.

for defining and maintaining mechanical stability $(21,23,36)$, and this is supported by the fact that Thr-16 is highly conserved across species, from humans to zebrafish (4). We directly tested whether the T16I mutation alters the mechanical stability and folding kinetics of Ig10 using AFM.

FEBRUARY 22, 2013·VOLUME 288•NUMBER 8 ASBMB
Force-extension curves generated from stretching recombinant Ig10 5-mers show that the T16I mutation causes Ig10 to unfold from its native, globular state into an extended, random coil polypeptide at a lower external force. The decrease in mechanical stability of Ig10 is more relevant, considering that native Ig10 is a relatively unstable domain to begin with. Previous work has shown that other Ig domains in titin's I-band unfold at forces much higher than WT Ig10 (e.g. Ig91-98 unfolds at $\sim 225 \mathrm{pN}$ at $1000 \mathrm{~nm} / \mathrm{s}$ pulling velocity (26); Ig27, $\sim 200 \mathrm{pN}$ at $600-800 \mathrm{~nm} / \mathrm{s}(23))$ and have a much lower unfolding rate (e.g. $\alpha_{0}=6.1 \times 10^{-5} \mathrm{~s}^{-1}$ for Ig91-98, $\alpha_{0}=1.0 \times$ $10^{-5} \mathrm{~s}^{-1}$ for $\operatorname{Ig} 65-70(26) ; \alpha_{0}=2.8 \times 10^{-5} \mathrm{~s}^{-1}$ for Ig28 (41)). This means that, even prior to mutation, Ig10 is more likely to occupy a non-native, extended conformation. The mutation further increases the instability of Ig10 relative to other domains, which supports the hypothesis that the mutation may be physiologically relevant. For example, if the mutation simply lowered the propensity of Ig10 for unfolding, but T16I Ig10 behaved similarly to other Ig domains, then the mutation would probably not have a significant deleterious effect.

The force clamp data showed that two-step unfolding is five times more frequent in WT Ig10, which was somewhat surprising and deserving of comment. It has been shown that the $\mathrm{A}^{\prime}$ $\beta$-strand residues of Ig domains strongly influence their ability to withstand mechanical stress via hydrogen bond networks with the $\mathrm{G} \beta$-strand $(21,23)$, and the location of the T16I mutation suggests disruption of this important native contact network. These terminal $\beta$-strands may also locally unfold and extend prior to complete molecular denaturing (36), resulting in short extension increases before the hydrophobic core falls apart. Our data suggest that T16I weakens the firm A'G con- 


\section{Ig10 Mutation and Domain Instability}

tacts, such that $\beta$-strands sliding past each other may not always be a distinct structural transition. Instead, external force does not build up in the A'G strand (e.g. strand stiffening (42)) but instead is transmitted more directly to the stable protein core. Subsequently, a force peak does not develop when the A'G $\beta$-strands separate, and only a one-step unfolding event is recorded

Although arrhythmogenic cardiomyopathy is associated with a broad phenotypic spectrum (for a recent review, see Ref. 43), the most prominent pathological change is myocyte loss, which is often accompanied by fibrofatty replacement or inflammation. The disease progression is poorly understood, although it is clear that myocyte degradation is a hallmark feature (44). Our hypothesis is that the AC-linked mutation decreases the force needed to unfold Ig10 (or, similarly, increases the Ig10 unfolding rate) and increases proteolytic susceptibility. Protease cleavage of sarcomeric proteins has been linked to cardiac pathology previously, including myosin light chain (45) and myosin-binding protein-C (46). Peptide bond breakage anywhere within titin's elastic I-band would abolish titin's force generation mechanism and would probably lead to severe cardiac dysfunction. It is unclear how the cell responds to I-band cleavage, but accelerated titin turnover and/or apoptosis are possible. This myocyte loss may be the first step of AC development therefore, we wanted to test if the T16I mutation changes the degradation proclivity of Ig10.

The trypsin digestion assays show that mutant Ig10 is more prone to proteolysis, and mass spectrometry confirmed that the cleavage site is in the $\mathrm{A}^{\prime} \mathrm{B}$ loop where the mutation is located. This result is noteworthy because it shows that T16I Ig10 is more prone to degradation even under zero external force. Regardless, proteolysis would occur more readily if the protein exists in a fully unfolded state. To this point, it is worthwhile to determine to what degree the T16I mutation increases the amount of time that Ig10 is globally unfolded. From our force clamp and refolding AFM data, we determined the global unfolding and refolding rate of WT Ig10 to be $\alpha_{0}=0.004 \mathrm{~s}^{-1}$ and $\beta_{0}=0.70 \mathrm{~s}^{-1}$, respectively. These parameters for T16I Ig10 are $\alpha_{0}=0.018 \mathrm{~s}^{-1}$ and $\beta_{\mathrm{o}}=0.56$ $\mathrm{s}^{-1}$. In order to estimate the fraction of Ig10 domains unfolded in vivo, we performed computer simulations (see "Experimental Procedures"). Our simulations predict that $1.8 \%$ of WT Ig10 domains and $9.5 \%$ of T16I Ig10 domains are unfolded at any time (i.e. the T16I mutation increases the probability that Ig10 is unfolded in a beating ventricle by a factor of 5). In addition, identical analysis using the unfolding and refolding rates of other titin Ig domains $(23,26)$ predicts that $\leq 0.1 \%$ of $\operatorname{Ig} 27$, Ig91-98, or Ig65-70 is unfolded at any time, a 100-fold decrease compared with T16I Ig10 (Fig. 9).

We also determined the free energy of unfolding for WT and T16I Ig10. To do this, we generated recombinant WT and T16 Ig10 monomers and measured the amount of protein that is unfolded under no force using pulse proteolysis (47). Briefly, Ig10 monomers were prepared in varying concentrations of denaturant (urea) and allowed to reach conformational equilibrium. Then excess protease (thermolysin) was added to digest

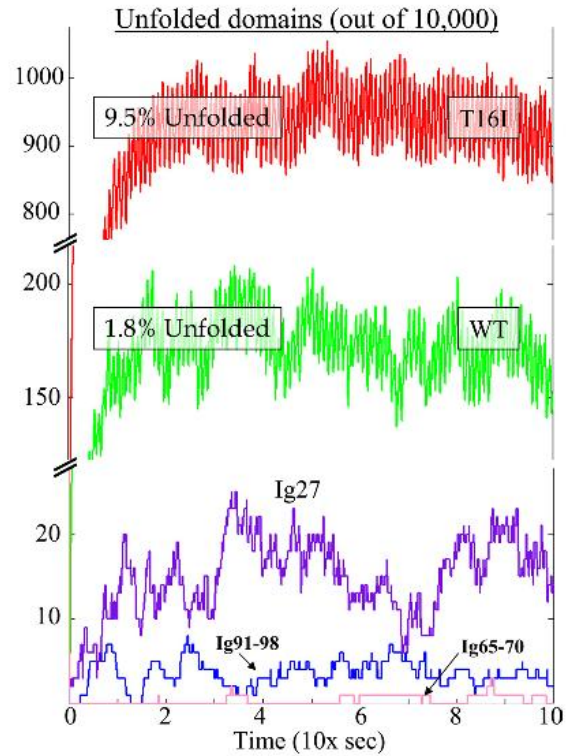

FIGURE 9. In vivo unfolding simulations. The force experienced by titin's elastic I-band region in the beating heart was simulated and used to estimate the percentage of Ig domains that are unfolded in vivo. WT Ig10 is unfolded an order of magnitude more often than other Ig domains, which shows that $\lg 10$ is less stable than other Ig domains even without the T 16 I mutation. T16I Ig10 is unfolded 2 orders of magnitude more than other Ig domains. Note the breaks in the $y$ axis.

the fraction of Ig10 monomers that were unfolded at the given urea concentration. This method quantifies the percentage of WT and T16I Ig10 monomers that are unfolded under different urea concentrations, and it also allows estimation of the Gibbs free energy of unfolding. Fig. $10 \mathrm{~A}$ shows the fraction of WT Ig10 monomers that remain folded as a function of urea concentration as determined by pulse proteolysis. The fraction of folded protein at urea concentrations in the transition zone (where folded and unfolded populations are both substantial) was extrapolated to determine the folded fraction at $0 \mathrm{M}$ urea (Fig. 10B), which gives the free energy of unfolding in water and is related to the relative population of unfolded and folded WT Ig10 using Equation 4,

$$
\Delta G^{0}=-k_{B} T \ln \frac{[U]}{[F]}
$$

where $[U]$ and $[F]$ represent the concentration of unfolded and folded protein, respectively. We estimated that the free energy of unfolding for WT Ig10 is $2.59 \pm 0.4 \mathrm{kcal} / \mathrm{mol}$, which corresponds to $\sim 1 \%$ of WT monomers being unfolded at any given time at room temperature (consistent with our simulations (Fig. 9)). This exact method did not work for the T16I Ig10 monomer, however, because the mutant monomer was strongly degraded by thermolysin even in the absence of any urea. Nonetheless, we were able to determine the amount of T16I Ig10 that was degraded at $0 \mathrm{M}$ urea and estimated the free energy of unfolding for T16I Ig10 to be $0.156 \pm 0.2 \mathrm{kcal} / \mathrm{mol}$, which corresponds to $40-50 \%$ of T16I monomers being 


\section{Ig10 Mutation and Domain Instability}
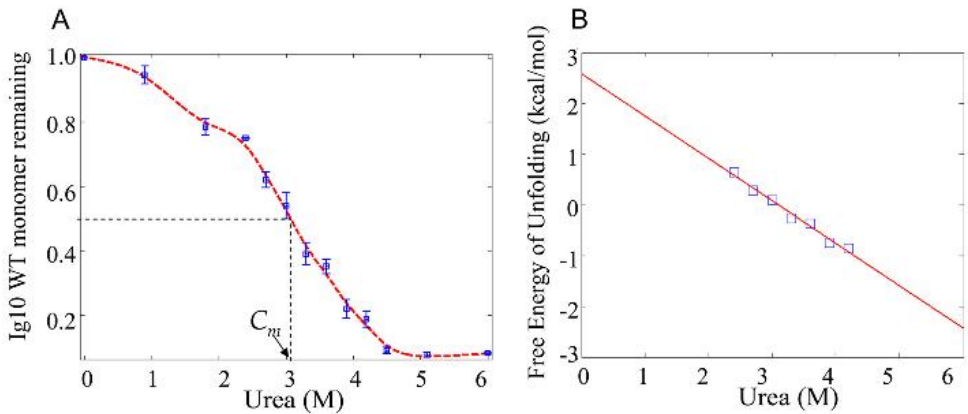

FIGURE 10. Pulse proteolysis. $A$, the fraction of WT Ig 10 monomer that remains folded decreases as urea concentration increases. The $C_{m}$ value indicates the urea concentration where folded and unfolded protein levels are equal. $B$, the free energy of unfolding at various urea concentrations in the transition zone (where unfolded and folded populations are both substantial) is determined from the percentage of folded and unfolded lg 10 ; extrapolating to $0 \mathrm{~m}$ urea allows determination of the free energy of unfolding in the absence of any denaturant. Errorbars, S.E.

unfolded at any given time. This result is consistent with previous NMR work that showed that T16I Ig10 co-exists as both a well structured and less structured protein in solution (4). The fraction of unfolded T16I monomers estimated by pulse proteolysis is more than that in our in vivo simulations, and this is probably because our simulations predict global unfolding whereas the pulse proteolysis protocol does not discriminate between local and global unfolding (i.e. a flexible peptide loop may be cleaved even if the protein core is still intact). Nonetheless, these data once again show that the AC-linked point mutation greatly affects the conformational stability of Ig10 and increases its proteolytic susceptibility. We repeated this pulse proteolysis protocol with our Ig7-13 constructs and found that the T16I mutation significantly decreased the ability of Ig7-13 to remain folded under chemical denaturation. The urea concentration at which half of Ig7-13 was degraded was $4.89 \pm$ $0.08 \mathrm{~m}$ (S.E.) for WT Ig7-13 and $4.54 \pm 0.06 \mathrm{M}$ (S.E.) for mutant $\operatorname{Ig} 7-13(p=0.008$; data not shown). This suggests that Ig10 unfolds more easily due to the T16I mutation even when interdomain Ig contacts are conserved.

The trypsin digestion assays show that T16I Ig10 is more prone to degradation, but it was important to find if Ig10 structure is compromised at the location of the T16I mutation. From MS analysis, we found the $\mathrm{N}$-terminal sequence of the primary Ig10 5-mer degradation product (arrowhead in Fig. $7 A$ ) to be in the N-terminal linker sequence that was added to the Ig10 5-mer after synthetic Ig10 DNA was inserted into the pETM11 vector (which also added a His tag for protein purification). We also used MALDI to determine the molecular mass of all Ig10 digestion products and found that the primary degradation product is $44,380 \mathrm{Da}$. Starting from the $\mathrm{N}$-terminal sequence found by MS, the molecular mass measurement matches the T16I 5-mer primary sequence at the lysine residue immediately following the T16I residue in the fifth Ig10 domain. The peptide D ... K (demarcated by arrows in Fig. 7C) that results from tryptic cleavage has a predicted mass of $44,307 \mathrm{Da}$. This predicted mass is slightly less than the measured value, but MS showed that various residues within Ig10 can be modified (mostly oxidation, +16 Da). Using MALDI, we found the molecular masses of the full-length 5-mer and three of the four most prominent degradation bands to be $54,171,44,380$,
34,425 , and 24,087 $\mathrm{Da}$. The molecular mass difference between these bands is 9791,9955 , and $10,338 \mathrm{Da}$, respectively. The predicted molecular mass of the peptide sequence between the Lys-17 (the C-terminal cleavage site in the primary digestion band) residues of adjacent serially linked Ig10 is 10,018 Da, which suggests that each of these protein bands is formed from cleavage of the full T16I5-mer at the location of the mutation in one of the tandem Ig10 domains, resulting in one Ig10 molecular mass difference between bands. One of the four most prominent degradation bands has a molecular mass of 27,105 $\mathrm{Da}$, which is not $\sim 10 \mathrm{kDa}$ different from the other degradation products. This band may be due to additional protein degradation following cleavage at Lys-17 because any peptide bond break probably compromises the entire $\beta$-barrel structure. The observed digestion band pattern is much more pronounced in the mutant Ig10 5-mer (Fig. 7A), which shows that increased proteolysis results from the AC-linked mutation, and MS showed that the cleavage site is within the amino acid loop that harbors T16I.

To provide evidence for physiologically relevant degradation, we studied if the disease-linked mutation affects Ig10 degradation when Ig10 is flanked by native Ig domains and when physiological proteases are present. We incubated our Ig7-13 construct with blended mouse heart to expose $\operatorname{Ig} 7-13$ to all of the proteases in the heart. Ig7-13 was degraded much more rapidly in the mutant construct (Fig. $8 B$ ), presumably due to the T16I mutation in Ig10. Similar to our pulse proteolysis data, this result shows that T16I increases titin degradation even in the absence of force. More work is needed to determine the proteolytic pathway(s) responsible for this degradation.

With the recent discovery of the T16I Ig10 mutation found in patients with arrhythmogenic cardiomyopathy overlap syndromes, we set out to determine the effects that the mutation had on the single molecule dynamics of Ig10. We found that the mutation lowered the force needed to unfold Ig10, increased its unfolding rate, and increased its susceptibility to proteolysis. These single molecule studies were a necessary first step in the ongoing investigation of the connection between titin and AC. Future studies will focus on the effect of the T16I mutation on titin breakdown in the myocardium and pathological changes 


\section{Ig10 Mutation and Domain Instability}

of diseased hearts. Elucidating the mechanistic pathway that connects a single mutation in Ig10 to cardiac remodeling, ventricular arrhythmias, and sudden cardiac death is crucial for improving diagnosis, developing treatments, and preventing fatality.

\section{REFERENCES}

1. Krüger, M., and Linke, W. A. (2009) Titin-based mechanical signalling in normal and failing myocardium. J. Mol. Cell. Cardiol. 46, 490-498

2. Granzier, H. L., and Labeit, S. (2004) The giant protein titin. A major player in myocardial mechanics, signaling, and disease. Circ. Res. 94, 284-295

3. Herman, D. S., Lam, L., Taylor, M. R., Wang, L., Teekakirikul, P., Christodoulou, D., Conner, L., DePalma, S. R., McDonough, B., Sparks, E., Teodorescu, D. L., Cirino, A. L., Banner, N. R., Pennell, D. J., Graw, S., Merlo, M., Di Lenarda, A., Sinagra, G., Bos, J. M., Ackerman, M. J., Mitchell, R. N., Murry, C. E., Lakdawala, N. K., Ho, C. Y., Barton, P. J., Cook, S. A., Mestroni, L., Seidman, J. G., and Seidman, C. E. (2012) Truncations of titin causing dilated cardiomyopathy. N. Engl. J. Med. 366, 619-628

4. Taylor, M., Graw, S., Sinagra, G., Barnes, C., Slavov, D., Brun, F., Pinamonti, B., Salcedo, E. E., Sauer, W., Pyxaras, S., Anderson, B., Simon, B. Bogomolovas, J., Labeit, S., Granzier, H., and Mestroni, L. (2011) Genetic variation in titin in arrhythmogenic right ventricular cardiomyopathyoverlap syndromes. Circulation 124, 876-885

5. McKoy, G., Protonotarios, N., Crosby, A., Tsatsopoulou, A., Anastasakis, A., Coonar, A., Norman, M., Baboonian, C., Jeffery, S., and McKenna, W.J. (2000) Identification of a deletion in plakoglobin in arrhythmogenic right ventricular cardiomyopathy with palmoplantar keratoderma and woolly hair (Naxos disease). Lancet 355, 2119-2124

6. Norgett, E. E., Hatsell, S. J., Carvajal-Huerta, L., Cabezas, J. C., Common, J. Purkis, P. E., Whittock, N., Leigh, I. M., Stevens, H. P., and Kelsell, D. P. (2000) Recessive mutation in desmoplakin disrupts desmoplakin-intermediate filament interactions and causes dilated cardiomyopathy, woolly hair and keratoderma. Hum. Mol. Genet. 9, 2761-2766

7. Gerull, B., Heuser, A., Wichter, T., Paul, M., Basson, C. T., McDermott, D. A., Lerman, B. B., Markowitz, S. M., Ellinor, P. T., MacRae, C. A., Peters, S., Grossmann, K. S., Drenckhahn, J., Michely, B., Sasse-Klaassen, S. Birchmeier, W., Dietz, R., Breithardt, G., Schulze-Bahr, E., and Thierfelder, L. (2004) Mutations in the desmosomal protein plakophilin-2 are common in arrhythmogenic right ventricular cardiomyopathy. Nat Genet. 36, 1162-1164.

8. Pilichou, K., Nava, A., Basso, C., Beffagna, G., Bauce, B., Lorenzon, A. Frigo, G., Vettori, A., Valente, M., Towbin, J., Thiene, G., Danieli, G. A., and Rampazzo, A. (2006) Mutations in desmoglein-2 gene are associated with arrhythmogenic right ventricular cardiomyopathy. Circulation 113, 1171-1179

9. Heuser, A., Plovie, E. R., Ellinor, P. T., Grossmann, K. S., Shin, J. T., Wichter, T., Basson, C. T., Lerman, B. B., Sasse-Klaassen, S., Thierfelder, L., MacRae, C. A., and Gerull, B. (2006) Mutant desmocollin-2 causes arrhythmogenic right ventricular cardiomyopathy. Am. J. Hum. Genet. 79, $1081-1088$

10. Basso, C., Corrado, D., and Thiene, G. (1999) Cardiovascular causes of sudden death in young individuals including athletes. Cardiol. Rev. 7 , $127-135$

11. Nagueh, S. F., Shah, G., Wu, Y., Torre-Amione, G., King, N. M., Lahmers, S., Witt, C. C., Becker, K., Labeit, S., and Granzier, H. L. (2004) Altered titin expression, myocardial stiffness, and left ventricular function in patients with dilated cardiomyopathy. Circulation 110, 155-162

12. Krüger, M., Kötter, S., Grützner, A., Lang, P., Andresen, C., Redfield M. M., Butt, E., dos Remedios, C. G., and Linke, W. A. (2009) Protein kinase $\mathrm{G}$ modulates human myocardial passive stiffness by phosphorylation of the titin springs. Circ. Res. 104, 87-94

13. Hidalgo, C., Hudson, B., Bogomolovas, J., Zhu, Y., Anderson, B., Greaser, M., Labeit, S., and Granzier, H. (2009) PKC phosphorylation of titin's PEVK element. A novel and conserved pathway for modulating myocardial stiffness. Circ. Res. 105, 631-638, 17 p following 638

14. Labeit, S., and Kolmerer, B. (1995) Titins. Giant proteins in charge of muscle ultrastructure and elasticity. Science 270, 293-296

15. Improta, S., Politou, A. S., and Pastore, A. (1996) Immunoglobulin-like modules from titin I-band. Extensible components of muscle elasticity. Structure 4, 323-337

16. Marino, M., Svergun, D. I., Kreplak, L., Konarev, P. V., Maco, B., Labeit, D., and Mayans, O. (2005) Poly-Ig tandems from I-band titin share extended domain arrangements irrespective of the distinct features of their modular constituents. J. Muscle Res. Cell Motil. 26, 355-365

17. von Castelmur, E., Marino, M., Svergun, D. I., Kreplak, L., Ucurum-Fotiadis, Z., Konarev, P. V., Urzhumtsev, A., Labeit, D., Labeit, S., and Mayans, O. (2008) A regular pattern of Ig super-motifs defines segmental flexibility as the elastic mechanism of the titin chain. Proc. Natl. Acad. Sci. U.S.A. 105, 1186-1191

18. Trombitás, K., Greaser, M., Labeit, S., Jin, J. P., Kellermayer, M., Helmes, M., and Granzier, H. (1998) Titin extensibility in situ. Entropic elasticity of permanently folded and permanently unfolded molecular segments. J. Cell Biol. 140, 853-859

19. Pieper, U., Webb, B. M., Barkan, D. T., Schneidman-Duhovny, D., Schlessinger, A., Braberg, H., Yang, Z., Meng, E. C., Pettersen, E. F., Huang, C. C., Datta, R. S., Sampathkumar, P., Madhusudhan, M. S., Sjölander, K., Ferrin, T. E., Burley, S. K., and Sali, A. (2011) ModBase, a database of annotated comparative protein structure models, and associated resources. Nucleic Acids Res. 39, D465-D474

20. Cole, C., Barber, J. D., and Barton, G. J. (2008) The Jpred 3 secondary structure prediction server. Nucleic Acids Res. 36, W197-W201

21. Lu, H., Isralewitz, B., Krammer, A., Vogel, V., and Schulten, K. (1998) Unfolding of titin immunoglobulin domains by steered molecular dynamics simulation. Biophys. J. 75, 662-671

22. Fowler, S. B., Best, R. B., Toca Herrera, J. L., Rutherford, T. J, Steward, A., Paci, E., Karplus, M., and Clarke, J. (2002) Mechanical unfolding of a titin Ig domain. Structure of unfolding intermediate revealed by combining AFM, molecular dynamics simulations, NMR, and protein engineering. J. Mol. Biol. 322, 841-849

23. Li, H., Carrion-Vazquez, M., Oberhauser, A. F., Marszalek, P. E., and Fernandez, J. M. (2000) Point mutations alter the mechanical stability of immunoglobulin modules. Nat. Struct. Biol. 7, 1117-1120

24. Carrion-Vazquez, M., Oberhauser, A. F., Fowler, S. B., Marszalek, P. E., Broedel, S. E., Clarke, J., and Fernandez, J. M. (1999) Mechanical and chemical unfolding of a single protein. A comparison. Proc. Natl. Acad. Sci. U.S.A. 96, 3694-3699

25. Grützner, A., Garcia-Manyes, S., Kötter, S., Badilla, C. L., Fernandez, J. M., and Linke, W. A. (2009) Modulation of titin-based stiffness by disulfide bonding in the cardiac titin N2-B unique sequence. Biophys. J. 97, $825-834$

26. Watanabe, K., Muhle-Goll, C., Kellermayer,M. S., Labeit, S., and Granzier, H. (2002) Different molecular mechanics displayed by titin's constitutively and differentially expressed tandem Ig segments. J. Struct. Biol. 137, $248-258$

27. LeWinter, M.M., and Granzier, H. (2010) Cardiac titin. A multifunctional giant. Circulation 121, 2137-2145

28. Zhu, Y., Bogomolovas, J., Labeit, S., and Granzier, H. (2009) Single molecule force spectroscopy of the cardiac titin N2B element. Effects of the molecular chaperone alphaB-crystallin with disease-causing mutations. J. Biol. Chem. 284, 13914-13923

29. Dubois, L., and Nuzzo, R. (1992) Synthesis, structure, and properties of model organic surfaces. Annu. Rev. Phys. Chem. 43, 437-463

30. Zhmurov, A., Dima, R. I., and Barsegov, V. (2010) Order statistics theory of unfolding of multimeric proteins. Biophys. J. 99, 1959-1968

31. Marko, J. F., and Siggia, E. D. (1995) Stretching DNA. Macromolecules 28, $8759-8770$

32. Nedrud, J., Labeit, S., Gotthardt, M., and Granzier, H. (2011) Mechanics on myocardium deficient in the $\mathrm{N} 2 \mathrm{~B}$ region of titin. The cardiac-unique spring element improves efficiency of the cardiac cycle. Biophys. J. 101, $1385-1392$

33. Irving, T., Wu, Y., Bekyarova, T., Farman, G. P., Fukuda, N., and Granzier, H. (2011) Thick-filament strain and interfilament spacing in passive muscle. Effect of titin-based passive tension. Biophys. J. 100, 1499-1508

34. Chung, C. S., and Granzier, H. L. (2011) Contribution of titin and extra- 


\section{Ig10 Mutation and Domain Instability}

cellular matrix to passive pressure and measurement of sarcomere length in the mouse left ventricle. J. Mol. Cell Cardiol. 50, 731-739

35. Massey, F. J. (1951) The Kolmogorov-Smirnov test for goodness of fit. J. Am. Stat. Assoc. 46, 68-78

36. Marszalek, P. E., Lu, H., Li, H., Carrion-Vazquez, M., Oberhauser, A. F. Schulten, K., and Fernandez, J. M. (1999) Mechanical unfolding intermediates in titin modules. Nature 402, 100-103

37. Cazorla, O., Freiburg, A., Helmes, M., Centner, T., McNabb, M., Wu, Y. Trombitás, K., Labeit, S., and Granzier, H. (2000) Differential expression of cardiac titin isoforms and modulation of cellular stiffness. Circ. Res. 86, $59-67$

38. Chung, C. S., Bogomolovas, J., Gasch, A., Hidalgo, C. G., Labeit, S., and Granzier, H. L. (2011) Titin-actin interaction. PEVK-actin-based viscosity in a large animal. J. Biomed. Biotechnol. 2011, 310791

39. Schroeder, M. A., Swietach, P., Atherton, H. J., Gallagher, F. A., Lee, P., Radda, G. K., Clarke, K., and Tyler, D. J. (2010) Measuring intracellular pH in the heart using hyperpolarized carbon dioxide and bicarbonate: a C-13 and P-31 magnetic resonance spectroscopy study. Cardiovasc. Res. 86, $82-91$

40. Chenna, R., Sugawara, H., Koike, T., Lopez, R., Gibson, T. J., Higgins, D. G and Thompson, J. D. (2003) Multiple sequence alignment with the Clustal series of programs. Nucleic Acids Res. 31, 3497-3500

41. Li, H., Oberhauser, A. F., Fowler, S. B., Clarke, J., and Fernandez, J. M.
(2000) Atomic force microscopy reveals the mechanical design of a modular protein. Proc. Natl. Acad. Sci. U.S.A. 97, 6527-6531

42. Stacklies, W., Vega, M. C., Wilmanns, M., and Gräter, F. (2009) Mechanical network in titin immunoglobulin from force distribution analysis. PLoS Comput. Biol. 5, e1000306

43. Sen-Chowdhry, S., Morgan, R. D., Chambers, J. C., and McKenna, W. J. (2010) Arrhythmogenic cardiomyopathy. Etiology, diagnosis, and treatment. Annu. Rev. Med. 61, 233-253

44. Herren, T., Gerber, P. A., and Duru, F. (2009) Arrhythmogenic right ventricular cardiomyopathy/dysplasia. A not so rare "disease of the desmosome" with multiple clinical presentations. Clin. Res. Cardiol. 98, $141-158$

45. Margossian, S. S., White, H. D., Caulfield, J. B., Norton, P., Taylor, S., and Slayter, H. S. (1992) Light chain 2 profile and activity of human ventricular myosin during dilated cardiomyopathy. Identification of a causal agent for impaired myocardial function. Circulation 85, 1720-1733

46. Govindan, S., Sarkey, J., Ji, X., Sundaresan, N. R., Gupta, M. P., de Tombe, P. P., and Sadayappan, S. (2012) Pathogenic properties of the N-terminal region of cardiac myosin binding protein-C in vitro. J. Muscle Res. Cell Motil. 33, 17-30

47. Park, C., and Marqusee, S. (2006) Quantitative determination of protein stability and ligand binding by pulse proteolysis. Curr. Protoc. Protein Sci. Chapter 20, Unit 20.11 


\section{Appendix B}

Brian R. Anderson and Henk L. Granzier, Titin-based tension in the cardiac sarcomere: molecular origin and physiological adaptations. Prog Biophys Mol Biol, 2012. 110(2-3): p. 204-17. 
Progress in Biophysics and Molecular Biology 110 (2012) 204-217

Review

\title{
Titin-based tension in the cardiac sarcomere: Molecular origin and physiological adaptations
}

\author{
Brian R. Anderson ${ }^{a, b}$, Henk L. Granzier ${ }^{\text {b,* }}$ \\ ${ }^{a}$ Department of Physics, University of Arizona, Tucson, AZ 85724, USA \\ b Molecular Cardiovascular Research Program, Sarver Heart Center, Department of Physiology, University of Arizona, Tucson, AZ 85724, USA
}

\section{A R T I C L E I N F O}

\section{Article history:}

Available online 11 August 2012

\section{Keywords:}

Passive tension

Connectin

Entropic force

Mechanical signaling

\section{A B S T R A C T}

The passive stiffness of cardiac muscle plays a critical role in ventricular filling during diastole and is determined by the extracellular matrix and the sarcomeric protein titin. Titin spans from the Z-disk to the M-band of the sarcomere and also contains a large extensible region that acts as a molecular spring and develops passive force during sarcomere stretch. This extensible segment is titin's I-band region, and its force-generating mechanical properties determine titin-based passive tension. The properties of titin's I-band region can be modulated by isoform splicing and post-translational modification and are intimately linked to diastolic function. This review discusses the physical origin of titin-based passive tension, the mechanisms that alter titin stiffness, and titin's role in stress-sensing signaling pathways. (c) 2012 Elsevier Ltd. All rights reserved.

\section{Contents}

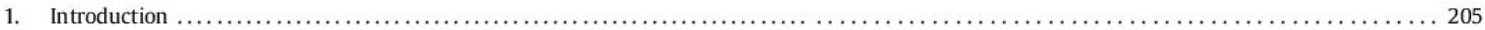

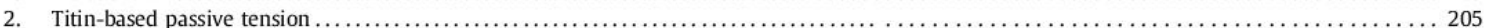

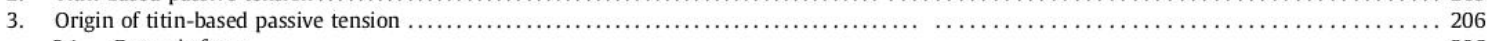

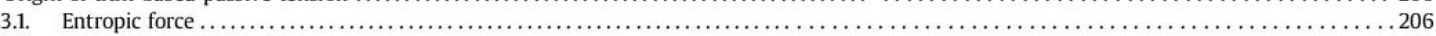

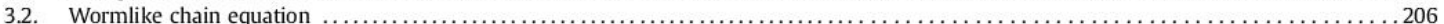

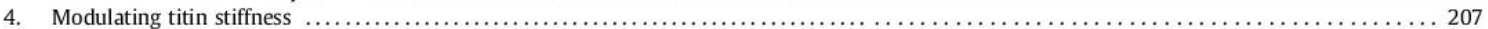

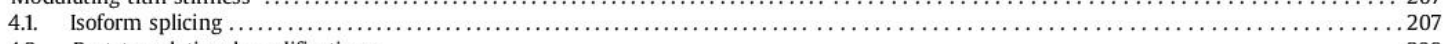

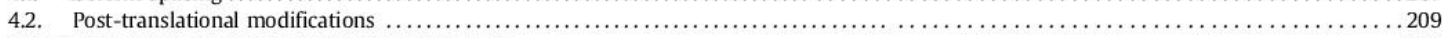

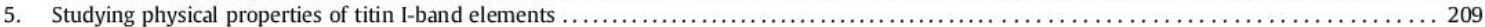

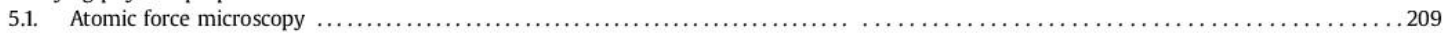

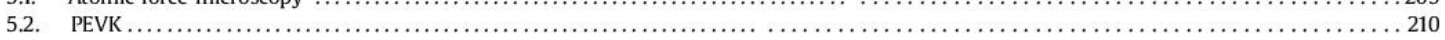

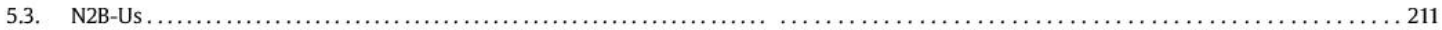

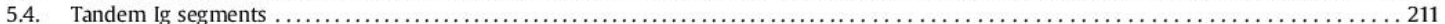

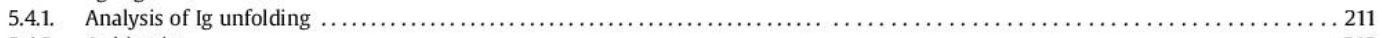

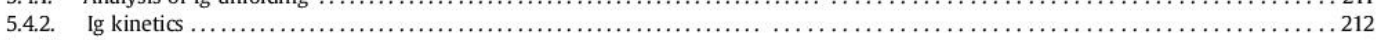

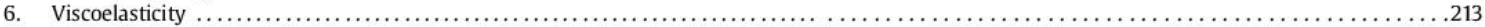

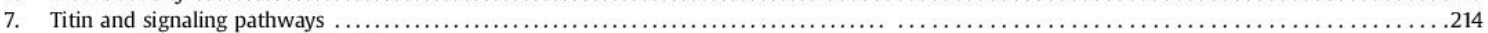

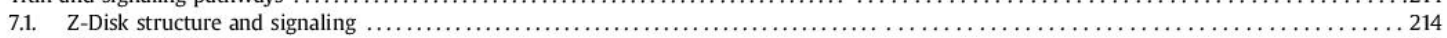

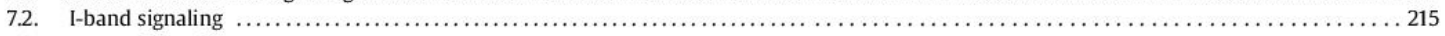

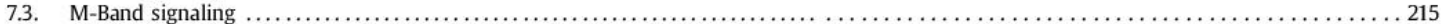

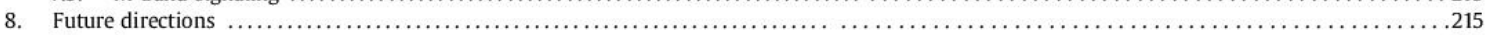

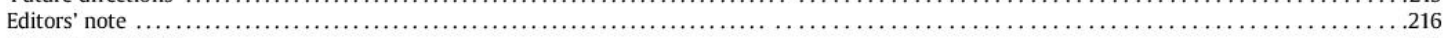

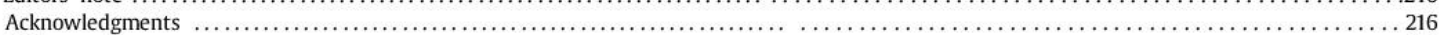

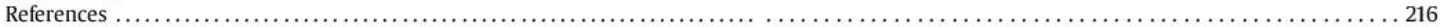

\footnotetext{
* Corresponding author. Tel.: +1520626 3641; fax: +1520626 7600.

E-mail address: granzier@email.arizona.edu (H.L. Granzier).
}

0079-6107/\$ - see front matter @ 2012 Elsevier Ltd. All rights reserved. http://dx.doi.org/10.1016/j.pbiomolbio.2012.08.003 


\section{Introduction}

The cardiac sarcomere contains, in addition to actin-based thin filaments and myosin-based thick filaments, the giant protein titin (also called connectin). While the proteins that compose the thin and thick filaments are responsible for generating active force in the sarcomere, titin provides passive elasticity to muscle. This elastic property of titin, in addition to the extracellular matrix, defines the passive stiffness of muscle that resists sarcomere stretch. Most of the extracellular matrix's contribution to passive tension is due to collagen, with collagen playing a larger role towards the upper limit of the physiological sarcomere length range (Wu et al., 2000, 2002). While the extracellular matrix is also important to passive stiffness, this review will focus on titin. In addition to providing elasticity to muscle, titin is also essential for maintaining the structural integrity of the sarcomere. Diastolic filling of the beating heart is largely determined by titin-based passive tension, with changes in titin stiffness associated with diastolic dysfunction and cardiac disease.

A single titin molecule spans the half sarcomere, binding to the $\mathrm{Z}$-disk and to the thin filament at its $\mathrm{N}$-terminus, and binding to the thick filament and M-band towards its C-terminus (Fig. 1A). Between its thin and thick filament binding domains titin contains a large segment that behaves as a molecular spring that extends during sarcomere stretch. This spring-like segment is titin's I-band region; it centers the thick filament in the middle of the sarcomere and generates a passive restoring force that resists increases in sarcomere length. However, this portion of titin is not only a molecular spring but can also interact with thin filament proteins or possibly adjacent titin molecules. Nonetheless, it primarily defines titin-based passive tension. Titin's I-band region contains three extensible elements: 1 ) serially-linked immunoglobulin(Ig)like domains, 2) the PEVK element (rich in proline (P), glutamate (E), valine (V), and lysine (K) residues), and 3 ) the N2B element. Changes in titin's I-band region, namely isoform splicing and posttranslational modifications, directly influence titin-based passive tension. Although the I-band region of titin generates most titinbased passive tension, other regions of titin (Z-disk, A-band, and M-band) are involved in numerous cellular processes including maintaining sarcomere structure and force-dependent signaling. This review focuses on the physical concepts at the core of titin's elasticity, the physiological mechanisms that tune titin-based tension, and titin's role in mechanical stress sensing.

\section{Titin-based passive tension}

The importance of titin-based passive tension cannot be overstated. Drastic changes in titin isoform expression occur during neonatal cardiac development as large, compliant titin isoforms are replaced by smaller, stiffer isoforms to meet the increased needs of the newborn heart (Lahmers et al., 2004; Opitz et al., 2004; Greaser et al., 2005). Certain cardiac diseases exhibit

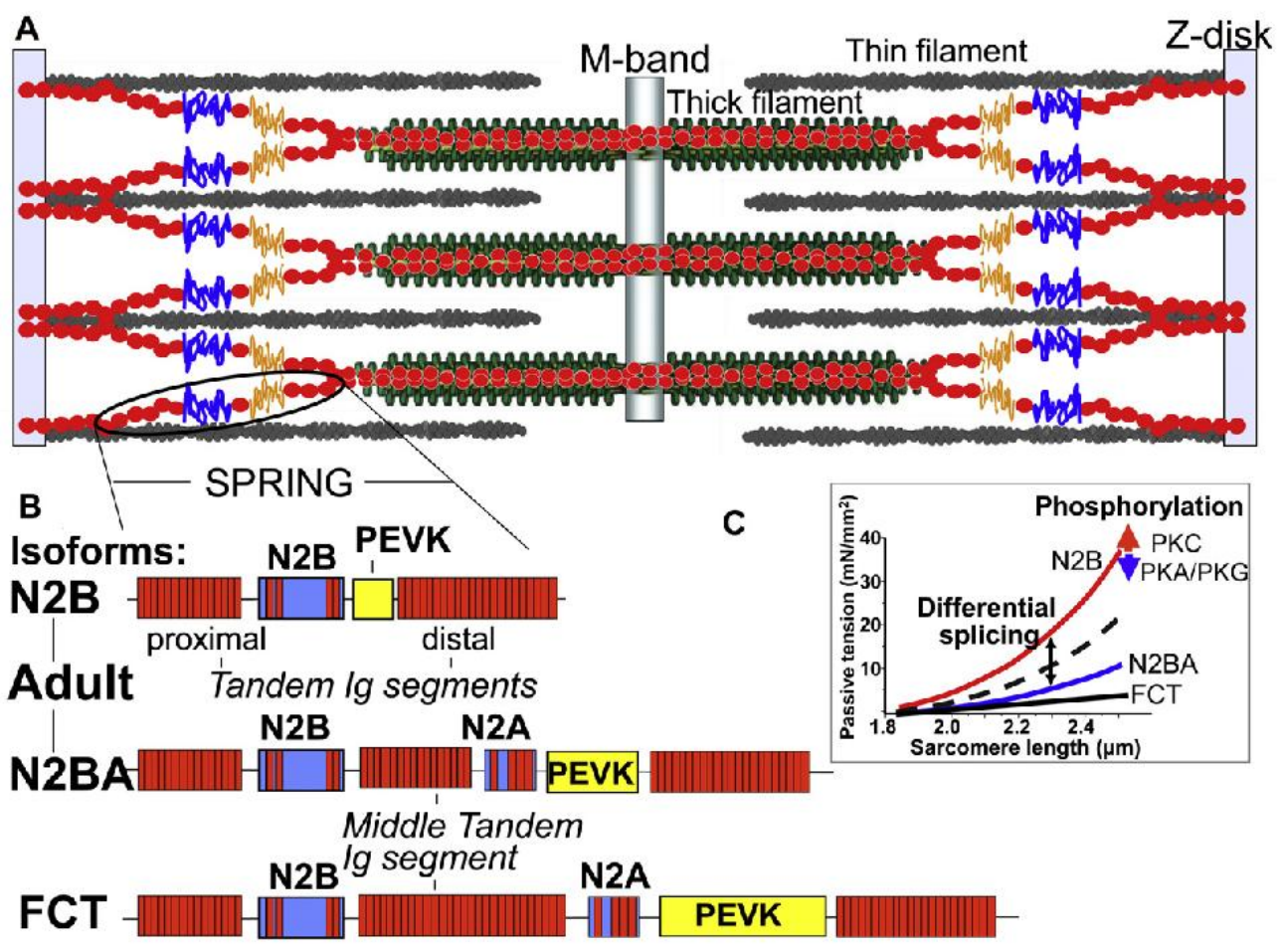

Fig. 1. A) A schematic of the sarcomere. A single titin molecule spans from the Z-disk to the M-band and contains a spring-like region that develops force upon sarcomere stretch. B) The I-band region composition of the three cardiac titin isoforms. The adult N2BA isoform and fetal cardiac titin isoform contain a middle tandem Ig segment, the N2A element, and a longer PEVK sequence than the short N2B isoform. C) Titin-based passive tension levels are determined by titin isoform composition and can also be modulated by phosphorylation of I-band elements. 
changes in titin isoform expression that can be compensatory or contribute to the disease phenotype (Neagoe et al., 2002; Warren et al., 2003; Nagueh et al., 2004). Stimulation of adrenergic pathways activates protein kinases that phosphorylate titin and change its elastic properties, which leads to changes in stiffness at the myocardial level (Kruger and Linke, 2006; Hidalgo et al., 2009; Kruger et al., 2009). Titin-based passive stiffness affects the pressure-volume relationship of the beating heart and can be a direct cause of diastolic dysfunction (Chung and Granzier, 2011). Changes in titin isoform expression and phosphorylation modulate titin-based stiffness in a sarcomere length dependent manne (Fig. 1C), and the presence of multiple mechanisms that alter titin elasticity suggests that fine-tuning titin stiffness is physiologically relevant.

Differential expression of titin isoforms significantly impacts titin-based passive tension because of the intimate relationship between the size of titin's I-band region and the force needed to extend it. Three classes of cardiac titin isoforms exist: N2B, N2BA and fetal cardiac titin (FCT) (Fig. 1B). The primary difference between these three groups is the size of their extensible segments; the regions of titin that are necessary for binding to the Z-disk, thin filament, thick filament, and M-band are largely identical (Labeit and Kolmerer, 1995). Since titin elasticity is primarily defined by its I-band region, the isoform-specific identification of I-band composition has been heavily researched, and numerous splicing pathways have been identified (Lahmers et al., 2004; Greaser et al., 2005). It is now clear that the N2B isoform has the shortest I-band region (and is therefore most "stiff"), FCT isoforms have the longest I-band region (most compliant), and N2BA titin is intermediate (Fig. 1C). The passive tension-sarcomere length relationship of the N2B, N2BA, and FCT isoforms is welldescribed by treating the respective I-band regions as molecular (entropic) springs of varying extensibility, and an in-depth understanding of the origin of titin-based passive tension is warranted.

\section{Origin of titin-based passive tension}

\subsection{Entropic force}

The passive tension generated when striated muscle is stretched comes from titin and the extracellular matrix (ECM) (Wu et al. 2000). The two types of resistance are different, however, as the ECM contribution is an elastic force derived primarily from collagen's high tensile strength and intertwined matrix orientation (Weber, 1989), while titin-based passive tension is entropic in nature. This entropic force is due to titin's extensible I-band region that contains three distinct spring-like elements. The I-band region of titin is unconstrained in the sarcomere (i.e. not attached to actin or myosin) and freely interacts with the thermal energy of the surrounding aqueous medium. The thermal energy at room (and body) temperature is high enough that protein structure is constantly changing as a protein explores all the conformations accessible at that given temperature. The sum of all configurations a protein can occupy determines the phase-space volume of that protein, with each possible configuration of atomic coordinates and momenta corresponding to a unique point, or microstate, in the phase-space. Important to note is that each microstate has an associated probability of occupancy $p_{i}$ and energy $E_{i}$, and that in solution an ensemble of proteins occupies various microstates according to the probability of occupancy (note, microstates with equal $E_{i}$ have equal $p_{i}$ ). Consider the freely-jointed chain, a 3D random walk of monomers of fixed length $L$ (Fig. 2A), which is a simple discrete model to describe an unstructured protein. Note that as $L \rightarrow 0$ the freely-jointed chain becomes continuous (wormlike chain; Fig. 2B). There is no interaction between the monomers, which means all configurations have the same energy. Therefore, all configurations are equally likely and the chain freely diffuses through different microstates as time progresses (for proteins, this diffusion is due to thermal energy). However, only one microstate (configuration) describes the chain being held fully-taut from end to end while numerous microstates are associated with the chain occupying a compact configuration. Because all microstates are occupied with equal probability, it is equally like that the fully-taut configuration and a single compact configuration will be occupied. But since there are vastly more compact configurations, probability alone dictates that the chain will almost always exist in a compact state. Now, lets consider the PEVK element and the unique sequence of the N2B element that are thought to occupy a largely random coil structure, as evidenced by single molecule force spectroscopy experiments that measured smooth (absence of unfolding events) force-extension profiles for both (Li et al., 2001; Watanabe et al., 2002b; Leake et al., 2006; Anderson et al., 2010). In an aqueous environment, these unstructured proteins tend to occupy a compact configuration (small radius of gyration) (Fig. 2C) because there are many more compact microstates compared to extended microstates. To increase the end-to-end length of the protein a force needs to be applied on the ends of the molecule to "bias" the extended conformations and reduce the conformational entropy (randomness) of the chain. The molecular force that resists the external force applied to extend the molecule is the entropic force generated by the random coil protein, which physically results from diffusion that tends to keep the protein compact. If the external force is removed, the protein will quickly re-assume a compact configuration because of this apparent entropic force, which, physically, is powered by diffusion. This entropic restoring force is precisely what is measured in single molecule experiments that stretch random coil proteins (although bond stretching becomes relevant at high fractional extensions) and is the basis for titin-based passive tension in the cardiac cell (Leake et al., 2006; Anderson et al., 2010).

\subsection{Wormlike chain equation}

The force needed to extend a polymer (or random coil protein) is well-described by the wormlike chain (WLC) equation (Marko and Siggia, 1995). The WLC equation is an approximate interpolation formula that describes the non-linear force needed to fractionally extend a polymer of given stiffness and length:

$F=\frac{k_{\mathrm{B}} T}{L_{\mathrm{p}}}\left(\frac{z}{L_{\mathrm{c}}}+\frac{1}{4\left(1-z / L_{\mathrm{c}}\right)^{2}}-\frac{1}{4}\right)$

$F$ is the force, $k_{\mathrm{B}} T$ is thermal energy, $L_{\mathrm{p}}$ is the persistence length of the molecule, $L_{c}$ is the contour length of the molecule (the distanced traced along the molecular backbone), and $z$ is the endto-end length of the molecule (the Euclidean distance between the molecule termini). The persistence length quantifies the "stiffness" of the molecule, or how easily the molecule bends; it defines the distance along the backbone of the molecule that vectors tangent to the backbone are correlated (Fig. 2B), and it is routinely used to quantify an unstructured protein's resistance to stretch. Qualitatively, a lower $L_{\mathrm{p}}$ describes a more flexible molecule. Note, however, that according to the WLC equation a low $L_{\mathrm{p}}$ is associated with a high force needed to stretch the polymer, which is counterintuitive considering that stiffness and force are proportional $(|F|=k x)$ in mechanical springs. This microscopic phenomenon is readily understood when considering entropy. A more flexible molecule can occupy a compact structure in more ways 
A

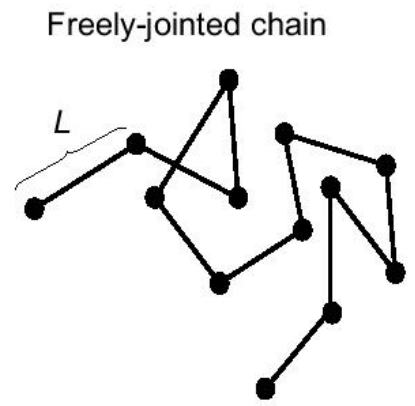

B

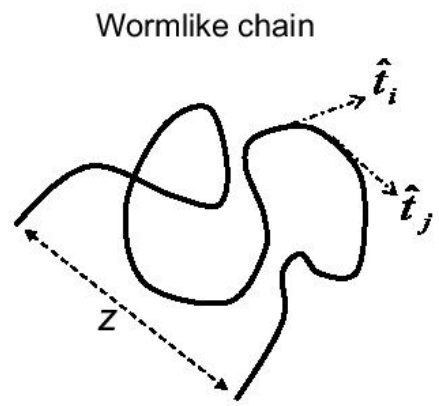

C

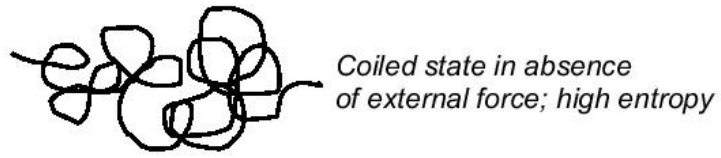

Force biases an extended

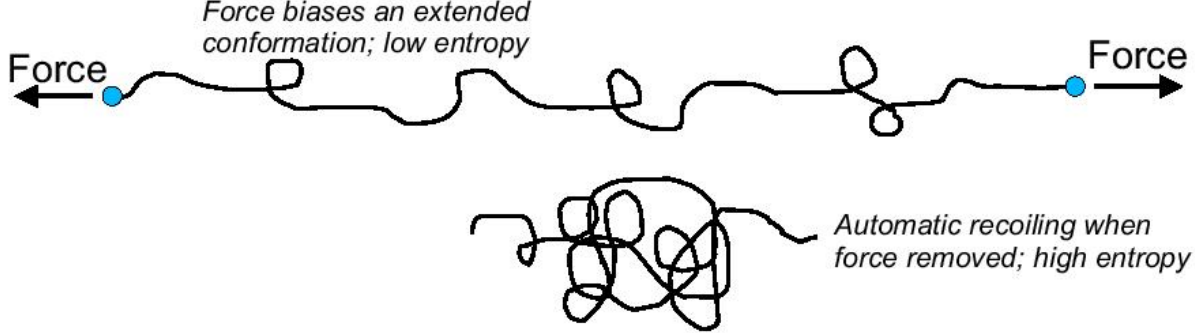

Fig. 2. Simple models of random coil proteins. A) The freely-jointed chain is a 3D random walk of rigid monomers of length $L$ and B) the wormlike chain model represents a continuous, homogenous polymer. Both chains can occupy numerous conformations at low fractional extensions (end-to-end distance $z<<$ molecular contour length) but only one conformation when $z=L_{c}$. C) External force is required to extend a flexible polymer. The molecular restoring force than opposes the external force is the entropic force generated by the polymer. This entropic force is powered by diffusion and is the apparent force that recoils the protein when external force is removed.

(more microstates) than a more rigid molecule; therefore, there is a stronger entropic force that biases the molecule to exist in a compact state compared to an extended state, and hence a larger external force is needed to overcome this entropic force to increase the end-to-end length (stretch) of the molecule. Persistence length is defined for an ideal, homogenous polymer and therefore is only an approximation of the behavior of a random coil protein. Regardless, the $L_{\mathrm{p}}$ of a molecule is often determined by fitting experimental force-extension data to the WLC equation (Fig. 4C) and is very useful for summarizing the force-extension behavior of extensible proteins. The WLC equation also shows why N2B titin is the stiffest isoform: at a given sarcomere length the I-band regions of all titin isoforms have the same end-to-end length $(z)$, but because the I-band region of the N2B isoform has the shortest contour length its fractional extension $\left(z / L_{C}\right)$ is greater than the N2BA and FCT isoforms and, according to WLC equation, requires more force to stretch.

\section{Modulating titin stiffness}

The two most recognized mechanisms for tuning titin-based passive tension are changes in isoform expression and posttranslational modifications of titin. Isoform changes affect titin stiffness because the cardiac titin isoforms (N2B, N2BA, FCT) contain I-band regions of different length that are generated by titin splicing pathways.

\subsection{Isoform splicing}

The specific I-band compositions of titin isoforms is processed by complex and poorly-understood pre-mRNA splice pathways that result in varying degrees of titin stiffness. In humans, the titin gene is located on chromosome 2 , contains 363 exons, and has a maximal coding capacity of 38,138 residues (4.2 MDa) (Bang et al., 2001). Splicing events result in titin isoform expression being muscle specific (Wang et al., 1991; Labeit and Kolmerer, 1995), and three classes of isoforms are found in the heart: adult N2B and N2BA isoforms and fetal cardiac titin (Lahmers et al., 2004; Greaser et al., 2005). The I-band region of the titin gene comprises $\sim 220$ exons (Bang et al., 2001), with a subset of these exon gene products constitutively expressed in all cardiac isoforms (Fig. 3): the proximal tandem Ig segment (Ig1-15; some Ig domains coded by two exons), the N2B element (containing Ig24-26 and a 572 residue unique sequence), Ig27, the PEVK sequence encoded by exons 219225, and the distal tandem Ig segment (Ig84-105). These domains are found in the I-band regions of all cardiac isoforms and are the entire I-band of the $2970 \mathrm{kDa}$ N2B isoform (Fig. 1B). The N2BA isoform also contains a variable number of Ig domains in the socalled middle tandem Ig segment (Freiburg et al., 2000), the N2A element (which contains unique sequences and Ig80-83), and additional PEVK sequence (Freiburg et al., 2000). The additional Iband elements in N2BA titin make it larger ( $23.3 \mathrm{MDa})$ and more compliant than the N2B titin isoform. In adult left ventricle (LV), 


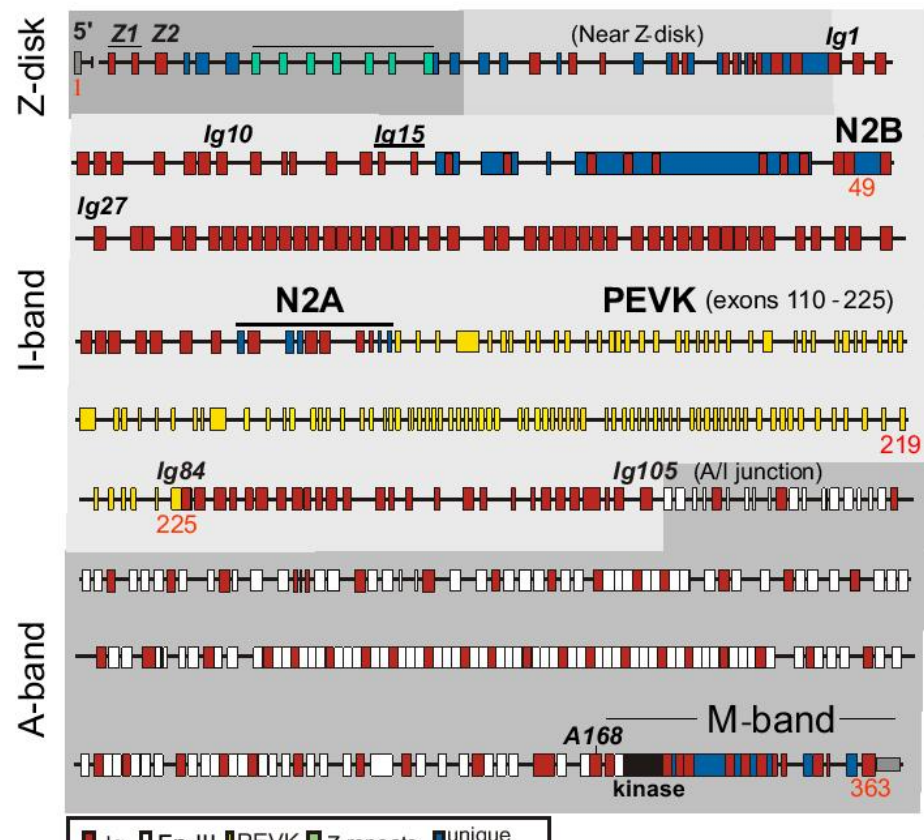

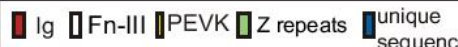

Fig. 3. The exon structure of the human titin gene (based on (Bang et al., 2001)). Titin contains 363 exons, $\sim 220$ of which are found in the I-band region. Titin's I-band region comprises immunoglobulin(Ig)-like domains, the PEVK element, and unique sequences. The thick filament bound A-band region of titin contains Ig and fibronectin-III (Fn-III) domains exclusively. Domains and exons alluded to in the text are labeled.

N2B titin is more abundant than N2BA ( $~ 80 \%$ of mouse titin and $\sim 60 \%$ of human titin (Cazorla et al., 2000)), although in certain disease states these ratios are different. The third class of cardiac titin isoforms is the fetal cardiac titins (FCT; 3.5-3.6 MDa (Lahmers et al., 2004)) that express additional Ig domains in the middle tandem Ig segment and the longest PEVK sequence (Opitz et al., 2004; Greaser et al., 2005). FCT isoforms dominate at birth but are replaced by the shorter, stiffer adult isoforms during neonatal development (Lahmers et al., 2004). Titin isoform expression occurs at the level of the half-sarcomere (Trombitas et al., 2001) and the mechanics of each isoform depend on the size of its I-band region, with shorter I-band regions corresponding to higher titinbased passive tension. Titin isoform expression differences are responsible for myocardial passive tension variability across species, between muscle types, during development, and throughout disease phenotypes. For example: smaller mammals typically have faster heart rates, which correlates with stiffer myocardium and a lower N2BA:N2B expression ratio (Cazorla et al., 2000), skeletal muscle titin isoforms vary widely between muscle types and are much longer than cardiac isoforms (Neagoe et al., 2003), the large FCT cardiac isoforms are present in neonates but are replaced by N2B and N2BA during development (Lahmers et al., 2004), and cardiac titin isoform expressions are different in certain disease states of dog, rat, and humans (Neagoe et al., 2002; Wu et al., 2002; Warren et al., 2003; van Heerebeek et al., 2006). One hypothesis regarding the functional significance of changes in titin isoform expression is that the ratio of N2BA:N2B titin helps set an animal's optimal heart rate. For example, high levels of N2B, which results in stiffer myocardium, allows for rapid early diastolic filling and rapid setting of the end diastolic volume, both of which are necessary for the short diastolic filling times associated with high heart rates. This suggests that cardiac titin expression may help define the optimal heart beat frequency and that post-translational modifications, which can rapidly alter titin stiffness, may couple myocardial stiffness with acute cardiac demand.

Of particular interest to researchers is how titin splicing is altered by hemodynamic demands on the heart. For example, a dilated cardiomyopathy (DCM) model was induced in dogs by imposing pacing tachycardia for two weeks that resulted in chamber dilation and increased chamber stiffness (Bell et al., 2000) and a study in which pacing was administered for four weeks showed down-regulation of compliant N2BA titin and upregulation of stiff $\mathrm{N} 2 \mathrm{~B}$ titin in addition to, as expected, increased titin-based passive tension (Wu et al., 2002). A hypertensive rat model subject to pressure overload also showed reduced N2BA isoform expression (Warren et al., 2003), which is consistent with the elevated passive tension found in hypertensive rat cardiomyocytes (Conrad et al., 1995; Cicogna et al., 1999).

In humans, patients with coronary artery disease (CAD) exhibited increased N2BA expression that accompanied decreased myofibrillar stiffness, although LV wall stiffness was higher in CAD patients and explained by increased collagen and desmin expression levels (Neagoe et al., 2002). Unlike the animal models mentioned above in which increases in titin-based stiffness contributed to the disease pathology, it seems that decreased titin stiffness in human CAD patients acts to compensate for the increased ECM-based stiffness that accompanies the disease (Porter and Turner, 2009). In patients with end-stage heart failure due to 
DCM, N2BA:N2B ratio was increased and corresponded with lowered passive stiffness (Nagueh et al., 2004). In addition, the physiological relevance of changes in titin isoform expression was shown by positive correlations between N2BA:N2B ratio and chamber compliance parameters, which suggests that DCM affects diastolic filling by lowering titin-based stiffness (Nagueh et al., 2004). Interestingly, the same study showed an increased N2BA:N2B ratio also correlated with improved exercise tolerance, which suggests that the titin isoform shift may be a beneficial adaptation in response to heart dilation. The work on animal models subject to short-term cardiac stress showed that changes in titin isoform expression contributed to the pathology, while chronic cardiac stress in human patients showed adaptation in titin expression to try to compensate for deleterious changes in the other determinant of myocardial stiffness (ECM). The relationship between cardiac insult and changes in titin is clearly complex, and a better understanding of titin is needed to more effectively confront cardiac disease.

\subsection{Post-translational modifications}

Changes in titin isoform expression occur on the time scale of days to weeks depending on the stage of development and species (Wu et al., 2002; Lahmers et al., 2004), but adjustment of titin's contribution to passive tension can also occur rapidly via posttranslational modifications (PTMs) of titin's I-band region. Cyclic AMP (cAMP)-dependent protein kinase A (PKA) is activated by $\beta$ adrenergic stimulation and has been shown to phosphorylate the unique sequence of the cardiac specific N2B element (N2B-Us). Phosphorylation by PKA reduced passive tension in skinned rat cardiomyocytes (Yamasaki et al. 2002) and human cardiac fibers (Kruger and Linke, 2006). The effect in human fibers was more pronounced when de-phosphorylation by protein phosphatase- 1 (PP1) was performed before PKA treatment, which suggests that basal levels of phosphorylation play an important role in determining titin-based passive tension.

Protein kinase G (PKG), which is cGMP-dependent, also phosphorylates the N2B-Us and reduces passive tension, and the site of phosphorylation is the same residue that PKA targets (Kruger et al., 2009). Passive tension of skinned fiber bundles from human LV was significantly reduced only after PP1 treatment removed basal phosphorylation levels, which, similar to the PKA results, suggests that basal levels of titin PTMs play important roles in vivo. A single molecule study on N2B-Us treated with PKG suggested that phosphorylation increases the $L_{\mathrm{p}}$ of the N2B-Us (Kruger et al., 2009), which corresponds to a reduction of passive tension at the tissue level. It is worth noting that the decrease in passive tension seen at the tissue level is similar to the change in tension that would result from an increased N2B-Us $L_{\mathrm{p}}$ following PKG treatment. Because single molecule experiments are able to quantify the effect of a treatment on the mechanical properties of I-band elements, it can be surmised if the effect at the tissue level is fully explained by changes at the molecular level or if a more dynamic hypothesis is needed to explain how the treatment variable affects changes in cardiac stiffness. However, more single molecule measurements are needed to strengthen this result because of the limited data gathered in the initial study.

Most recently, it has been shown that protein kinase C (PKC) specifically phosphorylates titin at two highly-conserved serine residues in the constitutive PEVK element found in all cardiac titin isoforms (Hidalgo et al., 2009). PEVK phosphorylation by $\mathrm{PKC} \alpha$ (the predominant isozyme in the heart and an important player in contractile dysfunction and heart failure (Molkentin and Dorn, 2001; Belin et al., 2007)) increases titin-based passive tension, an effect that is also exacerbated by PP1 pre-treatment. Following
PKC $\alpha$ phosphorylation, PP1 treatment reversed the passive tension increase (Hidalgo et al., 2009), which suggests that $\mathrm{PKC} \alpha$. phosphorylation directly influences titin stiffness. In addition, single molecule experiments have shown that phosphorylation of PEVK lowers the $L_{\mathrm{p}}$ of PEVK in a site-specific manner (Anderson et al., 2010). This increased flexibility results in an entropically stiffer molecule that is predicted to increase titin-based passive tension to a level similar to what was found in skinned myocardium (Hidalgo et al., 2009). Further solidifying the link between PKC $\alpha$. phosphorylation and PEVK, it was shown that PKC $\alpha$ had no effect on the passive stiffness of mice missing the constitutive PEVK element (PEVK KO) (Hudson et al., 2010). These results, which span from the single molecule level to the tissue level, show that PKC $\alpha$ phosphorylation directly influences titin-based passive tension. This opens the possibility of using $\alpha 1$-adrenergic antagonists, which would minimize PKC $\alpha$. activity, as a potential therapy for cardiomyopathies characterized by increased myocardial stiffness.

Because titin can be phosphorylated by various protein kinases resulting in disparate effects, elucidating the phosphorylation status of titin is important when a comprehensive understanding of titin-based stiffness is desired. For example, in rodents PKA reduces passive tension by approximately $30 \%$ (depending on sarcomere length (Yamasaki et al., 2002)) while PKC increases passive tension by $30 \%$ (Hidalgo et al., 2009). These kinase effects can also be titin isoform dependent (Fukuda et al., 2005). A thorough analysis of titin isoform expression and PTMs must be performed when deconstructing passive tension determinants in healthy and diseased myocardium.

\section{Studying physical properties of titin I-band elements}

In order to gain a better fundamental understanding of how the spring-like elements of titin's I-band region contribute to titinbased passive tension and how phosphorylation of the spring-like elements leads to changes in titin stiffness, the physical properties of the PEVK, N2B, and tandem Ig domains have been studied in isolation using single molecule techniques. Although laser tweezers are an excellent tool for probing the biomechanics of single molecules, most single molecule titin studies have utilized the atomic force microscope because it can easily access high force regimes (to elicit Ig unfolding), stretch short molecular fragments without the need for long linkers (as required for laser tweezers), and generate multiple molecular force-extension traces quickly.

\subsection{Atomic force microscopy}

Direct measurement of the physical properties of single molecules requires force and length resolution on the order of piconewtons and nanometers, respectively. Because it is versatile and relatively simple to use, the atomic force microscope (AFM) (Binnig et al., 1986) has been the single molecule force spectroscopy choice for many. Although AFMs are also widely-used for surface imaging ( $x y$ plane scanning), its ability to stretch proteins in the $z$-direction (normal to a flat surface) is ideally-suited for high throughput generation of molecular force-extension curves (for a recent review on AFM and titin see (Linke and Grutzner, 2008)). The basic AFM protocol for stretching protein fragments is as follows (Fig. 4). Purified recombinant protein is spotted onto a microscope slide or coverslip and allowed to adsorb onto the surface. The AFM cantilever tip then probes the protein-coated surface vertically until an adsorbed molecule attaches to the tip (a non-specific interaction unless the cantilever has been functionally modified). This tethered protein is then stretched as the tip retracts from the surface. The force that develops in the molecule is measured by the deflection of a focused light source that reflects off the back of a cantilever tip of 
A
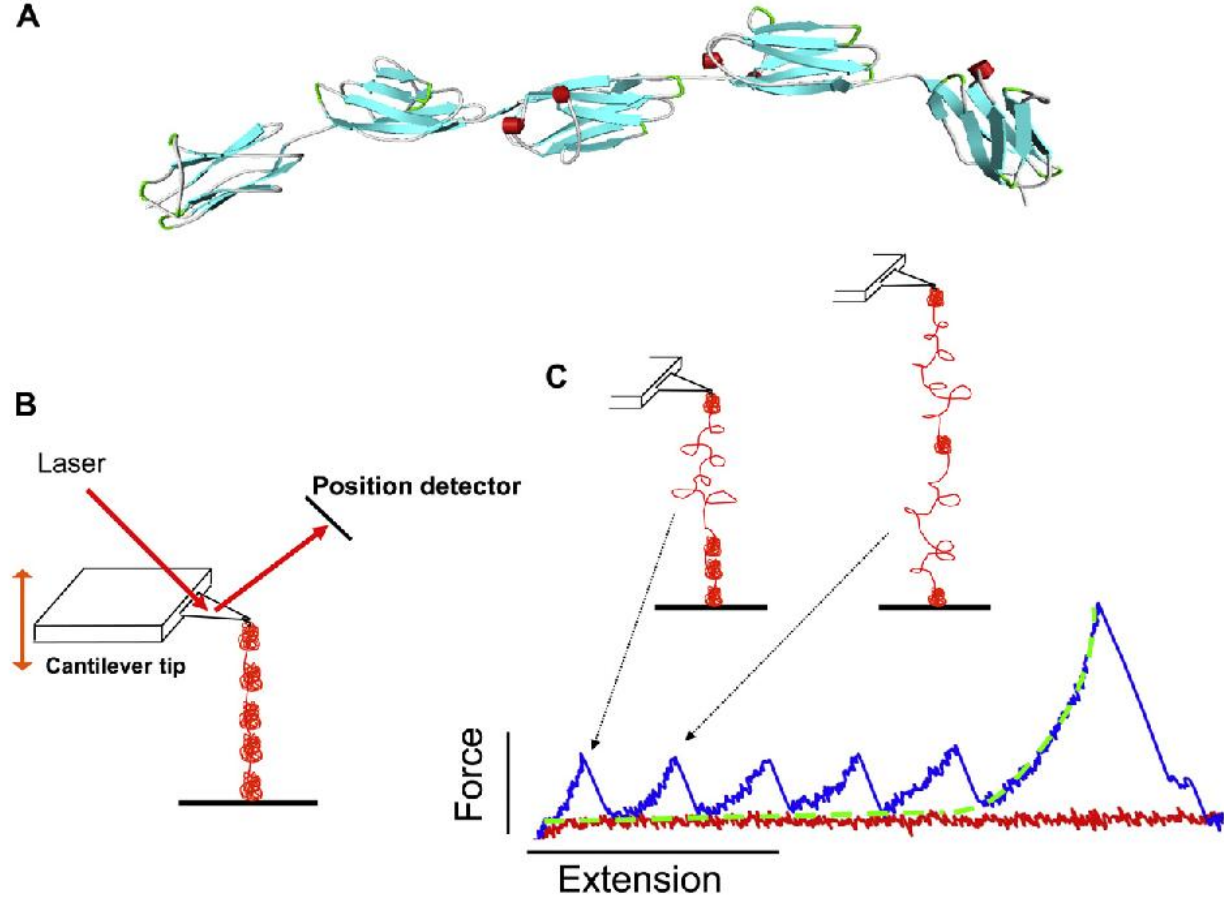

Fig. 4. A) A ribbon schematic of five serially-linked Ig domains. $\beta$-strands are colored blue and alpha helices are red. The atomic coordinates were downloaded from the Worldwide Protein Data Bank, PDB ID: 3 B43 (von Castelmur et al, 2008). Each folded Ig domain is 4-5 nm in diameter and connected by short linker sequences. B) Simplified AFM schematic. The cantilever tip probes a protein-coated surface until a molecule is tethered. The molecule is then stretched and the force that develops is determined by measuring the deflection of the laser off the cantilever. C) A tethered polyprotein is stretched to induce domain unfolding. Large structural transitions, such as complete unfolding of an Ig domain, result in a large contour length increase, a release of tension in the molecule, and a sharp unfolding force peak. In the example shown, the molecule is still attached after all five domains unfold; the last force peak is due to stretching the completely unfolded peptide that behaves as an entropic spring and is well-described by the WLC equation (fitted dashed line),

known stiffness. The force needed to stretch the molecule is equivalent to the force needed to bend the cantilever tip. Consequently, the force needed to stretch the molecule as a function of cantilever position (which can be converted to molecular end-toend length, $z$ ) is measured to generate a force-extension trace. The force that acts on the molecule increases as the extension increases, and this process continues until a force-induced structural transition is realized. When stretching serially-linked Ig domains, for instance, this structural transition is the complete unfolding of a single Ig domain from its native, globular structure to an unfolded, random coil structure. This sudden increase in contour length removes the tension in the tethered molecule and is recorded as a clearly-identifiable unfolding force peak. Unstructured proteins, on the other hand, are mechanically unstable and do not exhibit large unfolding force peaks when stretched. In this case, AFM traces of flexible proteins are fit with the WLC equation to estimate an effective persistence length of the protein; this is a convenient way of quantifying the force needed to extend a flexible protein.

\subsection{PEVK}

Appropriately named due to its high density of proline (P), glutamate (E), valine (V), and lysine (K) residues, the PEVK element of titin's I-band region behaves as an entropic spring. Although some local secondary structure in the form of polyproline II helices has been suggested (Ma et al., 2001), long range cooperativity between the helices is apparently absent (Gutierrez-Cruz et al. 2001), and polyproline helix structure is uncommon in the PEVK element of the cardiac N2B isoform (Watanabe et al., 2002b). It is worth noting that the N2BA titin isoform contains two polyE (negatively-charged glutamate-dense regions) and 20 PEVK repeat motifs (26-28 residues), compared to zero and five, respectively, found in the N2B isoform (Greaser, 2001). Therefore, it cannot be assumed that the properties of PEVK are identical in N2B and N2BA titin, although most research (such as that cited in this review) has studied the constitutive PEVK sequence that is the entire PEVK element of the N2B titin isoform because all mammalian titin isoforms contain these PEVK exons. The PEVK element found in the $\mathrm{N} 2 \mathrm{~B}$ isoform is 188 residues in length (assuming a random coil and maximal residue spacing of $0.38 \mathrm{~nm} /$ aa gives a $L_{c}$ of $71 \mathrm{~nm}$ ) while the N2BA isoform has a $\sim 800$ residue PEVK element $(\sim 300 \mathrm{~nm})$. The absence of large stable structures in PEVK is suggested by the high concentration of proline residues and charge clusters (Labeit and Kolmerer, 1995) and is evidenced by smooth force-extension traces that also lack hysteresis (Li et al., 2001; Watanabe et al., $2002 \mathrm{~b}$ ). The persistence length of PEVK is $\sim 1 \mathrm{~nm}$ (Li et al., 2001; Linke et al., 2002; Watanabe et al., 2002b; Anderson et al., 2010), but evidence suggests that it is not purely a random coil. Phosphorylation of PEVK by protein kinase $\mathrm{C}$ and mutation of the phosphorylated residues (S26 and S170) both reduce the apparent $L_{\mathrm{p}}$ of PEVK, which suggests that these residues are involved in 
a structural network with other amino acids (Anderson et al., 2010) because a simple mutation would not significantly change the $L_{\mathrm{p}}$ of a pure random coil. However, primary sequence does have some effect on elasticity (Cheng et al., 2010). In addition, the PEVK sequence around S26 and S170 is highly conserved, which also suggests some PEVK structure (Hidalgo et al., 2009), although as mentioned previously this structure must not be mechanically stable since AFM force-extension traces do not show unfolding force peaks. Although the PEVK exhibits a smooth force-extension trace that can be fit relatively well with the WLC equation to determine an "effective" $L_{p}$ of the molecule, the PEVK element (like all biomolecules) is not homogenous and parameters used to describe the entire protein are approximations.

\subsection{N2B-Us}

The cardiac-specific N2B element is found in both the N2B and N2BA titin isoforms of the heart. It contains three Ig domains (Ig 24-26) and a 572 residue unique sequence (N2B-Us) that, like the PEVK, behaves as an entropic spring. If we assume a pure random coil, the $L_{c}$ of the N2B-Us is $217 \mathrm{~nm}$ (572 aa $\times 0.38 \mathrm{~nm} / \mathrm{aa}$ ). AFM experiments have found the $L_{\mathrm{p}}$ of the N2B-Us to be $\sim 0.65 \mathrm{~nm}(\mathrm{Li}$ et al., 2002; Watanabe et al., 2002b; Zhu et al., 2009), which makes it more flexible (and harder to stretch) than the PEVK element. Also similar to the PEVK, the force-extension profile of the N2B-Us does not contain discernable force peaks or hysteresis (Watanabe et al., 2002b). Since the force resolution of most AFMs is 5-10 pN, low-force structural transitions cannot be excluded. Due to its presumably random coil conformation, the N2B-Us structure has not been solved, which makes structural transitions due to ligand binding (Zhu et al., 2009) and phosphorylation (Leake et al., 2006; Kruger et al., 2009) impossible to predict. This same difficulty is present when trying to predict the physical mechanism that results in changes in PEVK properties following phosphorylation (Anderson et al., 2010).

\subsection{Tandem Ig segments}

Titin's I-band region contains serially-linked immunoglobuli$\mathrm{n}(\mathrm{Ig})$-like domains that have a $\beta$-barrel fold characteristic of the intermediate I-set of the immunoglobulin superfamily (Pfuhl and Pastore, 1995) (Fig. 4A). The proximal tandem Ig segment near the Z-disk contains 15 Ig domains and the distal tandem Ig segment near the A-band contains 22 domains. Ig domains also flank the N2B-Us and PEVK element (Fig. 1B). Unlike the N2B-Us and PEVK, Ig domains have well-defined structures that have been solved using NMR, X-ray crystallography, and small angle X-ray scattering techniques (Improta et al., 1996; Mayans et al., 2001; von Castelmur et al., 2008; Stacklies et al., 2009). This has allowed for molecular dynamics experiments to study Ig domain dynamics at the atomic level (Lu et al., 1998; Lee et al., 2010), and, consequently, much more is known about the Ig domains than the PEVK and N2B-Us. AFM work on titin Ig domains is extensive and has shown that I-band Ig domains are mechanically very similar, although Ig unfolding force is domain-dependent (Marszalek et al., 1999; Li et al., 2000; Fowler et al., 2002; Watanabe et al., 2002a). Connected in series like beads on a string, globular Ig domains are $4-5 \mathrm{~nm}$ in diameter and are separated by short ( $2-4$ residues) amino acid linkers (Marino et al., 2005). When stretched with an AFM, the domains first become taut while remaining in their folded state. When enough tension develops in the tandem protein, one of the Ig domains pops open, rapidly transitioning from its $\beta$-barrel structure to a fully unfolded (random coil) peptide chain. Ig domains comprise $\sim 80-90$ amino acids (on average) which results in a $\sim 30 \mathrm{~nm}$ contour length increase following total unfolding. This sudden increase in contour length removes the tension in the polyprotein and results in a large force peak in the force-extension profile (Fig. 4C). Of particular interest to researchers is the force needed to unfold Ig domains and their kinetic rates of unfolding and refolding; knowing these parameters allows for prediction of how frequently Ig domains unfold, which is important for determining titin-based passive tension. Tandem Ig domains are physically not well described by the WLC equation because they are not random coils in their native state and they require much less force to extend when in their folded structure (for example, the effective $L_{\mathrm{p}}$ of folded Ig domains has been estimated at $10 \mathrm{~nm}$ (Granzier and Labeit, 2005), which is reasonable considering that adjacent Ig domains must be correlated in orientation due to their short linker sequences). However, unfolded Ig domains are random coils that behave as entropic springs and would more significantly contribute to passive tension provided that they unfold under physiological forces. In AFM experiments, Ig domains typically unfold at forces between 150 and 250 pN (Li et al., 2000; Watanabe et al., 2002a; Zhu et al., 2009) (unfolding force is velocity dependent, see below), but in the sarcomere the force felt by each titin I-band at end diastole is estimated to be $<5 \mathrm{pN}$ when considering muscle mechanics (Nedrud et al., 2011), thick filament density (Irving et al., 2011), and working sarcomere length (Chung and Granzier, 2011) data.

Single molecule studies on Ig dynamics have also been used to elucidate the link between titin and cardiac disease. Recently, a mutation in the 10th Ig domain in the proximal tandem Ig segment has been linked with arrhythmogenic right ventricular cardiomyopathy (ARVC) (Taylor et al., 2011), which is characterized by fibrofatty replacement of the myocardium and an increased risk of cardiac arrhythmias and sudden death. It is surprising that a single point mutation in an Ig domain seemingly leads to cardiomyopathy, but the combination of a variety of experimental techniques has suggested a hypothesis that links altered Ig10 dynamics with degradation of healthy myocardium. Nuclear magnetic resonance (NMR) data and proteolysis assays have shown that Ig10 domains harboring the disease-linked mutation are structurally compromised and more prone to degradation (Taylor et al., 2011); AFM data also shows that mutant Ig10 unfolds at a lower force compared to native $\operatorname{Ig} 10$ (Anderson et al., in press), which is consistent with the idea that the mutation weakens the domain's $\beta$-barrel structure and results in a higher percentage of unfolded mutant Ig10 compared to native Ig10. This propensity to exist in an unfolded structure combined with the increased rate of degradation suggests that the mutation leads to cleavage in titin's I-band region, which would abolish titin's essential force-generating mechanism and likely lead to accelerated titin turnover and possibly even apoptosis. Clearly more work is needed to develop this hypothesis, but these interesting ideas are made possible because of the ability to study individual titin components on the single molecule level.

\subsubsection{Analysis of Ig unfolding}

Substantial AFM work has investigated the properties of titin's Iband region, in particular the forced unfolding of Ig domains. Two phenomena related to Ig unfolding have been identified: the dependence of unfolding force on unfolding peak number and the dependence of unfolding force on pulling velocity. The conceptual ideas that explain these phenomena are worth discussing because they offer valuable insights regarding the dynamics that govern titin's I-band behavior. Furthermore, proper analysis of the dependence of unfolding force on peak number is needed to obtain accurate data, although only recently has theory been applied to accomplish this task.

In order to determine the force needed to unfold a globular domain, it is best to stretch a homopolyprotein ( $\mathrm{N}$ identical domains connected in series). If a heteropolyprotein is stretched, it 
is impossible to assign unfolding force peaks to particular domains in the series because each domain unfolds stochastically and independently. However, if every domain in the series is the same, each unfolding force peak represents unfolding of the domain of interest. Previous studies cleverly used homopolyproteins to determine the properties and kinetic rates of Ig domains but usually combined all unfolding force data during analysis (i.e. the first unfolding force peak was not distinguished from the last unfolding force peak). This procedure assumes that the unfolding forces obtained from unfolding a tandem protein and a single protein are sampled from the same distribution, but this has been shown to be false (Zhmurov et al., 2010). Instead, the unfolding force depends on the number of domains being stretched, which leads to the first unfolding force of a force-extension trace being lower than the second peak, the second being lower than the third, and so on. This peak dependence of unfolding forces is a combinatorial phenomenon, and a method based on order statistics theory has been developed to remove this peak dependence and allow for unfolding force measurements to be combined when analyzing data (Zhmurov et al., 2010), which allows for more robust fitting procedures. This theory was published only recently, which explains why most unfolding force data of homopolymeric proteins in the literature is analyzed with all unfolding force peaks combined (Li et al., 2000; Watanabe et al., 2002a). Conceptually, it is simple to understand why unfolding force increases with peak number. Consider a string of five identical Ig domains held taut in solution. Due to thermal energy one of the domains will unfold first, say at time $t_{1}$, and let us assume no refolding. A short while later another domain unfolds, say at $t_{2}$, and this process continues for $t_{3}, t_{4}$, and $t_{5}$. Now consider a string of 100 identical Ig domains and the subsequent $t_{1}$ ' through $t_{100}$. Because there are more Ig domains in this long string, the first Ig domain that stochastically unfolds will unfold, on average, quicker than the first domain that unfolded in the five Ig chain. In other words, $t_{1}{ }^{\prime}<t_{1}$ because more domains had the potential to unfold in the longer Ig chain. Now consider an AFM pulling experiment with, for example, ten identical domains linked in series. All domains are folded prior to stretching and force develops in the molecule as the cantilever tip pulls away from the slide surface, with a force peak generated each time a domain unfolds. Note, however, that before the first force peak was generated ten domains had the potential to unfold, but before the final (10th) force peak only one domain had the potential to unfold. As mentioned above, the time that elapses before the firs unfolding event takes place $\left(t_{1}\right)$ is shorter when ten domains are folded than when only one domain is folded. Therefore, the unfolding force of peak one is less than peak ten. From this same logic, it follows that $F_{1}<F_{2}<\cdots<F_{\mathrm{N}}$ on average for a serially-linked homopolymer of length $N$, which is what has been shown theoretically (Zhmurov et al., 2010) and experimentally (Anderson et al., in press).

Unfolding force also depends on the pulling velocity of the AFM tip (Li et al., 2000; Watanabe et al., 2002a), with higher pulling velocities resulting in larger unfolding forces, and intricate theories have been developed to extract kinetic information from data gathered at wide-ranging pulling speeds (Hummer and Szabo, 2003; Dudko et al., 2008). Consider a serially-linked Ig homopolyprotein. Although the $\beta$-barrel structure imparts high stability to Ig domains, these domains still unfold under no external force with some finite probability (typically $\sim 10^{-6}-10^{-4} \mathrm{~s}^{-1}$ ( Li et al., 2000; Watanabe et al., 2002a)) due to thermal fluctuations. In an AFM pulling experiment, the domains are mechanically stretched which strains bond networks (hydrogen, disulfide, etc.) until the protein eventually denatures. For Ig domains, it has been shown that after the main rupture (force peak in AFM traces) the rest of the molecule unravels at a much lower force (Lu et al., 1998), which results in a single unfolding force peak being measured, although unfolding intermediates have also been seen (Marszalek et al., 1999). To understand why higher pulling speeds result in higher unfolding forces, consider the mechanical and thermal energy contributions. Both of these forms of energy can unfold the protein when the molecule is being stretched. When the stretch speed is fast, the force loading rate (N/s) acting on the molecule is increased relative to a slower stretch speed while the thermal energy bombarding the molecule is unchanged. This means that the energy that unfolds the molecule has a larger mechanical contribution than when the stretch speed is slow (i.e. when there is more time for diffusive thermal energy to unfold the protein). Of course, the mechanical force acting on the protein when a domain unfolds is measured by the bending of the AFM cantilever, which is output as the deflection signal (unfolding force peak). In summary, at faster pulling speeds there is less time for thermal energy to contribute towards unfolding the protein and more of a burden on mechanical energy.

\subsubsection{Ig kinetics}

Although it is simple to determine the force needed to unfold titin Ig domains, this information is not particularly useful when determining how Ig domains contribute to titin-based passive tension because the forces experienced by the I-band are $<5 \mathrm{pN}$ while Ig unfolding force at physiological stretch speeds is much higher (150-250 pN depending on specific Ig domain (Watanabe et al., 2002a; Zhu et al., 2009)). More useful parameters for determining the fraction of Ig domains that are unfolded (behaving as force-generating entropic springs) are the kinetic rates of unfolding and refolding. The unfolding rate at zero force $\left(\alpha_{0}\right)$ is the rate at which a protein transitions from a folded to an unfolded state under no external load, and the refolding rate $\left(\beta_{0}\right)$ is the opposite. Unfolding rate is often estimated by comparing the unfolding force distribution from AFM data with Monte Carlo simulations (Kellermayer et al., 1997; Rief et al., 1997; Li et al., 2000) where force-dependent unfolding is assumed to follow Bell's formula (Bell, 1978) $\alpha(F)=\alpha_{0} e^{F \cdot \Delta x / k_{B} T}$ and $\Delta x$ is the distance along the reaction coordinate (i.e. molecular end-to-end length) from the free-energy minimum of the folded state to the transition barrier. For estimating unfolding rate at zero force $\left(\alpha_{0}\right)$ Monte Carlo methods are used to simulate AFM pulling experiments and generate unfolding force distributions for various $\left(\alpha_{0}, \Delta x\right)$ parameter pairs. The unfolding rate of the protein is then estimated to be the rate used in the simulation that best recreated the experimental data. The unfolding rate at zero force is difficult to measure directly using single molecule methods because of experimental limitations. For example, Ig unfolding is rare under no external force $\left(\sim 10^{-5} \mathrm{~s}^{-1}\right)$ and it is unlikely that a measurement of an unfolding event would occur prior to the molecule being stochastically displaced from the cantilever tip. Force-clamp techniques, in which a tethered molecule is held taut at a constant force (Oberhauser et al., 2001), have been used to directly measure force-dependent unfolding rates, although typically the hold forces are much higher than physiological force values (Garcia-Manyes et al., 2007). In this case, the unfolding rate at zero force is then extrapolated from the force-dependent rates by plotting $\ln \alpha(F)$ vs. hold force. Determining $\alpha_{0}$ with more confidence would require $\alpha(F)$ values at forces closer to 0 , although experimental force noise (typically $\sim 10 \mathrm{pN}$ ), thermal drift, and displacement of the molecule from the AFM tip prohibit direct measurement of unfolding rates at physiological force levels. Estimating low force unfolding rates can also be accomplished by assuming $\alpha(F)=\alpha_{0} e^{F \cdot \Delta x / k_{\mathrm{B}} T}$ with $\alpha_{0}$ and $\Delta x$ determined from Monte Carlo simulations.

Low force refolding rate, however, can be directly measured with AFM refolding protocols. First, a tethered multidomain protein is stretched and unfolded although the protein is not detached from 
the slide surface or cantilever tip. Then the molecule is relaxed as the tip hovers above the slide surface. Because the unfolded domains are essentially random coils held at very small fractional extension, there is very low force acting on the molecule at this point. The unfolded domains are allowed to refold as the low force environment is maintained for a set amount of time, and then the molecule is completely stretched and unfolded to generate a full force-extension trace. By counting the number of "overlapping" force peaks one can infer how many domains refolded during the low force hold. From this information, refolding rate is directly determined (Li et al., 2000). In addition to determining how the large tandem Ig segments contribute to titin-based passive tension, measuring the kinetic rates of unfolding and refolding can also be important for quantifying how a mutation affects the structure of Ig domains in the context of identifying disease mechanisms, as was recently demonstrated with $\operatorname{Ig} 10$ and ARVC (Anderson et al., in press; Taylor et al., 2011).

\section{Viscoelasticity}

Titin's I-band region has been treated as a non-linear spring so far, but sources of viscosity also exist within cardiac myocytes. The presence of viscosity creates a phase shift between stress (force) and strain (length) as the muscle is stretched and increases the force needed to stretch the myocardium because viscosity resists motion and must be overcome during sarcomere length changes (de Tombe and ter Keurs, 1992). A schematic of how viscosity is measured is shown in Fig. 5. The viscous modulus, which quantifies a material's resistance to stretch, is calculated as the ratio of the stress amplitude to strain amplitude times the sine of the phase angle difference between the two. The elastic modulus is calculated by replacing $\sin (\theta)$ with $\cos (\theta)$ in the viscous modulus equation. If the stress and strain traces superimpose there is no phase difference and the material is purely elastic. A $90^{\circ}$ phase difference is present in purely viscous materials. In cardiac muscle preparations, the stress leads the strain as a result of viscosity. Recall Newton's equation of motion in one dimension: $F=m a+\gamma v+k x$, where $\gamma$ represents a viscous parameter that resists motion. When stretching muscle, this viscous resistance must be overcome, and the viscous force is largest when the rate of length change (velocity) is maximum. For sinusoidal strain, $\Delta L / \Delta t$ is largest when the strain switches from negative to positive values (i.e. when the sine wave that describes strain crosses the $x$-axis during stretch). The external force that imposes length changes on the muscle tissue must do work to oppose this viscous force. This is why a positive stress value is measured when there is no strain in the prep (Fig. 5B) and why stress leads strain in tissue and cell mechanics: the myocardium is viscoelastic.

The primary source of titin-based viscosity in the cardiac myocyte comes from the interaction between the PEVK element and actin. In-vitro motility assays (Yamasaki et al., 2001; Linke et al., 2002), single molecule studies (Bianco et al., 2007), and myocyte mechanics (Yamasaki et al., 2001) have established that the interaction between PEVK and actin retards filament sliding during systole and diastole. It has been shown that the PEVK-actin interaction is present in all cardiac isoforms and is a source of hysteresis (Fukushima et al., 2010). Recently, studies using PEVK KO mice showed that the PEVK-actin interaction significantly contributes to viscosity in vivo (Chung et al., 2011). The affinity between PEVK and actin is thought to be an electrostatic effect between negatively charged actin (Kabsch et al., 1990) and PEVK. The complete PEVK region found in N2B titin (which is also found in the N2BA isoform) comprises five basic (pI 9-10) 28 residue PEVK repeats (Greaser, 2001). Therefore, at physiological pH cardiac PEVK has a net positive charge that may promote actin interaction. The hypothesis that PEVK-actin interaction is electrostatic is also supported by the fact that the interaction is dependent on ionic strength (Yamasaki et al., 2001; Nagy et al., 2004; Bianco et al., 2007) and lattice spacing (Chung et al., 2011), with increased ionic strength (charge shielding) and increased lattice spacing (electrostatic force is inversely proportional to distance squared according to Coulomb's Law reducing the binding of PEVK and actin. In addition, S100A1, a calcium-binding protein found in the heart, inhibits the interaction between PEVK and actin in a calcium-dependent manner (Yamasaki et al., 2001), which suggests that PEVK-actin forces may depend on the physiological state of the heart. PEVK-actin interaction has been well-studied for the constitutive PEVK element that is the entire PEVK sequence in the N2B isoform, but evidence for interaction between the additional N2BA isoform PEVK exons and actin is lacking. However, differential binding occurs between actin
A
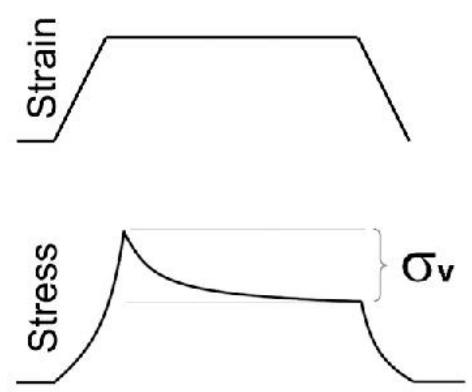

Time
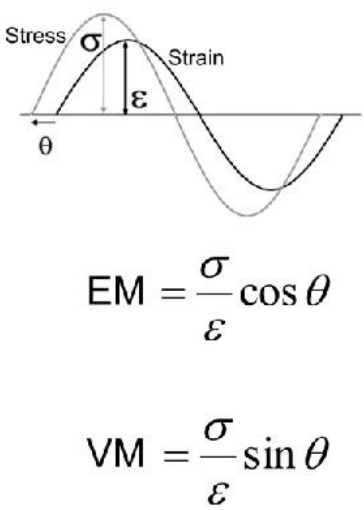

Fig. 5. A) The relationship between stress and strain in cardiac muscle. The difference between peak stress and steady-state stress is due to viscosity. B) Stress leads strain when sinusoidal length changes are imposed on myocardial tissue and is due to the presence of viscosity. The elastic modulus (EM) and viscous modulus (VM) are determined from the amplitudes of stress and strain and the phase difference between them. 
and PEVK segments of varying glutamate density (Nagy et al. 2004), which suggests that PEVK-actin interaction may differ between N2B and N2BA titin isoforms. Viscosity in the cardiac cell depends on the speed that intra-sarcomeric proteins slide past each other and is directly related to heart rate. Therefore, titin-based viscosity is predicted to be higher in small mammals (i.e. mice) with high heart rates compared to humans. However, the human heart rate can triple during exercise while rodent heart rates only slightly increase, which suggests that viscosity may be physiologically more relevant in larger mammals. It should be noted, however, that viscosity is greatest in cells and tissues following rest and that repeated stretches remove much of the viscous contribution (Helmes et al., 1999; Nedrud et al., 2011), although a significant steady-state level of viscosity remains. Repetitive stretches also reduced viscosity in isolated single titin molecules (Kellermayer et al., 2001).

\section{Titin and signaling pathways}

It is clear that titin isoform composition largely defines titinbased passive stiffness and that changes in hemodynamic load affect titin isoform expression, but what is less clear, and extremely interesting, is the pathways responsible for this adaptive dynamic. Significant contributions have recently been made towards a better understanding of titin's role in stretch-sensing and mechanical signaling. Although more work is needed to fully elucidate the details of titin's involvement, it has become clear that various regions along titin (Z-disk, I-band, and M-band) play a role in complex cell signaling pathways (Fig. 6).

\subsection{Z-Disk structure and signaling}

The Z-disk is a protein-dense region in the sarcomere that is responsible for maintaining sarcomere structure and mechanically connecting adjacent half-sarcomeres. The $\mathrm{N}$-terminus of titin contains two Ig domains (Z1/Z2) that embed titin in the Z-disk via strong contacts with proteins including T-cap (also called telethonin) and $\alpha$-actinin, the latter which crosslinks titin and the thin filament (Ohtsuka et al., 1997; Sorimachi et al., 1997). Also, the near
Z-disk portion of titin binds strongly to the thin filament and helps to separate titin's I-band region from interacting with the Z-disk (Granzier et al., 1997; Linke et al., 1997). Within the Z-disk, a single T-cap protein connects two titin molecules from the same halfsarcomere in an anti-parallel, sandwiched structure (Zou et al., 2006), which leads to strong $\beta$-strand crosslinking that likely functions to anchor titin at the Z-disk (Gregorio et al., 1998; Lee et al., 2006). A proposed mechanism for titin-based stretch sensing stems from T-cap's interaction with muscle LIM protein (MLP). MLP is an essential nuclear regulator of myogenic differentiation (Arber et al., 1994) and promotes myogenesis (Kong et al., 1997). Stretching cardiac myocytes in culture induces expression of brain natriuretic peptide (BNP) and atrial natriuretic factor (ANF), which are well-known stretch response markers, and this response was absent in MLP-null mice (Knoll et al., 2002); this suggests that MLP could be involved in stretch sensing through an interaction with T-cap and titin in the Z-disk. The coupling between sarcomere stress and Z-disk strain is shown by the fact that lattice spacing between thin filaments anchored in the Z-disk is altered by actomyosin-based active tension (Irving et al.,1998) and titin-based passive tension (Irving et al., 2011). In addition, T-cap mutations have been linked to hypertrophic cardiomyopathy (HCM) and dilated cardiomyopathy (DCM) with the disease phenotype hypothesized to arise from impaired binding between T-cap, titin, and other Z-disk proteins (Hayashi et al., 2004; Bos et al., 2006). MLP mutations that lead to mechanical stress signaling impairment have also been linked to HCM (Knoll et al., 2002; Geier et al., 2008), and MLP KO mice exhibit enlarged failing hearts (Arber et al., 1997).

Various other binding partners of titin's Z-disk region imply that titin's presence in the Z-disk is structurally very important. Obscurin, another giant sarcomeric protein, interacts with the $\mathrm{N}$ terminus of titin via two Ig-like domains in obscurin (Young et al., 2001) and plays an important role in Z-disk formation (Bowman et al., 2008). Titin's Z-disk region also interacts with filamin (Labeit et al., 2006), which links titin to integrin and the focal adhesion complex (Samarel, 2005; Romer et al., 2006) and suggests that titin behavior may be a stimulus that affects other cellular processes. To summarize, the protein-rich Z-disk of the sarcomere involves complex interplay between various proteins, including the

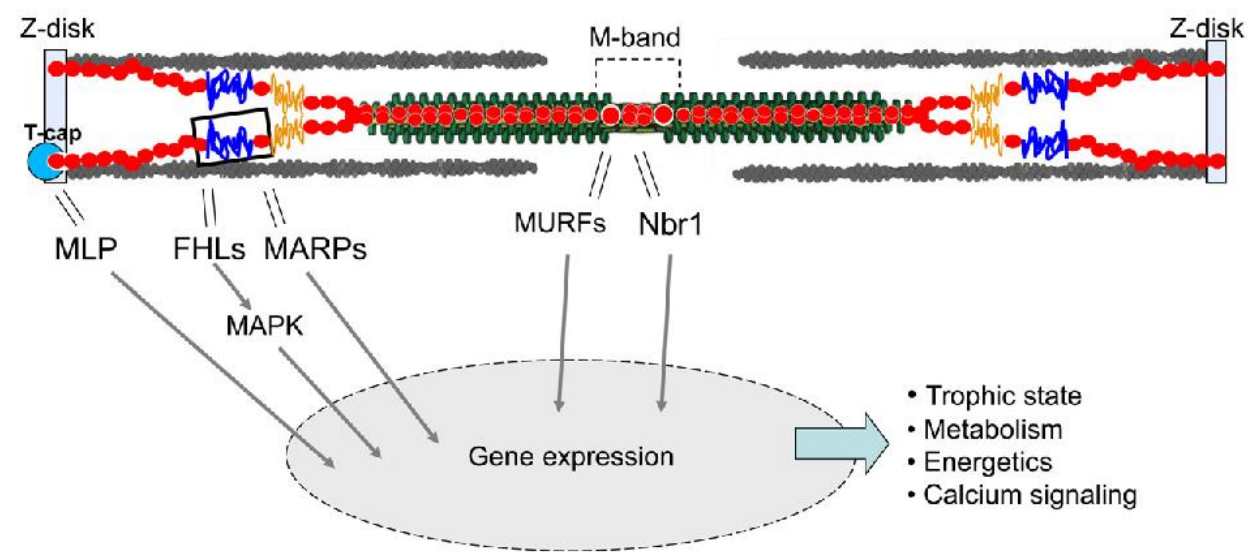

Fig. 6. Signaling hotspots in titin. At the Z-disk, the titin-binding protein T-cap interacts with muscle LIM protein (MLP), a nuclear regulator of myogenesis. In the I-band, the N2B and N2A elements interact with four-and-a-half-LIM protein (FHL) and muscle ankyrin-repeat proteins (MARPs), respectively. The N2B element and FHL are thought to form a forcedependent stretch-sensing complex that also involves components of MAPK signaling, which regulates hypertrophy. The interaction of the N2A element and MARPs is hypothesized to influence transcription as a function of mechanical strain. The M-band region of titin interacts with MURFs and Nbr1, and these interactions may play an important role in calcium signaling and hypertrophy. 
$\mathrm{N}$-terminus of titin. Disruption of this network is present in numerous cardiomyopathies and likely disrupts the role of titin in mechanotransduction.

\subsection{I-band signaling}

The signaling hotspots within the I-band region of cardiac titin are the N2B and N2A elements. The cardiac-specific N2B element interacts with two members of the four-and-a-half-LIM domain protein family, FHL1 (Sheikh et al., 2008) and FHL2 (Lange et al., 2002), that can localize in the nucleus to act as transcription coactivators (Scholl et al., 2000). FHL1 is suggested to interact with the N2B element to form a stretch-sensing complex that is downstream of G-protein-coupled receptor (GPCR) signaling (Sheikh et al., 2008). This complex also involves Raf1, MEK2, and ERK2, which are components of the MAPK signaling pathway; the MAPK pathway is highly-involved in hypertrophy signaling (Muslin, 2008). FHL1 KO mice displayed an attenuated hypertrophic response and a beneficial functional response to transverse aortic constriction (TAC)-induced pressure overload that leads to hypertrophy in control mice (Sheikh et al., 2008). This suggests that the $\mathrm{N} 2 \mathrm{~B} / \mathrm{FHL} 1$ complex is a stretch sensor in cardiomyocytes. FHL2 has numerous binding partners that play a wide-ranging functional role in the cell (Johannessen et al., 2006) and may play a role in stretch-induced hypertrophy signaling through interaction with the N2B element (Granzier et al., 2009). In a mouse model where exon 49 was deleted (N2B KO; deletion of entire N2B element) FHL2 levels were significantly down-regulated and the hearts were atrophied (Radke et al., 2007). However, when the full PEVK element of the N2B titin isoform was removed (PEVK KO), which necessarily increases the strain placed on the N2B element, FHL2 (as well as FHL1) was up-regulated and cardiac hypertrophy was present (Granzier et al., 2009). It was hypothesized that strain on the N2B spring element induces structural changes that favor FH binding and the assembly of a signaling complex that induces hypertrophy, and that elimination of this complex accounts for the atrophy response seen in the N2B KO model. The signaling network that connects this proposed mechanosensor to the trophic response requires further investigation.

The N2A element, which is found in the N2BA titin isoform but not the N2B isoform, is also involved in signaling pathways. The unique sequence between Ig80 and Ig81 within the N2A element binds to cardiac ankyrin-repeat protein (CARP), diabetes-related ankyrin-repeat protein (DARP), and ankyrin-repeat domainprotein-2 (Ankrd2) (Miller et al., 2003), all of which belong to the muscle ankyrin-repeat protein family (MARPs). MARPs are thought to relocate from the I-band to the nucleus following mechanical stress to regulate transcription (Kojic et al., 2004). It has been proposed that the N2A element forms a stretch sensing complex that includes MARPs, myopalladin, and the calpain protease p94. In cultured cardiomyocytes from rat, external stretch induced differential localization of CARP and DARP, including increased DARP staining at the intercalated disks (Miller et al., 2003), which are protein-dense structures that mechanically-link neighboring cells and interact with titin at terminal sarcomeres (Bennett et al., 2006).

\subsection{M-Band signaling}

The M-band region of titin, which binds to the thick filamen near the middle of the sarcomere, is also involved in signaling pathways. Although the thick filament bears force in the sarcomere, it is only mildly compliant (Irving et al., 2011). Titin domains A168 A170 (comprised of two Ig-like domains and one fibronectin type 3 domain; Fig. 3), which are located immediately N-terminal to titin's kinase domain, bind to MURF-1 (McElhinny et al., 2002; Mrosek et al., 2007), an E3 ubiquitin ligase that targets muscle proteins for proteasomal degradation. MURF-1 also binds to glucocorticoid modulatory element binding protein-1 (GMEB-1) (McElhinny et al., 2002), a transcriptional regulator. This titin-MURF interaction is thought to regulate myofibril turnover and the trophic state of striated muscle, although ubiquitination levels of titin were unchanged in a MURF-1 KO mouse (Witt et al., 2005). However, MURF-1 and MURF-2 double KO mice developed extreme cardiac hypertrophy (Witt et al., 2008), which suggests that MURFs are important for regulating myogenesis in the heart.

The titin kinase (TK) domain is adjacent to the MURF binding site and has been thoroughly researched because, as titin's only catalytic domain, it has the potential to act as a direct biological force sensor. In-depth study has also been aroused by the determination of TK's crystal structure (Mayans et al., 1998). Although it is suggested that TK phosphorylates T-cap (Mayans et al., 1998), supporting data is lacking. This is likely due to TK's dual autoinhibitory mechanism: for the catalytic site of TK to be solventaccessible, the C-terminal tail needs to be removed from its native state and tyrosine-170 needs to be phosphorylated (Mayans et al., 1998). AFM experiments (Puchner et al., 2008) and molecular dynamics simulations (Grater et al., 2005) have determined that external force acting on the termini of TK removes the regulatory $\mathrm{C}$ terminal tail from blocking the ATP-binding site, which suggests that TK activity may be force activated; however this has not been shown directly. Two-hybrid assays and precipitation experiments with various truncated TK constructs showed that absence of the regulatory tail was necessary for interaction with Nbr1 (Lange et al., 2005), a zinc-finger protein. Such proteins have been found to act as scaffolds for signalosome assembly (Pawson and Scott, 1997), and it was also found that p62, another zinc-finger protein, bound to Nbr1; in vitro assays also found that Nbr1 and p62 (to a much lower level) are TK substrates (Lange et al., 2005). MURF-2, which is involved in myofibrillogenesis (Spencer et al., 2000), was also dentified as a ligand of p62. It was suggested that Nbr1 acts as a scaffolding protein that assembles sarcomeric signalosomes that include p62, MURF-2, and titin kinase (Lange et al., 2005). In neonatal mice, deletion of TK results in cardiomyopathy and early death (Gotthardt et al., 2003), and a MerCreMer transgenic model, which allowed removal of TK in adult mice, exhibited an attenuated response to adrenergic stimulation and extracellular calcium, severe cardiac hypertrophy, and congestive heart failure (Peng et al., 2007). This collection of data suggests that titin kinase plays a force-dependent role in structural development and hypertrophy signaling.

\section{Future directions}

Due to its immense size and multi-functional role, there is still much to be learned about titin's contribution in cardiac health and disease. The relationship between titin isoform composition and passive tension is well-understood, but the splicing pathways that respond to cardiac insult and bias expression of titin isoforms are largely unknown. Elucidation of these splice pathways will unlock the possibility of using pharmacological therapies to modulate titin isoform expression in chronic pathologies. In addition, the physics of titin-ligand interactions needs to be better understood, in particular the force-dependence of these interactions. It is likely that sarcomere stress influences titin-ligand binding differently along the titin molecule; for instance, the I-band region of titin may be more suited to sense strain than the Z-disk and M-band regions of titin because it is not strongly bound to other sarcomeric filaments, and integrative experiments will be required to determine the physical catalysts and physiological responses of titin-ligand interactions involved in signaling pathways. Although great 
progress has been made in the study of titin, more work is needed to shed light on titin biomechanics. Fortunately, various cuttingedge techniques, including single molecule force spectroscopy and generation of novel mouse models, are being successfully applied to titin research, and it seems to be only a matter of time before the mechanisms that link titin strain and the cellular response are critically understood.

\section{Editors' note}

Please see also related communications in this issue by Werdich et al. (2012) and Fabritz et al. (2012).

\section{Acknowledgments}

This review was supported by NIH training grant GM084905 and an award from the American Heart Association 11PRE7370083 to B.A and by NIH HL062881 to H.G.

\section{References}

Anderson, B., Bogomolovas, J., et al., in press.

Anderson, B.R., Bogomolovas, J., et al., 2010. The effects of PKCalpha phosphorylation on the extensibility of titin's PEVK element. J. Struct. Biol. 170 (2), 270-277. Arber, S., Halder, G., et al., 1994. Muscle UM protein, a novel essential regulator of myogenesis, promotes myogenic differentiation. Cell 79 (2), 221-231.

Arber, S., Hunter, J.J., et al., 1997. MLP-deficient mice exhibit a disruption of cardiac cytoarchitectural organization, dilated cardiomyopathy, and heart failure. Cell 88 (3), 393-403.

Bang, M.L., Centner, T., et al., 2001. The complete gene sequence of titin, expression of an unusual approximately $700-\mathrm{kDa}$ titin isoform, and its interaction with obscurin identify a novel Z-line to I-band linking system. Circ. Res. 89 (11).

1065-1072.
Belin, R.J., Sumandea, M.P., et al., 2007. Augmented protein kinase C-alpha-induced myofilament protein phosphorylation contributes to myofilament dysfunction in experimental congestive heart failure. Circ. Res. 101 (2), 195-204.

Bell, G.L., 1978. Models for the specific adhesion of cells to cells. Science 200 (4342), $618-627$.

Bell, S.P., Nyland, L., et al., 2000. Alterations in the determinants of diastolic suction during pacing tachycardia. Circ. Res. 87 (3), 235-240.

Bennett, P.M., Maggs, A.M., et al., 2006. The transitional junction: a new functional subcellular domain at the intercalated disc, Mol, Biol. Cell 17 (4), 2091-2100.

Bianco, P., Nagy, A., et al., 2007. Interaction forces between F-Actin and titin PEVK domain measured with optical tweezers. Biophys. J. 93 (6), 2102-2109.

Binnig, G., Quate, C.F., et al., 1986. Atomic force microscope. Phys. Rev. Lett. 56 (9), 930-933.

Bos, J.M., Poley, R.N., et al., 2006. Genotype-phenotype relationships involving hypertrophic cardiomyopathy-associated mutations in titin, muscle UM protein, and telethonin. Mol. Genet. Metab. 88 (1), 78-85.

Bowman, A.L, Catino, D.H., et al., 2008. The Rho-guanine nucleotide exchange factor domain of obscurin regulates assembly of titin at the Z-disk throug interactions with ran binding protein 9. Mol. Biol. Cell 19 (9), 3782-3792.

Cazorla, O., Freiburg, A., et al., 2000. Differential expression of cardiac titin isoforms and modulation of cellular stiffness. Circ. Res. 86 (1), 59-67.

Cheng, S.M., Cetinkaya, M., et al., 2010. How sequence determines elasticity of disordered proteins. Biophys. J. 99 (12), 3863-3869.

Chung, C.S., Granzier, H.L., 2011. Contribution of titin and extracellular matrix to passive pressure and measurement of sarco

Chung, C.S., Methawasin, M., et al., 2011. Titin based viscosity in ventricular physiology: an integrative investigation of PEVK-actin interactions. J. Mol. Cell Cardiol. 51 (3), 428-434

Cicogna, A.C., Robinson, K.G., et al., 1999. Direct effects of colchicine on myocardial function: studies in hypertrophied and failing spontaneously hypertensive rats. Hypertension 33 (1), 60-65.

Conrad, C.H., Brooks, W.W., et al., 1995. Myocardial fibrosis and stiffness with hypertrophy and heart failure in the spontaneously hypertensive rat. Circulation 91 (1), 161-170.

de Tombe, P.P., ter Keurs, H.E., 1992. An internal viscous element limits unloaded velocity of sarcomere shortening in rat myocardium. J. Physiol. 454, 619-642.

Dudko, O.K., Hummer, G., et al., 2008. Theory, analysis, and interpretation of singlemolecule force spectroscopy experiments. Proc. Natl. Acad. Sci. USA 105 (41), 15755-15760.

Fabritz, L., Fortmüller, L., Yu, T.Y., Paul, M., Kirchhof, P., 2012. Can preload-reducing therapy prevent disease progression in arrhythmogenic right ventricular cardiomyopathy? Experimental evidence and design of a clinical trial. Prog. Biophys. Mol. Biol. $110(2-3), 340-346$.
Fowler, S.B., Best, R.B., et al., 2002. Mechanical unfolding of a titin Ig domain: structure of unfolding intermediate revealed by combining AFM, molecular
dynamics simulations, NMR and protein engineering. J. Mol. Biol. 322 (4), $841-849$.

Freiburg, A., Trombitas, K., et al., 2000. Series of exon-skipping events in the elastic spring region of titin as the structural basis for myofibrillar elastic diversity. Circ. Res. 86 (11), 1114-1121.

Fukuda, N., Wu, Y., et al., 2005. Phosphorylation of titin modulates passive stiffness of cardiac muscle in a titin isoform-dependent manner. J. Gen. Physiol. 125 (3) 257-271.

Fukushima, H., Chung, C.S., et al., 2010. Titin-isoform dependence of titin-actin interaction and its regulation by $\$ 100 \mathrm{~A} 1 / \mathrm{Ca}^{2+}$ in skinned myocardium. J. Biomed. Biotechnol. 2010, 727239.

Garcia-Manyes, S., Brujic, J., et al., 2007. Force-clamp spectroscopy of single-protein monomers reveals the individual unfolding and folding pathways of 127 and ubiquitin. Biophys. J. 93 (7), 2436-2446.

Geier, C., Gehmlich, K., et al., 2008. Beyond the sarcomere: CSRP3 mutations cause

hypertrophic cardiomyopathy. Hum. Mol. Genet. 17 (18), $2753-2765$.
Gotthardt, M., Hammer, R.E., et al., 2003. Conditional expression of mutant M-line titins $278(8), 6059-6065$.

Granzier, H., Kellermayer, M., et al., 1997. Titin elasticity and mechanism of passive force development in rat cardiac myocytes probed by thin-filament extraction. Biophys. J. 73 (4), 2043-2053.

Granzier, H.L., Labeit, S., 2005. Titin and its associated proteins: the third myofilament system of the sarcomere. Adv. Protein Chem. 71, 89-119.

Granzier, H.L, Radke, M.H., et al., 2009. Truncation of titin's elastic PEVK region . leads to cardiomyopathy with diastolic dysfunction. Circ. Res. 105 (6), 557-564 er, F., Shen, J., et al., 2005. Mechanically induced titin kinase activation studied

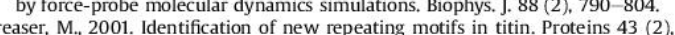
$145-149$.

Greaser, M.L., Krzesinski, P.R., et al., 2005. Developmental changes in rat cardiac titin/connectin: transitions in normal animals and in mutants with a delayed
(a) titin/connectin: transitions in normal animals and in mutants with a delayed

Gregorio, C.C., Trombitas, K., et al., 1998. The NH2 terminus of titin spans the Z-disc: its interaction with a novel 19-kD ligand (T-cap) is required for sarcomeric
(a) integrity. J. Cell Biol. $143(4), 1013-1027$.

Gutierrez-Cruz, G., Van Heerden, A.H., et al., 2001. Modular motif, structural folds and affinity profiles of the PEVK segment of human fetal skeletal muscle titin. J. Biol. Chem. 276 (10), 7442-7449.

Hayashi, T., Arimura, T., et al., 2004. Tcap gene mutations in hypertrophic cardiomyopathy and dilated cardiomyopathy. J. Am. Coll. Cardiol. 44 (11) 2192-2201.

Helmes, M., Trombitas, K., et al., 1999. Mechanically driven contour-length adjustment in rat cardiac titin's unique N2B sequence: titin is an adjustable spring. Circ. Res. 84 (11), 1339

Hidalgo, C., Hudson, B., et al., 2009. PKC phosphorylation of titin's PEVK element: a novel and conserved pathway for modulating myocardial stiffness. Circ. Res. 105 (7), 631-638, 617 pp. following 638.

Hudson, B.D., Hidalgo, C.G., et al., 2010. Excision of titin's cardiac PEVK spring element abolishes PKCalpha-induced increases in myocardial stiffness. J. Mol. Cell Cardiol. 48 (5), 972-978.

Hummer, G., Szabo, A., 2003. Kinetics from nonequilibrium single-molecule pulling experiments. Biophys. J. 85 (1), 5-15.

Improta, S., Politou, A.S., et al., 1996. Immunoglobulin-like modules from titin Iband: extensible components of muscle elasticity. Structure 4 (3), 323-337.

Irving, T., Li, Q., et al., 1998. Z/I and A-band lattice spacings in frog skeletal muscle: effects of contraction and osmolarity. J. Muscle Res. Cell Motil. 19 (7), 811-823.

Irving, T., Wu, Y., et al., 2011. Thick-filament strain and interfilament spacing in passive muscle: effect of titin-based passive tension. Biophys. J. 100 (6), 1499-1508

Johannessen, M., Moller, S., et al., 2006. The multifunctional roles of the four-and-ahalf-LIM only protein FHL2. Cell. Mol. Life Sci. 63 (3), 268-284.

Kabsch, W., Mannherz, H.G., et al., 1990. Atomic structure of the actin:DNase complex. Nature 347 (6288), 37-44.

Kellermayer, M.S., Smith, S.B., et al., 1997. Folding-unfolding transitions in single titin molecules characterized with laser tweezers. Science 276 (5315), 1112-1116.

Kellermayer, M.S., Smith, S.B., et al., 2001. Mechanical fatigue in repetitively stretched single molecules of titin. Biophys. J. 80 (2), 852-863.

Knoll, R., Hoshijima, M., et al., 2002. The cardiac mechanical stretch sensor machinery involves a $\mathrm{Z}$ disc complex that is defective in a subset of human dilated cardiomyopathy. Cell 111 (7), 943-955.

Kojic, S., Medeot, E., et al., 2004. "The Ankrd2 protein, a link between the sarcomere and the nucleus in skeletal muscle. J. Mol. Biol, 339 (2), 313-325.

Kong, Y., Flick, M.J., et al., 1997. Muscle LIM protein promotes myogenesis by enhancing the activity of MyoD. Mol. Cell. Biol. 17 (8), 4750-4760.

Kruger, M., Linke, W.A., 2006. Protein kinase-A phosphorylates titin in human heart muscle and reduces myofibrillar passive tension. J. Muscle Res. Cell Motil. 27 (5-7), 435-444.

Kruger, M., Kotter, S., et al., 2009. Protein kinase G modulates human myocardia passive stiffness by phosphorylation of the titin springs. Circ. Res. 104 (1), 87-94 Labeit, S. Kolmerer, B. 1995. Titins: giant proteins in charge of muscle ultrastructure and elasticity. Science 270 (5234), 293-296. 
Labeit, S., Lahmers, S., et al., 2006. Expression of distinct classes of titin isoforms in striated and smooth muscles by alternative splicing, and their conserved interaction with filamins. J. Mol. Biol, 362 (4), 664-68

Lahmers, S., Wu, Y., et al., 2004. Developmental control of titin isoform expression and passive stiffness in fetal and neonatal myocardium. Circ. Res. 94 (4), 505-513.

Lange, S., Auerbach, D., et al., 2002. Subcellular targeting of metabolic enzymes to titin in heart muscle may be mediated by DRAL/FHL-2. J. Cell Sci. 115 (Pt 24), 4925-4936.

Lange, S., Xiang, F., et al., 2005. The kinase domain of titin controls muscle gene expression and protein turnover. Science 308 (5728), 1599-1603.

Leake, M.C., Grutzner, A., et al., 2006. Mechanical properties of cardiac titin's N2Bregion by single-molecule atomic force spectroscopy. J. Struct. Biol. 155 (2), 263-272.

Lee, E.H., Gao, M., et al., 2006. Mechanical strength of the titin Z1Z2-telethonin complex. Structure 14 (3), 497-509.

Lee, E.H., Hsin, J., et al., 2010. Tertiary and secondary structure elasticity of a six-Ig titin chain. Biophys. J. 98 (6), 1085-1095.

Li, H., Carrion-Vazquez, M., et al., 2000. Point mutations alter the mechanical stability of immunoglobulin modules. Nat. Struct. Biol. $7(12), 1117-1120$.

Li, H., Oberhauser, A.F., et al., 2001. Multiple conformations of PEVK proteins detected by single-molecule techniques. Proc. Natl. Acad. Sci. USA 98 (19), 10682-10686.

Li, H., Linke, W.A., et al., 2002. Reverse engineering of the giant muscle protein titin. Nature 418 (6901), 998-1002.

Linke, W.A., Ivemeyer, M., et al., 1997. Actin-titin interaction in cardiac myofibrils: probing a physiological role. Biophys. J. 73 (2), 905-919.

linke, W.A., Kulke, M., et al., 2002. PEVK domain of titin: an entropic spring with actin-binding properties. J. Struct. Biol. 137 (1-2), 194-205.

Linke, W.A., Grutzner, A., 2008. Pulling single molecules of titin by AFM - recent advances and physiological implications. Pflug. Arch. Eur.J. Phy. 456 (1), 101-115.

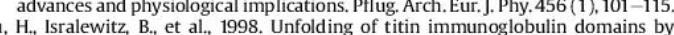
steered molecular dynamics simulation. Biophys. J. 75 (2), 662-671.

Ma, K. Kan, L. et al, 2001. Polyproline II helix is a key structural motif of the elastic PEVK segment of titin. Biochemistry 40 (12), 3427-3438.

PEVK segment of titin. Biochemistry 40 (12), 3427-3438. arino, M., Svergun, D.I., et al., 2005. Poly-lg tandems from I-band titin share extended domain arrangements irrespective of the distinct features

Marko, J.F., Siggia, E.D., 1995. Stretching DNA. Macromolecules 28, 8759-8770.

Marko, J.F., Siggia, E.D., 1995. Stretching DNA. Macromolecules 28, 8759-8770. modules. Nature 402 (6757), $100-103$

modules. Nature 402 (6757), $100-103$.
Mayans, O., van der Ven, P.F., et al., 1998 . Structural basis for activation of the titin kinase domain during myofibrillogenesis. Nature 395 (6705), 863-869.
kins.

kinase domain during myofibrillogenesis. Nature 395 (6705), 863-869.
. reversible disulphide bridge formation in the elasticity of the muscle protein
res reversible disulphide bridge fo

McElhinny, A.S., Kakinuma, K., et al., 2002. Muscle-specific RING finger-1 interacts with titin to regulate sarcomeric M-line and thick filament structure and may have nuclear functions via its interaction with glucocorticoid modulatory element binding protein-1.J. Cell Biol. 157 (1), 125-136

Miller, M.K., Bang, M.L., et al., 2003. The muscle ankyrin repeat proteins: CARP, ankrd2/Arpp and DARP as a family of titin filament-based stress response molecules. J. Mol. Biol. 333 (5), 951-964.

Molkentin, J.D., Dorn, G.W., 2001. Cytoplasmic signaling pathways that regulate cardiac hypertrophy. Annu. Rev. Physiol. 63, 391-426.

Mrosek, M., Labeit, D., et al., 2007. Molecular determinants for the recruitment of the ubiquitin-ligase MuRF-1 onto M-line titin. Faseb J. 21 (7), 1383-1392.

Muslin, A.J., 2008. MAPK signalling in cardiovascular health and disease: molecular mechanisms and therapeutic targets. Clin. Sci. $115(7-8), 203-218$

Nagueh, S.F., Shah, G., et al., 2004. Altered titin expression, myocardial stiffness, and left ventricular function in patients with dilated cardiomyopathy. Circulation $110(2), 155-162$

Nagy, A., Cacciafesta, P., et al., 2004. Differential actin binding along the PEVI domain of skeletal muscle titin. J. Cell Sci. 117 (Pt 24), 5781-5789.

Neagoe, C., Kulke, M., et al., 2002. Titin isoform switch in ischemic human heart disease. Circulation $106(11), 1333-1341$

Neagoe, C., Opitz, C.A., et al., 2003. Gigantic variety: expression patterns of titin isoforms in striated muscles and consequences for myofibrillar passive stiffness. J. Muscle Res. Cell Motil. 24 (2-3), 175-189.

Nedrud, J., Labeit, S., et al., 2011. Mechanics on myocardium deficient in the N2B region of titin: the cardiac-unique spring element improves efficiency of the cardiac cycle. Biophys. J. 101 (6), 1385-1392.

Oberhauser, A.F., Hansma, P.K., et al., 2001. Stepwise unfolding of titin under forceclamp atomic force microscopy. Proc. Natl. Acad. Sci. USA 98 (2), 468-472.

Ohtsuka, H., Yajima, H., et al., 1997. Binding of the N-terminal 63 kDa portion of connectin/titin to alpha-actinin as revealed by the yeast two-hybrid system. Febs. Lett. 401 (1), 65-67.

Opitz, C.A., Leake, M.C., et al., 2004. Developmentally regulated switching of titin size alters myofibrillar stiffness in the perinatal heart. Circ. Res. 94 (7), 967-975.

Pawson, T., Scott, J.D., 1997. Signaling through scaffold, anchoring, and adapto proteins. Science 278 (5346), 2075-2080.

Peng. J., Raddatz, K., et al., 2007. Cardiac hypertrophy and reduced contractility in hearts deficient in the titin kinase region. Circulation 115 (6), 743-751.
Pfuhl, M., Pastore, A., 1995. Tertiary structure of an immunoglobulin-like domain from the giant muscle protein titin: a new member of the I set. Structure 3 (4). 391-401.

Porter, K.E., Turner, N.A., 2009. Cardiac fibroblasts: at the heart of myocardial remodel ing. Pharmacol. Therapeut. 123 (2), 255-278.

Puchner, E.M., Alexandrovich, A., et al., 2008. Mechanoenzymatics of titin kinase. Proc, Natl. Acad. Sci. USA 105 (36), 13385-13390.

Radke, M.H., Peng, J., et al., 2007. Targeted deletion of titin N2B region leads to diastolic dysfunction and cardiac atrophy. Proc. Natl. Acad. Sci. USA $104(9)$ 3444-3449.

Rief, M., Gautel, M., et al., 1997. Reversible unfolding of individual titin immunoglobulin domains by AFM. Science 276 (5315), 1109-1112.

Romer, L.H., Birukov, K.G., et al., 2006. Focal adhesions: paradigm for a signaling nexus. Circ. Res. 98 (5), 606-616.

Samarel, A.M., 2005. Costameres, focal adhesions, and cardiomyocyte mechanotransduction. Am. J. Physiol. Heart Circ. Physiol. 289 (6), H2291-H2301.

Scholl, F.A., McLoughlin, P., et al., 2000. DRAL is a p53-responsive gene whose fou and a half $\mathbf{U M}$ domain protein product induces apoptosis. J. Cell Biol. 151 (3), 495-505.

Sheikh, F., Raskin, A., et al., 2008. An FHL1-containing complex within the cardiomyocyte sarcomere mediates hypertrophic biomechanical stress responses in mice. J. Clin. Invest. 118 (12), 3870-3880.

Sorimachi, H., Freiburg, A., et al., 1997. Tissue-specific expression and al pha-actinin binding properties of the Z-disc titin: implications for the nature of vertebrate binding properties of the Z-disc titin:

Spencer, J.A., Eliazer, S., et al., 2000. Regulation of microtubule dynamics and myogenic differentiation by MURF, a striated muscle RING-finger protein.J. Cell Biol. 150 (4), 771-784.

Stacklies, W., Vega, M.C., et al., 2009. Mechanical network in titin immunoglobulin from force distribution analysis. PLoS Comput. Biol. 5 (3), e1000306

Taylor, M., Graw, S., et al., 2011. Genetic variation in titin in arrhythmogenic right ventricular cardiomyopathy-overlap syndromes. Circulation 124 (8) $876-885$

Trombitas, K., Wu, Y., et al., 2001. Cardiac titin isoforms are coexpressed in the halfsarcomere and extend independently. Am. J. Physiol. Heart Circ. Physiol. 281 (4) H1793-H1799.

van Heerebeek, L., Borbely, A., et al., 2006. Myocardial structure and function differ in systolic and diastolic heart failure. Circulation 113 (16), 1966-1973.

von Castelmur, E., Marino, M., et al., 2008. A regular pattern of Ig super-motifs defines segmental flexibility as the elastic mechanism of the titin chain. Proc. Natl. Acad. Sci. USA 105 (4), 1186-1191.

Wang $K$, Mccarter, R et al. 1991. Regulation of skeletal-muscle stiffness and elasticity by titin isoforms - a test of the segmental extension model of resting tension. Proc. Natl. Acad. Sci. USA 88 (16), 7101-7105.

Warren, C.M., Jordan, M.C., et al., 2003. Titin isoform expression in normal and hypertensive myocardium. Cardiovasc. Res. 59 (1), 86-94.

Watanabe, K., Muhle-Goll, C., et al., 2002a. Different molecular mechanics displayed by titin's constitutively and differentially expressed tandem Ig segments. by titin's constitutively and differ. $137(1-2), 248-258$.

Watanabe, K., Nair, P., et al., 2002b. Molecular mechanics of cardiac titin's PEVK and N2B spring elements. J. Biol. Chem. 277 (13), 11549-11558.

Weber, K.T., 1989. Cardiac interstitium in health and disease: the fibrillar collagen network. J. Am. Coll. Cardiol. 13 (7), 1637-1652.

Werdich, A.A., Brzezinski, A., Jeyaraj, D., Sabeh, M.K., Ficker, E., Wan, X MacRae, C.A., Rosenbaum, D.S., 2012. The zebrafish as a novel animal model to study the molecular mechanisms of mechano-electrical feedback in the heart Prog. Biophys. Mol. Biol. 110 (2-3), 154-165.

Witt, S.H., Granzier, H., et al., 2005. MURF-1 and MURF-2 target a specific subset of myofibrillar proteins redundantly: towards understanding MURF-dependen muscle ubiquitination. J. Mol. Biol. 350 (4), 713-722.

Witt, C.C., Witt, S.H., et al., 2008. Cooperative control of striated muscle mass and metabolism by MuRF1 and MuRF2. Embo. J. 27 (2), 350-360.

Wu, Y., Cazorla, O., et al., 2000. Changes in titin and collagen underlie diastoli stiffness diversity of cardiac muscle. J. Mol. Cell Cardiol. 32 (12), 2151 -2162.

$\mathrm{Wu}, \mathrm{Y} .$, Bell, S.P., et al., 2002. Changes in titin isoform expression in pacing-induced cardiac failure give rise to increased passive muscle stiffness. Circulation 106 (11), 1384-1389,

Yamasaki, R., Berri, M., et al., 2001. Titin-actin interaction in mouse myocardium: passive tension modulation and its regulation by calcium/S100A1. Biophys. J. 8 (4), 2297-2313.

Yamasaki, R., Wu, Y., et al., 2002. Protein kinase A phosphorylates titin's cardiacspecific N2B domain and reduces passive tension in rat cardiac myocytes Circ. Res. 90 (11), 1181-1188.

Young, P., Ehler, E., et al., 2001. Obscurin, a giant sarcomeric Rho guanine nucleotide exchange factor protein involved in sarcomere assembly. J. Cell Biol. 154 (1). $123-136$

Zhmurov, A., Dima, R.I., et al., 2010. Order statistics theory of unfolding of multimeric proteins. Biophys. J. 99 (6), 1959-1968.

Zhu, Y., Bogomolovas, J., et al., 2009. Single molecule force spectroscopy of the cardiac titin N2B element: effects of the molecular chaperone alphaB-crystallin with disease-causing mutations. J. Biol. Chem. 284 (20), 13914-13923.

Zou, P., Pinotsis, N., et al., 2006. Palindromic assembly of the giant muscle protein titin in the sarcomeric Z-disk. Nature 439 (7073), 229-233. 
Appendix C

Anderson, B.R., et al., The effects of PKCalpha phosphorylation on the extensibility of titin's PEVK element. J Struct Biol, 2010. 170(2): p. 270-7. 


\title{
The effects of PKC $\alpha$ phosphorylation on the extensibility of titin's PEVK element
}

\author{
Brian R. Anderson ${ }^{\mathrm{a}, \mathrm{c}}$, Julius Bogomolovas ${ }^{\mathrm{b}}$, Siegfried Labeit ${ }^{\mathrm{b}}$, Henk Granzier ${ }^{\mathrm{c}, *}$ \\ ${ }^{a}$ Dept. of Physics, University of Arizona, Tucson, AZ, USA \\ ${ }^{b}$ Institute for Integrative Pathophysiology, Universitätsmedizin Mannheim, Germany \\ ${ }^{c}$ Dept. of Physiology and Sarver Molecular Cardiovascular Research Program, University of Arizona, Tucson, AZ, USA
}

\section{A R T I C L E I N F O}

\section{Article history:}

Received 30 November 2009

Received in revised form 1 February 2010

Accepted 3 February 2010

Available online 10 February 2010

\section{Keywords:}

Passive tension

Single molecule mechanics

Post-translational modifications

\begin{abstract}
A B S T R A C T
Post-translational modifications, along with isoform splicing, of titin determine the passive tension development of stretched sarcomeres. It was recently shown that PKC $\alpha$ phosphorylates two highly-conserved residues (S26 and S170) of the PEVK region in cardiac titin, resulting in passive tension increase. To determine how each phosphorylated residue affects myocardial stiffness, we generated three recombinant mutant PEVK fragments (S26A, S170A and S170A/S26A), each flanked by Ig domains. Single-molecule force spectroscopy shows that PKC $\alpha$ decreases the PEVK persistence length (from 0.99 to $0.68 \mathrm{~nm}$ ): the majority of this decrease is attributable to phosphorylation of S26. Before PKC $\alpha$, all three mutant PEVK fragments showed at least $40 \%$ decrease in persistence length compared to wildtype. Furthermore, Ig domain unfolding force measurements indicate that PEVK's flanking Ig domains are relatively unstable compared to other titin Ig domains. We conclude that phosphorylation of S26 is the primary mechanism through which PKC $\alpha$ modulates cardiac stiffness.
\end{abstract}

(c) 2010 Elsevier Inc. All rights reserved.

\section{Introduction}

Passive tension development in the sarcomere plays a crucia role in proper diastolic function. The giant protein titin, also called connectin, spans the half-sarcomere from Z-disk to M-line (Furst et al., 1988), and is responsible for the development of passive tension within the sarcomere (Horowits et al., 1986). Titin contains an extensible I-band region that comprises three distinct regions: serially-linked immunoglobulin (Ig)-like domains, the N2B element, and the PEVK region, which primarily contains proline (P), glutamate (E), valine (V), and lysine (K) residues (Labeit and Kolmerer, 1995). These three spring-like elements develop force when extended and, along with collagen, determine myocardial passive stiffness.

Isoform splicing is a long-term pathway that alters myocardial passive stiffness. Cardiac titin is composed of two isoforms: the small $(2.97 \mathrm{kDa}) \mathrm{N} 2 \mathrm{~B}$ titin and larger N2BA titin isoform (3.23.4 MDa). Exon splicing pathways determine the differential expression of titin's I-band element, and result in N2BA titin containing a larger PEVK domain than the N2B element, in addition to a variable number of Ig domains (Granzier and Labeit, 2005). The 188-residue PEVK sequence examined in this investigation is

* Corresponding author. Address: Dept. of Physiology and Sarver Molecular Cardiovascular Research Program, University of Arizona, P.O. Box 245217, Tucson, AZ 85724, USA. Fax: +15206267600

E-mail addresses: granzier@email.arizona.edu, henkgranzier@gmail.com ( $\mathrm{H}$. Granzier).

1047-8477/\$ - see front matter @ 2010 Elsevier Inc. All rights reserved.

doi:10.1016/j.jsb.2010.02.002 the entire PEVK domain of the cardiac N2B isoform, the main isoform in the left ventricle, but it is also present in the N2BA isoform (Freiburg et al., 2000). At a given extension, the cardiac N2B isoform develops more force than the N2BA isoform because of its shorter end-to-end length. This shorter isoform length results in a greater fractional extension of the elastic elements in the I-band, which leads to greater force development (Trombitas et al., 2000).

The critical importance of relative fractional extension in determining passive tension dynamics of the l-band is evident in spring element KO studies. Increased passive tension was measured in PEVK-KO mice and was attributed to the extensional increase in the spring-like N2B element (Granzier et al., 2009). Increase in passive tension was also measured in N2B-KO mice, and it was determined that the PEVK region extended most in response to the loss of N2B (Radke et al., 2007). The deletion of one of the spring elements in the I-band necessitates increased stretch of the other two spring elements to compensate for the loss of extension that the deleted element previously contributed.

Post-translational modifications represent a short-term mechanism for altering the force-extension dynamics of titin's extensible I-band. Kinase phosphorylation of various I-band elements is one of the most extensively studied aspects of myocardial modulation. Protein kinase A, C, and G (PKA, PKC, and PKG) have all been shown to phosphorylate either the N2B or PEVK element of titin's I-band (Yamasaki et al., 2002; Kruger et al., 2009; Hidalgo et al., 2009). The N2B and PEVK domains are the primary sources of titin-based passive force development (Granzier and Labeit, 2007), and kinase phosphorylation alters myocardial passive stiffness by changing 
the force-extension relationships of these domains. Phosphorylation of the N2B element accompanied PKA-dependent passive tension reduction in isolated myocytes (Yamasaki et al., 2002), and site-directed mutagenesis showed that N2B is a PKG substrate that, when phosphorylated, reduces N2B resistance to stretch (Kruger et al., 2009). Furthermore, hypophosphorylation of titin has been associated with high levels of passive tension in patients with heart failure (HF) (Borbely et al., 2009).

Recently, it was shown that PKC $\alpha$, a key member of the PKC serine/threonine kinase family, phosphorylates titin (Hidalgo et al., 2009). In vitro kinase assays determined that titin's extensible PEVK domain is a PKC $\alpha$ substrate, and the combination of mass spectrometry and site-directed mutagenesis pinpointed two PEVK residues as the primary PKC $\alpha$ phosphorylation sites: serine 26 and serine 170 . Muscle mechanics experiments from the same study found that PKC $\alpha$ treatment on skinned left ventricle (LV) myocardium increased passive tension. In order to measure how the individual serine residues affect passive tension development, both pre- and post-PKC $\alpha$ phosphorylation, we performed single-molecule force spectroscopy on WT PEVK and three mutant PEVK: serine 26 mutated to alanine (S26A), serine 170 mutated to alanine $(\mathrm{S} 170 \mathrm{~A})$, and a S170A/S26A double mutant. The purpose of this study was to determine the relative contributions of both PKC $\alpha$ phosphorylation sites to changes in PEVK extensibility.

\section{Materials and methods}

The recombinant PEVK (N2B cardiac isoform) molecules were expressed in Escherichia coli as previously described (Watanabe et al., 2002) with site-directed mutagenesis as in Labeit et al. (2003). We constructed three PEVK mutants (two single mutants - S26A and S170A - and one double mutant - S170A/S26A) in addition to WT PEVK molecules. The purified recombinant PEVK fragments were phosphorylated via an in vitro kinase assay as described (Hidalgo et al., 2009), with minor modifications. Briefly, the purified fragments $(\sim 0.3 \mu \mathrm{g} / \mu \mathrm{l})$ were incubated with recombinant PKC $\alpha$ human (Enzo Life Sciences) $(0.066 \mathrm{U} / \mu \mathrm{I})$ in activating solution (in mM: BES $6.7 \mathrm{pH} 7.0, \mathrm{CaCO}_{3}$-EGTA 1.7, $\mathrm{MgCl}_{2} 2.5, \mathrm{Na}_{2}$-ATP 11.0, DTT 0.25 , K-propionate 7.6 , creatine phosphate $2.5, \mathrm{NaCl}$ 38.3, glycerol 5.3\%), lipid activator (Upstate) (phosphatidylserine $0.17 \mathrm{mg} / \mathrm{ml}$, diacylglycerol $0.02 \mathrm{mg} / \mathrm{ml}$, Triton X-100 0.05\%), phosphatase inhibitors $\left(\mathrm{NaF} 8.3 \mathrm{mM}, \mathrm{Na}_{3} \mathrm{VO}_{4} 1.7 \mathrm{mM}\right)$, and protease inhibitors (leupeptin $20 \mu \mathrm{g} / \mathrm{ml}$, E-64 $10 \mu \mathrm{M}$, and PMSF $0.2 \mathrm{mM}$ ). The samples were incubated for $6 \mathrm{~h}$ at $30^{\circ} \mathrm{C}$, aliquoted, quick frozen in liquid nitrogen, and stored at $-80^{\circ} \mathrm{C}$. Phosphorylation levels were determined by a radioactive kinase assay using $\left[\gamma-{ }^{32} \mathrm{P}\right]$ ATP and autoradiography: see Results in Hidalgo et al. (2009). Measured phosphorylation levels varied depending on incubation and relative kinase concentration. Incubation time and PKC $\alpha$ concentration were selected such that maximum phosphorylation levels were realized. This systematic approach gave us confidence that the vast majority of PEVK were phosphorylated using this optimized protocol.

PEVK molecules, flanked by their native Ig domains (Ig27 and Ig84), were stretched at a constant speed of $1000 \mathrm{~nm} / \mathrm{s}$ using an atomic force microscope (AFM) (MFP3D; Asylum Research, Santa Barbara, CA) as previously described (Zhu et al., 2009). Briefly, $10 \mu \mathrm{g} / \mathrm{ml}$ of protein in assay buffer (AB; $25 \mathrm{mM}$ BES, $2.5 \mathrm{mM}$ EGTA, $1.5 \mathrm{mM} \mathrm{MgCl}, 1.25 \mathrm{mM}$ NaATP, $165 \mathrm{mM} \mathrm{KCl}, 5 \mathrm{mM}$ DTT, pH 7.0) was spotted on a cleaned glass microscope slide. To allow for maximal adsorption, the spotted slides were incubated overnight at $4{ }^{\circ} \mathrm{C}$. Unbound molecules were removed from the slide through multiple washes with $200 \mu \mathrm{l} \mathrm{AB}$. Surface protein density was kept low to minimize nonspecific protein-protein interactions and the probability of multiple molecules attaching to the AFM tip. Ad- sorbed Ig27-PEVK-Ig84 molecules were stretched in AB by contacting the non-conductive silicon nitride cantilever tip (MLCT; Veeco Probes) with the protein-coated surface and retracting the cantilever at $1000 \mathrm{~nm} / \mathrm{s}$. Tip extension-retraction cycles tethered our Ig-PEVK-Ig fragment about $1 \%$ of the time. When a molecule attached to the cantilever tip, a force versus displacement curve was generated as the tip was retracted from the surface. The displacement of the cantilever base was determined via an integrated linear voltage differential transformer. To determine the longitudinal force exerted on the molecule as the tip is pulled away from the surface, Hooke's Law $F=-k x$ was used, where $F(x)$ is the force needed to extend the molecule a given distance $x$ and $k$ is the spring constant of the cantilever. Cantilever stiffness was established by measuring its mean thermally driven vertical displacement $(x)$ and then applying the equipartition theorem: $k<x^{2}>=k_{\mathrm{B}} T$, where $k_{\mathrm{B}}$ is Boltzmann's constant and $T$ is absolute temperature. Typical cantilever stiffness was $\sim 25-30 \mathrm{pN} / \mathrm{nm}$, and the root-mean-square (rms) force noise was $\sim 15 \mathrm{pN}$ for an unloaded cantilever (no tethered molecules). This rms force noise value reveals the fluctuations in our force readings due to thermal energy (as well as system imperfections). To accurately measure the force-extension relationship of PEVK, the end-to-end length $(z)$ of the tethered molecule (the distance between the glass surface and the cantilever tip) was calculated by correcting the cantilever base displacement $(\xi)$ for cantilever bending: $z=\xi-F / k$.

We analyzed the force-extension traces generated by stretching the PEVK molecule by fitting the force versus displacement data with the wormlike chain (WLC) equation (Bustamante et al., 1994):

\section{$F \cdot \mathrm{PL} / k_{\mathrm{B}} T=z / \mathrm{CL}+(1 / 4)(1-z / \mathrm{CL})^{-2}-1 / 4$}

$F$ is the force needed to extend the molecule $(\mathrm{pN}), \mathrm{PL}$ is the persistence length of the molecule $(\mathrm{nm})$ (a quantification of the molecule's bending rigidity), $z$ is the end-to-end length of the molecule $(\mathrm{nm}), \mathrm{CL}$ is the molecule's contour length $(\mathrm{nm}), k_{\mathrm{B}}$ is Boltzmann's constant, and $T$ is absolute temperature. Note that both PL and $\mathrm{CL}$ are inversely proportional to force. During repetitive extensionretraction cycles, if the cantilever tip tethers a molecule when it contacts the protein-coated surface, a force-extension curve is generated as the tip pulls away from the surface. Because the molecular end-to-end distance is constrained by the vertical displacement of the cantilever tip, the molecule is stretched during tip retraction (Fig. 1B). As the tip retracts, the molecule resists extension. This restoring force is entropic in nature and is described by the WLC equation. As the molecule is stretched, the entropic force increases and the cantilever tip is displaced farther from equilibrium. On our force-extension traces, this relationship is seen as a slow increase in force at short extensions that nonlinearly increases as the cantilever tip is pulled farther away from the surface (Fig. 1C). When sufficient force develops, the less stable Ig domain undergoes an abrupt unfolding transition that lengthens the entire molecule; this welldefined unfolding event results in an unfolding force peak. This process is repeated until the second Ig domain unfolds and again when the molecule breaks away from either the surface or tip. The unfolding of an Ig domain should increase the $\mathrm{CL}$ of the molecule from $\sim 4$ to $\sim 26 \mathrm{~nm}$ (folded $\mathrm{Ig} \sim 4 \mathrm{~nm}$, fully extended $\mathrm{Ig}=68$ residues $\times 0.38 \mathrm{~nm} /$ res $\sim 26 \mathrm{~nm}$ ). This sudden increase in CL results in a steep drop in force, as evident in the force-extension curves. We analyzed traces with three regularly spaced force peaks, which ensured that the PEVK domain was fully extended, and fit the trace leading up to the first peak with the WLC equation to determine the $\mathrm{PL}$ and $\mathrm{CL}$ of PEVK.

We also fit the traces leading up to the second and third force peaks to determine the CL increase due to the unfolding of the Ig domains. By fitting all three force peaks and comparing the CL differences between each peak we can determine if the trace was 

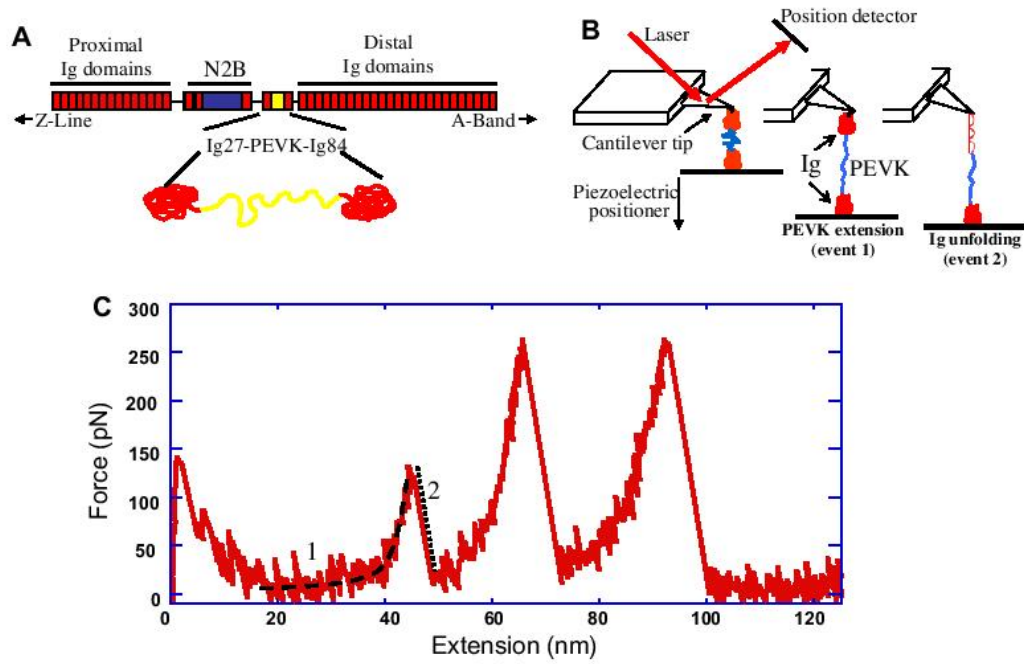

Fig. 1. Single molecule force spectroscopy of PEVK. (A) The I-band of the cardiac titin N2B isoform. Single-molecule force spectroscopy was performed on recombinant protein fragments containing the PEVK domain and flanking Ig domains. (B) Schematic of the AFM experiment. When the cantilever tip tethers our recombinant protein, force develops in the molecule (which bends the tip) as the tip retracts from the surface. Initially, the PEVK element extends (event 1), which generates the force trace leading up to the first force peak. When sufficient force develops, an Ig domain unfolds (event 2), and a distinct force peak develops. This unfolding event increases the $\mathrm{CL}$ of the molecule, which reduces the force in the system immediately after Ig domain unfolding. The second force peak is due to unfolding of the other lg domain, and the third force peak is due to molecular displacement from either the tip or slide. (C) We fit the trace leading up to the first force peak with the WLC equation to determine PEVK resistance to stretch. The force peak at low extensions $(<25 \mathrm{~nm})$ is due to the adhesive force between the cantilever tip and slide surface and is excluded from our WLC fits.

generated by a single Ig-PEVK-Ig molecule tethered between the glass surface and cantilever tip. If a trace showed only three force peaks with peak spacing consistent with the $\mathrm{CL}$ increase subsequent to a single Ig domain unfolding event, we had high confidence that the trace was generated by a single tethered molecule. If the tip had tethered more than one molecule, we would see more than three force peaks. In addition, these force peaks would show unequal spacing due to the independence of Ig domain unfolding between different tethered molecules. The effect of changes in PEVK PL after point mutations and $\mathrm{PKC} \alpha$ phosphorylation at the sarcomere level were calculated with a serially-linked inverted WLC model (Granzier and Labeit, 2005; Moroz and Nelson, 1997).

\subsection{Statistics}

Data are presented as mean \pm SE. Significance was determined using two-way ANOVA, with mutation and PKC $\alpha$ as factors. Post hoc comparisons were made using Tukey HSD. Probability values $<0.05$ were taken as significant.

\section{Results}

It was recently found that $\mathrm{PKC} \alpha$ phosphorylates cardiac N2B PEVK at two highly-conserved serine residues. In order to deduce the individual contributions of the two residues to the dynamics of passive tension in the sarcomere, we probed four PEVK constructs - wildtype, serine 170 mutated to alanine ( $\mathrm{S} 170 \mathrm{~A})$, serine 26 mutated to alanine (S26A), and a S170A/S26A double mutant - using single-molecule force spectroscopy. The WT and all three mutant PEVK are flanked by an Ig domain on either side, which creates a characteristic saw-tooth pattern when the molecule is stretched and unfolded (Fig. 1C). The force peak below $25 \mathrm{~nm}$ extension is due to adhesion between the cantilever tip and slide surface and is excluded from our WLC fits. The force-extension trace up to the first unfolding peak (event 1 in Fig. 1B/C) describes the extension of PEVK, and by fitting this trace with the WLC equation, we quantified the two parameters that determine molecular force-extension dynamics: PL and CL. Fig. 2 shows typical results for all four PEVK constructs in the absence of PKC $\alpha$; each trace shows a similar characteristic "fingerprint" representing two Ig domain unfolding events followed by displacement of the tethered molecule from one or both of its anchoring points.

To gain insight regarding PEVK's structural mechanics, we examined the effects of point mutations on the PL of PEVK prior to $\mathrm{PKC} \alpha$ treatment. By fitting the force trace leading up to the first force peak with the WLC equation, we can extract the PL of PEVK. Fig. 3 shows that all three mutant PEVK have significantly decreased PL values compared to WT (data summary in Table 1). There is no significant difference among mutants. This result suggests that both phosphorylation sites on the PEVK domain are important for maintaining native structure, and that disrupting this structure increases titin-based passive tension. The WT PEVK PL value presented here is slightly lower than our previous study $(\sim 1.0 \mathrm{~nm}$ vs. $\sim 1.2 \mathrm{~nm}$ ) (Hidalgo et al., 2009), but this difference, as well as the PL difference post-PKC $\alpha$, is not significant. However, $\mathrm{PKC} \alpha$ phosphorylation does show a greater effect in our previous study, although the determinant of this difference is currently unclear. The structure of PEVK is not known (it was initially believed to be a random coil), but it has more recently been suggested that PEVK may contain polyproline II helix motifs (Ma et al., 2001; Gutierrez-Cruz et al., 2001), although the high concentration of proline residues and charge clusters is believed to likely hinder the formation of stable structures (Labeit and Kolmerer, 1995). The data presented here supports the idea that PEVK is not simply a random coil: a point mutation would not change the PL of a random coil to a measurable degree. Mutation of serine 26 decreased PEVK PL by $47 \%$, while mutation of serine 170 decreased PEVK PL by $42 \%$. The reduction in PL following the double mutation (48\%) is comparable to each of the single mutants. This somewhat surprising re- 

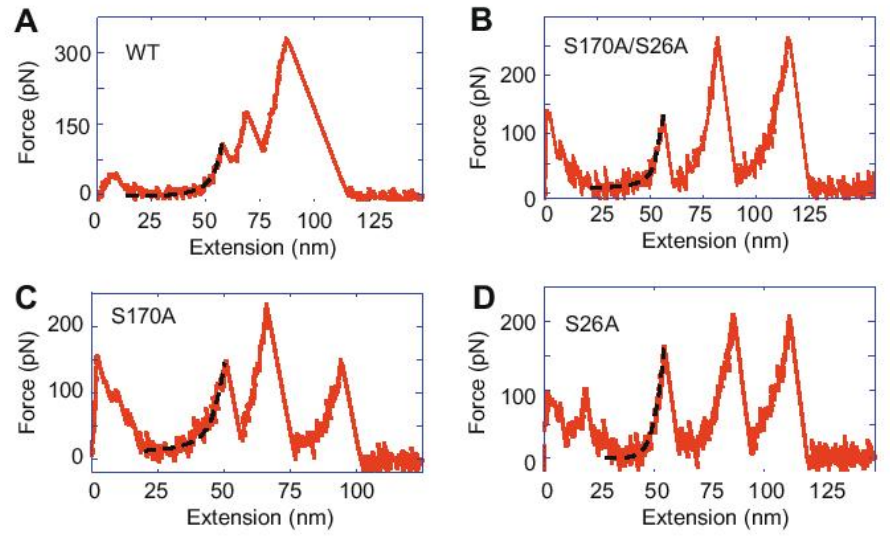

Fig. 2. AFM force-extension curves. Sample force-extension curves for all four PEVK fragments pre-PKC $\alpha$ phosphorylation: (A) WT; (B) S170A/S26A; (C) S170A; (D) S26A. The trace leading up to the first force peak is fit with the WLC equation to extract the PL and CL of PEVK. The force peaks at short extension are due to adhesion between slide surface and cantilever tip, and are excluded from our fits. Note that all peaks have the three-peak "fingerprint" characteristic of an extension-retraction cycle stretching only one Ig-PEVK-Ig molecule.

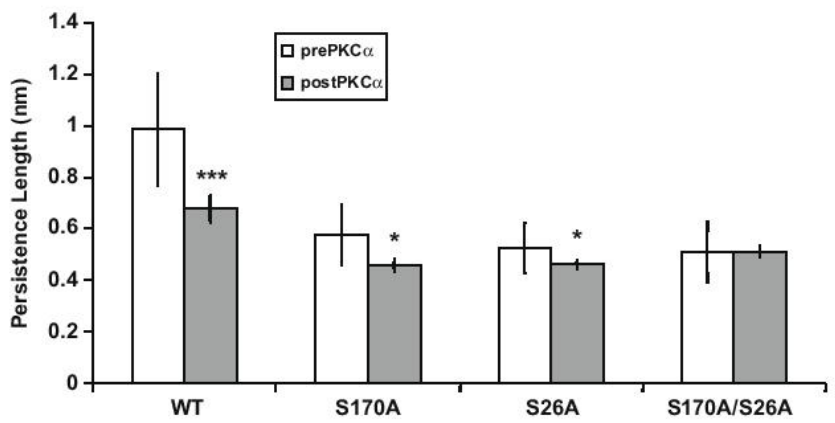

Fig. 3. PEVK PL measurements. Average PL for WT, S170A, S26A, and S170A/S26A fragments, pre- and post-PKC $\alpha$ phosphorylation. Each mutated PEVK shows a significant decrease in PL compared to WT, athough there is no significance a and S170 are the only two residues that influence PEVK mechanics following PKC $\alpha$ phosphorylation. This also supports the notion that there are only two PKC $\alpha$ substrates in the PEVK region. ( $\bullet$ significant vs. pre-PKC $\alpha$ WT; " significant vs. pre-PKC $\alpha$ for given PEVK group; three symbols, $p<0.001$; one symbol, $p<0.05$ ).

Table 1

The effect of point mutations and PKC $\alpha$ phosphorylation on the PL of PEVK.

\begin{tabular}{llll}
\hline & Pre-PKC $\alpha$ PL $(\mathrm{nm})$ & Post-PKC $\alpha$ PL $(\mathrm{nm})$ & Percent change \\
\hline WT & $0.986 \pm .0649$ & $0.676 \pm .0557^{* * *}$ & -31.4 \\
S170A & $0.575 \pm .0420^{* * \bullet}$ & $0.459 \pm .0281^{*}$ & -20.17 \\
S26A & $0.525 \pm .0215^{* * *}$ & $0.463 \pm .0189^{*}$ & -11.81 \\
S170A/S26A & $0.510 \pm .0368^{* * *}$ & $0.513 \pm .0267$ & +0.59 \\
\hline
\end{tabular}

"Significant compared to pre-PKC $\alpha$; ${ }^{\bullet}$ significant compared to pre-PKC $\alpha$ WT. One symbol, $p<0.05$; three symbols, $p<0.001$.

sult suggests that, while each single mutation disrupts the local structure of the PEVK to reduce overall PL, concurrent mutation of both serine residues allows new interactions to develop among regions of the PEVK that were altered by a single mutation. The specific peptide-peptide interactions unique to PEVK fragments with both point mutations - with each mutation affecting the structure associated with that particular residue - require further study.

The extensibility of PEVK was also studied after PKC $\alpha$ phosphorylation. Fig. 3 shows that phosphorylation decreased the PL of all
PEVK constructs except the S170A/S26A mutant. The most significant decrease was observed in WT PEVK, which demonstrated a $31 \%$ drop in bending rigidity. Phosphorylation of serine 26 resulted in a $20 \%$ PL decrease, and phosphorylation of serine 170 induced a $12 \%$ decrease (Table 1 ). PKC $\alpha$ had no effect on the double mutant, which supports the hypothesis that cardiac N2B PEVK has only two critical phosphorylation sites. It is interesting to note that the PL reduction of the individual serine residues combine to account for the entire reduction of PL in WT PEVK. It has been shown that serine 26 is the primary PKC $\alpha$ substrate (Hidalgo et al., 2009), and the PL data presented herein trends in support of that observation.

We also investigated the effects of point mutations and PKC $\alpha$ phosphorylation on the CL of PEVK. In the absence of PKC $\alpha$, there is no significant difference between any PEVK populations, and the average PEVK CL is $59.6 \pm 1.5 \mathrm{~nm}$. If PEVK was a random-coil structure, one would expect that the CL would be $\sim 71 \mathrm{~nm}$ ((188 aa) $*(0.38 \mathrm{~nm} / \mathrm{aa}))$. We previously reported WT PEVK CL to be $\sim 71 \mathrm{~nm}$ (Hidalgo et al., 2009), but upon removal of outliers (more than 2 SD away from mean), the previous data and current data are not significantly different. The shorter than expected CL shown in this study therefore suggests that PEVK contains structural motifs, 
formed from amino acid interactions, which effectively shorten PEVK's CL compared to a random-coil polypeptide. Although there is no significant CL differences between PEVK groups pre-PKC $\alpha$ there is significant change within a group after PKC $\alpha$ phosphorylation. Fig. 4 shows that the PEVK CL, which is determined by fitting the force-extension trace up to the first unfolding peak with the WLC equation, is significantly reduced in both the WT and S170A mutants. WT CL was reduced from $62.5 \pm 3.5$ to $54.6 \pm 1.5 \mathrm{~nm}$ $(p<0.05)$ and $\mathrm{S} 170 \mathrm{~A}$ CL was reduced from $63.3 \pm 2.9$ to $53.2 \pm 1.7 \mathrm{~nm}(p<0.01)$. This small CL reduction following PKC $\alpha$ is not present in previous work (Hidalgo et al., 2009); a possible explanation is that the previous study was more restrictive in analyzing force-extension traces that showed contour lengths less than expected.

We also determined whether or not the point mutations or PKC $\alpha$ activity altered Ig dynamics. To investigate this, we analyzed the difference in CL between successive force peaks in our force-extension curves. The first two force peaks represent the transition of an Ig domain from a compact, globular structure to an unfolded, polypeptide chain. Assuming that the PEVK domain is fully extended (except for the PEVK structures with high stability) before the first Ig domain unfolds, the increase in $\mathrm{CL}$ between consecutive force peaks is solely attributed to an Ig unfolding event. Fig. 5 plots CL versus peak number for all PEVK fragments, both pre- and post-PKC $\alpha$ phosphorylation. The slope of the line fit to $\mathrm{CL}$ vs. peak number represents the average CL gain per unfolding event. The average $\mathrm{CL}$ gain pre-PKC $\alpha$ treatment for $\mathrm{WT}$ $\mathrm{S} 170 \mathrm{~A}, \mathrm{~S} 26 \mathrm{~A}$, and $\mathrm{S} 170 \mathrm{~A} / \mathrm{S} 26 \mathrm{~A}$ were $23.4,23.9,24.4$, and $23.5 \mathrm{~nm}$, respectively, with no significance between PEVK groups. Following PKC $\alpha$ treatment, the average CL gain for WT, S170A, $\mathrm{S} 26 \mathrm{~A}$, and S170A/S26A were 24.3, 26.9, 28.2, and $25.3 \mathrm{~nm}$, respectively, again with no significance between groups. Average CL gain slightly increases following PKC $\alpha$ phosphorylation, but none of these increases are significant within a group, e.g., pre-PKC $\alpha$ WT vs. post-PKC $\alpha$ WT.

The unfolding force (UF) of the Ig peaks was also analyzed. There was no significant change in UF following PKC $\alpha$ phosphorylation of PEVK, an effect which is expected because PKC $\alpha$ presumably does not phosphorylate the Ig domains of cardiac titin's Iband. The mean UF of the Ig domains is presented in Table 2. Although the UF of peak 1 is lower in WT PEVK compared to the mutants, there is only significant difference between WT and S26A prior to PKC $\alpha$ phosphorylation. For a comparison with UF of other Ig domains, see below.

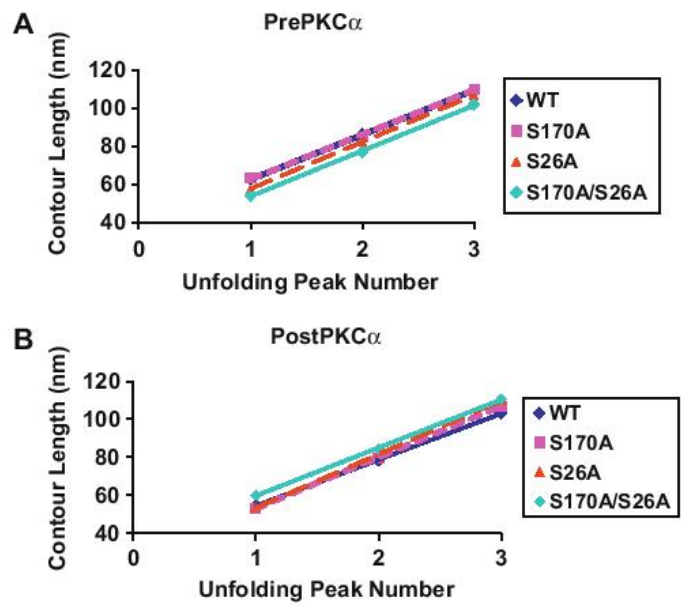

Fig. 5. CL gain following $\lg$ unfolding. To determine the average $\mathrm{CL}$ gain following $\mathrm{I}$ domain unfolding we plot mean $\mathrm{CL}$ vs. force peak number. After both Ig domains unfold, force still develops as the cantilever is retracted because the molecule is still anchored to the slide surface and cantilever tip. The third force peak in our forceextension traces is due to the molecule de-adsorbing from either or both of its anchoring contact points, which is a stochastic process. Therefore, a well-defined force peak may not always develop due to premature displacement of the molecule from slide or tip. To accurately measure the $\mathrm{CL}$ gain between peaks 2 and 3 , we disregarded force-extension curves that did not contain a third force peak that could be well-fit with the WIC equation. (A) Pre-PKCa, the mean CL gain th unfolding for WT, S170A, S26A, and S170A/S26A is $23.4,23.9,24.6$, and $23.5 \mathrm{~nm}$ respectively, with no significance between groups. (B) Post-PKC $\alpha$, the mean $C L$ gain for WT, S170A, S26A, and S170A/S26A is $24.3,26.9,28.2$, and 25.3 , respectively, again with no significance between groups. In addition, there is no significance preand post-PKC $\alpha$ for a given group.

Table 2

Ig domain mean unfolding force $(\mathrm{pN})$

\begin{tabular}{lllcl}
\hline & $\mathrm{WT}-/-$ & $\mathrm{S} 170 \mathrm{~A}-/-$ & $\mathrm{S} 26 \mathrm{~A}-/-$ & $\mathrm{S} 170 \mathrm{~A} / \mathrm{S} 26 \mathrm{~A}-/-$ \\
\hline Peak 1 & $116 \pm 8$ & $156 \pm 11$ & $163^{\circ} \pm 9$ & $156 \pm 14$ \\
Peak 2 & $185 \pm 27$ & $178 \pm 16$ & $185 \pm 17$ & $185 \pm 32$ \\
& $\mathrm{WT}+/+$ & $\mathrm{S} 170 \mathrm{~A}+/+$ & $\mathrm{S} 26 \mathrm{~A}+\left.\right|_{+}$ & $\mathrm{S} 170 \mathrm{~A} / \mathrm{S} 26 \mathrm{~A}+/+$ \\
\cline { 2 - 5 } Peak 1 & $132 \pm 10$ & $166 \pm 15$ & $165 \pm 12$ & $155 \pm 12$ \\
Peak 2 & $158 \pm 13$ & $166 \pm 26$ & $184 \pm 23$ & $187 \pm 20$ \\
\hline
\end{tabular}

Significant compared to WT $-1-, p<0.05$.

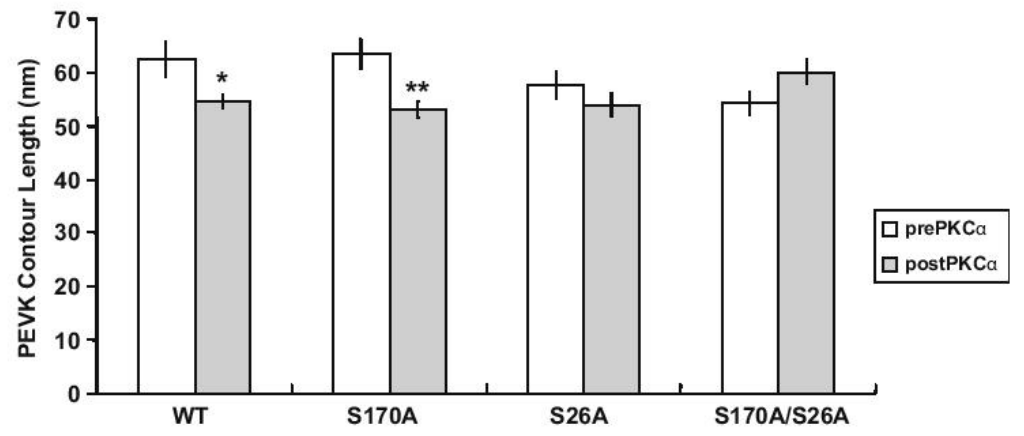

Fig. 4. PEVK CL measurements. PEVK CL is determined from fitting the trace up to the first force peak with the WLC equation. Prior to PKC $\alpha$, there is no significant difference between PEVK fragments. After phosphorylation, WT and S170A PEVK exhibit significant decrease in CL. The addition of $\mathrm{PO}_{4}^{2-}$ to $\mathrm{S} 26$ and $\mathrm{S} 170$ likely reduces $\mathrm{CL}$ via attractive interactions between phosphoserine and positively-charged residues/PEVK repeat motifs. A mild competitive effect from phosphorylated S170 may explain why a slightly larger CL decrease is measured in S170A compared to WT. ("Significant between pre- and post-PKC $\alpha$ sets of a given PEVK group; two symbols, $p<0.01 ;$ one symbol, $p<0.05$. There are no significant differences due to mutations alone). 


\section{Discussion}

Diastolic pressure results from the development of passive force, which is largely determined intracellularly by titin's extensible I-band region and extracellularly by collagen networks. Recent work reveals that both long-term and short-term pathways affect the passive tension dynamics in the myocardium, which result in a variety of similar and disparate effects. The major long-term pathway for modulating myocardial stiffness is titin isoform splicing, a process that occurs on the time frame of weeks (Wu et al., 2002). Numerous direct short-term responses also influence the passive force response to sarcomere stretch, notably the regulation of various protein kinases via upstream adrenergic pathways. PKA and PKG phosphorylation of the elastic N2B element of titin have been associated with passive tension reduction (Yamasaki et al., 2002; Kruger et al., 2009), and our group recently showed that PKC phosphorylates the cardiac titin N2B isoform on two serine residues located within the 188-residue PEVK element, S26 and S170 (Hidalgo et al., 2009). Unlike PKA/PKG phosphorylation of titin's I-band, PKC phosphorylation corresponded with an increase in passive tension. PKC, which is activated by $\alpha_{1}$-adrenergic pathways, has been implicated in various cardiovascular diseases, including ischemic heart disease, cardiac hypertrophy, hypertension, and atherosclerosis (for a recent review see Murphy and Frishman (2005)). It has been shown that PKC $\alpha$, the predominant isoform expressed in mouse, human, and rabbit heart (Hambleton et al., 2006; Pass et al., 2001; Ping et al., 1997), is upregulated in patients with dilated cardiomyopathy and ischemic cardiomyopathy (Bowling et al., 1999), and in rats with end-stage HF (Belin et al., 2007). Mice lacking the gene for PKC $\alpha$ show enhanced cardiac contractility and reduced susceptibility to $\mathrm{HF}$ (Liu et al., 2009), which suggests that PKC inhibitors could be viable candidates for HF treatment.

In order to determine the relative contributions of each phosphorylated serine residue to the measured increase in passive tension following $\mathrm{PKC} \alpha$ treatment, we generated four recombinant PEVK fragments: WT, S26A, S170A, and S170A/S26A. Using single-molecule force spectroscopy, we investigated the effect that point mutations have on the force-extension relationship of PEVK, both pre- and post-PKC $\alpha$ treatment. Because the force-extension response of the elastic spring elements of titin's I-band can be quantified using the WLC equation (Granzier and Labeit, 2007; Watanabe et al., 2002; Zhu et al., 2009; Li et al., 2001; Kellermayer et al., 2001, 1997; Rief et al., 1997), we were able to fit our singlemolecule force spectroscopy data with the WLC equation to extract the relevant physical parameters (PL and CL) of all PEVK fragments (both $+/-$ PKC $\alpha$ ). From these parameters, we are able to model the physiological effect that individual residues have on the sarcomeric resistance to stretch.

On the molecular level, a change in CL or PL is associated with structural transitions or changes in the energy landscape. For example, the formation or destruction of stable secondary (and tertiary) structures may change the effective length of a molecule, and the presence of energetic potentials, e.g., electrostatic, may change the effective stiffness of a molecule (Baumann et al., 1997; Forbes et al., 2005). Recognition of the dynamics of physical changes in a spring-like molecule, such as PEVK, gives insight into that molecule's structural composition. The complete sequence of human cardiac titin shows that the PEVK sequence is primarily composed of $\sim 28$-residue repeats and polyE motifs (Bang et al., 2001). In cardiac N2B PEVK, all the negatively-charged polyE motifs and 55 of the 60 (as found in soleus titin) PEVK repeats are spliced out (Greaser, 2001). The PEVK structure is not well-defined, but the existence of structural motifs has been proposed. Although high proline concentration precludes the formation of sizeable $\alpha$-helices and $\beta$-sheet structures (Williamson, 1994; MacArthur and Thorn- ton, 1991), NMR and circular dichroism (CD) experiments suggest that polyproline helix-coil motifs are fundamental structural features of PEVK (Ma et al., 2001; Gutierrez-Cruz et al., 2001). In addition, the PEVK sequence, especially around S26 and S170, is highly conserved in a wide variety of species, which also suggests that PEVK is structured, at least in part (Hidalgo et al., 2009). If PEVK was simply a molecular random coil, its elastic spring properties would not be significantly altered by sequence drift and would not be evolutionarily conserved.

We found that WT PEVK has $\mathrm{PL} \sim 1 \mathrm{~nm}$ in the absence of $\mathrm{PKC} \alpha$, which is consistent with published values (Watanabe et al., 2002; Li et al., 2001; Linke et al., 2002). Serine-to-alanine mutations significantly reduced PL in both single mutants and the double mutant (Fig. 3); there was no significant difference in PL among the three mutant populations. The significant decrease of PEVK PL following both serine-to-alanine point mutations suggests that both S26 and S170 are important in maintaining structural integrity. Physically, a reduction in PL of an amino acid polypeptide can be attributed to the dissolution of stabilizing interactions, e.g., hydrogen bonds, between domains, which increases the translational and rotational degrees of freedom of the peptide sequences that were previously constrained by the stabilizing interaction. The "release" of these degrees of freedom increases the number of configurations that the molecule can occupy. This enlargement of phasespace volume that the molecule can populate increases the relative entropy associated with the molecule's configuration at a given end-to-end length. An increase in molecular end-to-end length reduces the entropy of the system and is therefore statistically improbable. This resistance to a lower entropic system, or entropic force, favors a compact protein conformation, and is the restoring force that opposes molecular extension in AFM experiments. The WLC equation shows that the force needed to extend an entropic spring is inversely proportional to the molecule's PL, implying that a measured decrease in PL corresponds to an increase in molecular entropy. Therefore, it is proposed that the S26A and S170A mutations disrupt the stabilizing interactions involving the native serine residues, which increases the conformational entropy of PEVK and results in a lower effective PL.

We focused our investigation on the effect that PKC $\alpha$ phosphorylation has on the sarcomeric force-extension relationship by quantifying the phosphorylation effect at the single molecule level. Comparing the PL differences post-PKC $\alpha$ treatment suggests that S26 plays a bigger role than S170 in the modulation of myocardial stiffness. The $31 \%$ reduction in PL of WT PEVK post-PKC $\alpha$ is well accounted for by the $20 \%$ and $12 \%$ PL reduction measured in S170A and S26A, respectively. The larger effect measured in the S170A mutant could be due to several factors. One factor is that S26 has been shown to be more readily phosphorylated by $\mathrm{PKC} \alpha$ compared to S170 (Hidalgo et al., 2009). Another factor could be that S26 is more actively involved in determining the structure of PEVK compared to $\mathrm{S} 170$, and that phosphorylation disturbs these interactions. However, the PL values of S26A and S170A pre-PKC $\alpha$ are not significantly different, which is not consistent with the latter explanation. It is more likely that PKC $\alpha$ phosphorylation has a larger effect on the PL of S170A because S26 is the primary site of $\mathrm{PKC} \alpha$ phosphorylation.

PKC $\alpha$ phosphorylation also reduced CL in WT and S170A PEVK. Prior to PKC $\alpha$, PEVK CL is $\sim 60 \mathrm{~nm}$, which is less than expected for an unfolded 188-residue polypeptide. However, the substantial reduction in PL following point mutations and PKC $\alpha$ phosphorylation necessarily implies that PEVK contains structured domains, and our CL measurements are consistent with this. The small CL reduction post-PKC $\alpha$ can be explained by electrostatic considerations. The addition of a negatively-charged phosphate molecule to the polar side chain of S26 or S170, perhaps more pronounced in S26, effectively changes the serine into a polar residue with a 
negatively charged side chain. At neutral $\mathrm{pH}$, phosphoserine typically carries a -2 net charge (Andrew et al., 2002) due to phosphoric acid's $\mathrm{p} K_{\mathrm{a} 2}$ value of $\sim 6$.1. Cardiac N2B PEVK comprises five $\sim 28$ residue PEVK repeats, which carry a net positive charge and have isoelectric points between 9 and 10 (AFM experiments executed at $\mathrm{pH} 7$ ). In addition, there are 43 combined arginine and lysine residues (which contain positively-charged side chains) in the 188-residue PEVK sequence. The negative charge of phosphoserine may attractively interact with either positively-charged peptides in the PEVK repeats or with specific arginine/lysine residues. This attractive potential between segments of the PEVK that would normally be repulsive (or have no interaction potential) could explain the reduced $\mathrm{CL}$ after $\mathrm{PKC} \alpha$ phosphorylation.

The CL gain between successive force peaks is attributed to the unfolding of an Ig domain. The average CL gain between force peaks for all samples is $\sim 25 \mathrm{~nm}$, which is slightly higher than expected assuming the absence of all secondary structure following Ig unfolding. Ig27 and Ig84 contain 68 and 67 residues, respectively, as determined by Simple Modular Architecture Research Tool (SMART) software (Letunic et al., 2009; Schultz et al., 1998), and we therefore expect to see a $\sim 22 \mathrm{~nm}$ CL increase following an Ig domain unfolding event ( $\sim 26 \mathrm{~nm}$ for fully unfolded Ig minus the diameter of a folded $\mathrm{Ig}$ domain $(\sim 4 \mathrm{~nm})$ ). The slightly higher than expected $\mathrm{CL}$ gain can be explained by the unfolding of structural motifs in PEVK that were stable up to the first force peak.

We also analyzed the UF of Ig27 and Ig84, and found that PKC $\alpha$ does not affect Ig UF, as expected. An unexplained finding that requires further study is that UF is lower for WT PEVK than for the mutants. However, a comparison with previous results of Ig domains from titin's proximal and distal tandem Ig segments shows that Ig27 and Ig84 are relatively unstable. Considering all PEVK groups (both pre- and post-PKC $\alpha$ phosphorylation), the average UF of peak 1 and peak 2 is $152 \pm 4$ and $178 \pm 7 \mathrm{pN}$, respectively. At the same pulling speed $(1000 \mathrm{~nm} / \mathrm{s})$, it has been shown that the average UF of tandem Ig 1-8 and $\operatorname{Ig} 8-15$ is $\sim 221 \mathrm{pN}$ and $\sim 232 \mathrm{pN}$, respectively (Zhu et al., 2009). At $500 \mathrm{~nm} / \mathrm{s}$ stretch speed, the mean unfolding force of tandem Ig 91-98 domains was reported to be $\sim 230 \mathrm{pN}$ (Watanabe et al., 2002). Closer to the UF measured in this study, AFM experiments that stretched the flexible N2B element of the I-band, along with the three Ig domains (Ig 24-26) that flank the N2B element (see Fig. 1A), showed that the average UF of these flanking Ig domains, from least stable to most stable, are $\sim 147, \sim 177$, and $\sim 191 \mathrm{pN}$. Combined with the UF results of Ig 24-26, our results of Ig27 and Ig84 suggest that the Ig domains that immediately flank the elastic N2B and PEVK regions of cardiac titin's N2B isoform are less mechanically stable than the proximal and distal tandem Ig domains that define the $\mathrm{N}$ and $\mathrm{C}$ termini of the I-band, respectively. SMART software determined that all but one of the Ig-like domains from the Ig 1-15 and Ig 91-98 set contain between 80 and 90 residues and belong to the general Ig class of proteins. However, Ig25, Ig26, Ig27, and Ig84, the four domains that immediately flank N2B and PEVK, respectively, all belong to the $\operatorname{IgC} 2$ family of immunoglobulin and contain between $65-70$ residues. This structural difference between the Ig domains that flank the two dynamic spring elements in the I-band and the tandem Ig domains in titin may help explain the significant differences in UF between the two groups.

We used a serially-linked WLC model (Granzier and Labeit, 2005; Moroz and Nelson, 1997) to determine the effect that PEVK PL and CL changes have on the titin-based tension that develops at SLs greater than slack length. Fig. 6 shows that, following PKC $\alpha$ phosphorylation, passive tension increases, an effect attributed to $\mathrm{PL}$ and $\mathrm{CL}$ reduction following PKC $\alpha$ phosphorylation. Reduced PL is responsible for the majority of force increase at shorter SL, and reduced CL results in higher force increases at longer SLs. The SLdependent force contribution of reduced CL stems from the nonlin-

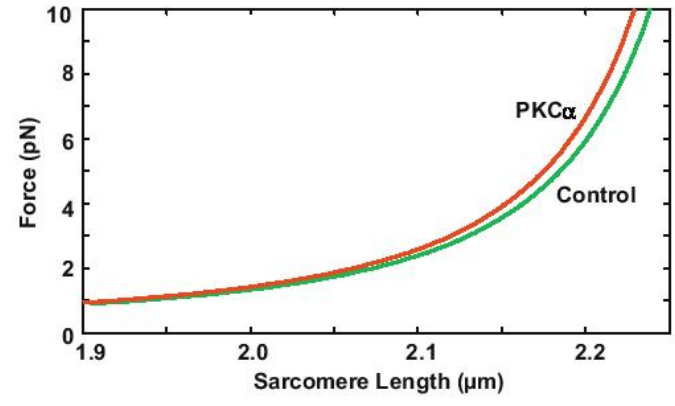

Fig. 6. The effect of $\operatorname{PKC} \alpha$ on titin-based passive tension in the sarcomere. To determine how changes in PEVK PL and CL alter the resistance to stretch of an entire titin molecule, we use an inverted WLC equation to sum the relative extensions of all three I-band elements at a given force. For a single titin molecule (cardiac N2B isoform), $\mathrm{PKC} \alpha$ phosphorylation increases the force needed to extend titin at a given length. At $\mathrm{SL} \sim 2.2 \mu \mathrm{m}$, we calculate that $\mathrm{PKC} \alpha$. phosphorylation increases passive force by $15 \%$.

ear dependence of CL on entropic force, as described by the WLC equation. At a $\mathrm{SL} \sim 2.2 \mu \mathrm{m}$, the post-PKC $\alpha$ force increase $(\sim 15 \%)$ derives equally from PL and CL decreases. In skinned mouse myocardium (which primarily expresses the N2B isoform (Lahmers et al., 2004)), it was shown that PKC $\alpha$ phosphorylation increases titin-based passive tension by $\sim 25 \%$ at a SL of $2.2 \mu \mathrm{m}$. Our data suggests that the majority of this passive tension increase derives from direct phosphorylation of S26 and S170 in the PEVK element. Upon phosphorylation of these residues in cardiac tissue, the PEVK PL and CL decrease, and more external work is needed to increase sarcomere length and expand the heart.

In summary, AFM studies indicate that both PKC $\alpha$ substrates in the extensible I-band of cardiac N2B titin, S26 and S170 of the PEVK element, are important for the mechanical properties of the myocardium. The force-extension dynamics of PEVK are important in determining the development of passive tension in stretched myocardium, and mutation of S26 and S170 significantly changes PEVK response to stretch. PKC $\alpha$ phosphorylation of S26 and S170 al so increases the force needed to extend PEVK in a residue-specific manner, and we propose that this is a novel mechanism for modulating myocardial passive stiffness.

\section{Disclosures}

None.

\section{Acknowledgments}

We thank Carlos Hidalgo for preparation of phosphorylated samples. Brian Anderson received support from NIH training Grant GM084905. Supported by NIH HL062881 to H.G. and by the DFG (La668/13-1) and the NAR Initiative University of Heidelberg to S.L.

\section{References}

Andrew, C.D. et al., 2002. Effect of phosphorylation on alpha-helix stability as a function of position. Biochemistry 41 (6), 1897-1905

Bang, M.L. et al., 2001. The complete gene sequence of titin, expression of an unusual approximately $700-\mathrm{kDa}$ titin isoform, and its interaction with obscurin identify a novel Z-line to I-band linking system. Circ. Res. 89 (11), 1065-1072. Baumann, C.G. et al., 1997. lonic effects on the elasticity of single DNA molecules. Proc. Natl. Acad. Sci. USA 94 (12), 6185-6190.

Belin, R.J. et al., 2007. Augmented protein kinase C-alpha-induced myofilament protein phosphorylation contributes to myofilament dysfunction in experimental congestive heart failure. Circ. Res. 101 (2), 195-204. 
Borbely, A. et al., 2009. Hypophosphorylation of the stiff N2B titin isoform raises cardiomyocyte resting tension in failing human myocardium. Circ. Res. 104 (6) 780-786.

Bowling, N. et al., 1999. Increased protein kinase C activity and expression of $\mathrm{Ca}^{2+}$ sensitive isoforms in the failing human heart. Circulation 99 (3), 384-391.

Bustamante, C. et al., 1994. Entropic elasticity of lambda-phage DNA. Science 265 (5178), 1599-1600

Forbes, J.G. et al., 2005. Titin PEVK segment: charge-driven elasticity of the open and flexible polyampholyte. J. Muscle Res. Cell Motil. 26 (6-8), 291-301.

Freiburg, A. et al., 2000. Series of exon-skipping events in the elastic spring region of titin as the structural basis for myofibrillar elastic diversity. Circ. Res. 86 (11) $1114-1121$

Furst, D.O. et al., 1988. The organization of titin filaments in the half-sarcomere revealed by monoclonal antibodies in immunoelectron microscopy: a map of ten nonrepetitive epitopes starting at the $\mathrm{Z}$ line extends close to the $\mathrm{M}$ line. Cell Biol. 106 (5), 1563-1572.

Granzier, H.L., Labeit, S., 2005. Titin and its associated proteins: the thirc myofilament system of the sarcomere. Adv. Protein Chem. 71, 89-119.

Granzier, H., Labeit, S., 2007. Structure-function relations of the giant elastic protein titin in striated and smooth muscle cells. Muscle Nerve 36 (6), 740-755.

Granzier, H.L. et al., 2009. Truncation of titin's elastic PEVK region leads to cardiomyopathy with diastolic dysfunction. Circ. Res. 105 (6), 557-564.

Greaser, M., 2001. Identification of new repeating motifs in titin. Proteins 43 (2), $145-149$.

Gutierrez-Cruz, G., Van Heerden, A.H., Wang, K., 2001. Modular motif, structura folds and affinity profiles of the PEVK segment of human fetal skeletal muscle titin. J. Biol. Chem. 276 (10), 7442-7449.

Hambleton, M. et al., 2006. Pharmacological- and gene therapy-based inhibition of protein kinase Calpha/beta enhances cardiac contractility and attenuates heart failure, Circulation 114 (6), 574-582

Hidalgo, C. et al., 2009. PKC phosphorylation of titin's PEVK element: a novel and conserved pathway for modulating myocardial stiffness. Circ. Res. 105 (7), 631638. 17 p following 638 .

Horowits, R. et al., 1986. A physiological role for titin and nebulin in skeletal muscle. Nature 323 (6084), 160-164.

Kellermayer, M.S. et al., 1997. Folding-unfolding transitions in single titin molecules characterized with laser tweezers. Science 276 (5315), 1112-1116.

ellemar, MS et at. 2001. Mechanical fatigue in repetitively stretched singe molecules of titin. Biophys. J. 80 (2) 852-863.

Kruger, M. et al., 2009. Protein kinase G modulates human myocardial passive stiffness by phosphorylation of the titin springs. Circ. Res. 104 (1), 87-94.

Labeit, S., Kolmerer, B., 1995. Titins: giant proteins in charge of muscle ultrastructure and elasticity. Science $270(5234), 293-296$.
un

Labeit, D. et al., 2003. Calcium-dependent molecular spring elements in the giant protein titin. Proc. Natl. Acad. Sci. USA 100 (23), 13716-13721.

Lahmers, S. et al, 2004. Developmental control of titin isoform expression and passive stiffness in fetal and neonatal myocardium. Circ. Res. 94 (4), 505-513.
Letunic, I., Doerks, T., Bork, P., 2009. SMART 6: recent updates and new developments. Nucleic Acids Res. 37 (Database issue), D229-D232.

Li, H. et al., 2001. Multiple conformations of PEVK proteins detected by singlemolecule techniques. Proc. Natl. Acad. Sci. USA 98 (19), 10682-10686.

Linke, W.A. et al., 2002. PEVK domain of titin: an entropic spring with actin-binding properties. J. Struct. Biol. 137 (1-2), 194-205.

Liu, Q. et al., 2009. Protein kinase C\{alpha\}, but not PKC\{beta\} or PKC\{gamma\}, regulates contractility and heart failure susceptibility: implications for regulates contractility and heart failure susceptibility: implications

Ma, K. Kan, L, Wang K 2001. Polyproline II helix is a key structural motif of the elastic PEVK segment of titin. Biochemistry 40 (12), 3427-3438.

MacArthur, M.W., Thornton, J.M., 1991. Influence of proline residues on protein conformation. J. Mol. Biol. 218 (2), 397-412.

Moroz, J.D., Nelson, P., 1997. Torsional directed walks, entropic elasticity, and DNA twist stiffness. Proc. Natl. Acad. Sci. USA 94 (26), 14418-14422.

Murphy, S., Frishman, W.H., 2005. Protein kinase C in cardiac disease and as a potential therapeutic target. Cardiol. Rev. 13 (1), 3-12.

Pass, J.M. et al., 2001. Enhanced PKC beta II translocation and PKC beta II-RACK interactions in PKC epsilon-induced heart failure: a role for RACK1. Am. J. Physiol. Heart Circ. Physiol. 281 (6), H2500-H2510.

Ping, P. et al., 1997. Ischemic preconditioning induces selective translocation of protein kinase $\mathrm{C}$ isoforms epsilon and eta in the heart of conscious rabbits without subcellular redistribution of total protein kinase $\mathrm{C}$ activity. Circ. Res. 8 (3), 404-414.

Radke, M.H. et al., 2007. Targeted deletion of titin N2B region leads to diastolic dysfunction and cardiac atrophy. Proc. Natl. Acad. Sci. USA 104 (9), 3444-3449.

Rief, M. et al., 1997. Reversible unfolding of individual titin immunoglobulin domains by AFM. Science 276 (5315), 1109-1112.

Schultz, J. et al., 1998. SMART, a simple modular architecture research tool: identification of signaling domains. Proc. Natl. Acad. Sci. USA 95 (11), 5857 5864.

Trombitas, K. et al., 2000. Extensibility of isoforms of cardiac titin: variation in contour length of molecular subsegments provides a basis for cellular passive stiffness diversity. Biophys. J. 79 (6), 3226-3234.

Watanabe, K. et al., 2002. Molecular mechanics of cardiac titin's PEVK and N2B spring elements. J. Biol. Chem. 277 (13), 11549-11558.

Williamson, M.P., 1994. The structure and function of proline-rich regions in proteins. Biochem. J. 297 (Pt. 2), 249-260.

Wu, Y. et al., 2002. Changes in titin isoform expression in pacing-induced cardiac failure give rise to increased passive muscle stiffness. Circulation 106 (11), 1384-1389.

Yamasaki, R. et al., 2002. Protein kinase A phosphorylates titin's cardiac-specific N2B domain and reduces passive tension in rat cardiac myocytes. Circ. Res. 90 (11), 1181-1188

Zhu, Y. et al., 2009. Single molecule force spectroscopy of cardiac titin's N2B element-effects of the molecular chaperone alpha B-crystallin with disease causing mutations. J. Biol. Chem. 284 (20), 13914-13923. 